\title{
Transition asymptotics of Toeplitz determinants and emergence of Fisher- Hartwig representations
}

Article

Accepted Version

Kozlowska, K. and Virtanen, J. (2019) Transition asymptotics of Toeplitz determinants and emergence of Fisher-Hartwig representations. Nonlinearity, 32 (10). pp. 3593-3645. ISSN 1361-6544 doi: https://doi.org/10.1088/1361-6544/ab127a Available at https://centaur.reading.ac.uk/82901/

It is advisable to refer to the publisher's version if you intend to cite from the work. See Guidance on citing.

To link to this article DOI: http://dx.doi.org/10.1088/1361-6544/ab127a

Publisher: IOP Publishing

All outputs in CentAUR are protected by Intellectual Property Rights law, including copyright law. Copyright and IPR is retained by the creators or other copyright holders. Terms and conditions for use of this material are defined in the End User Agreement.

www.reading.ac.uk/centaur 
Central Archive at the University of Reading

Reading's research outputs online 


\title{
TRANSITION ASYMPTOTICS OF TOEPLITZ DETERMINANTS AND EMERGENCE OF FISHER-HARTWIG REPRESENTATIONS
}

\author{
KASIA KOZLOWSKA AND JANI A. VIRTANEN
}

\begin{abstract}
We compute the transition asymptotics (double-scaling limits) of Toeplitz determinants generated by symbols $f_{t}$ possessing Fisher-Hartwig singularities. The symbols $f_{t}$ that we consider depend on a parameter $t$ such that $f_{t}$ has one Fisher-Hartwig singularity when $t>0$ and two Fisher-Hartwig singularities when $t=0$. Unlike in the other studies of the transition asymptotics of Toeplitz determinants, our setting involves the emergence of Fisher-Hartwig representations as $t \rightarrow 0$. We use the Riemann-Hilbert problem for orthogonal polynomials and its connection to Painlevé transcendents to obtain the asymptotics. We apply our results to study a special correlator known as the emptiness formation probability (EFP) for the one-dimensional anisotropic XY spin$1 / 2$ chain in a transverse magnetic field, and describe its transition between different regions in the phase diagram across critical lines.
\end{abstract}

\section{INTRODUCTION}

For a function $f \in L^{1}=L^{1}(\mathbb{T})$ of the unit circle $\mathbb{T}$ and $n \in \mathbb{N}$, the $n \times n$ Toeplitz matrix $T_{n}(f)$ with symbol $f$ is defined by setting

$$
T_{n}(f)=\left(f_{j-k}\right)_{j, k \geq 0}^{n-1}=\left(\begin{array}{ccccc}
f_{0} & f_{-1} & f_{-2} & \cdots & f_{-(n-1)} \\
f_{1} & f_{0} & f_{-1} & \cdots & f_{-(n-2)} \\
f_{2} & f_{1} & f_{0} & \cdots & f_{-(n-3)} \\
\vdots & \vdots & \vdots & \ddots & \vdots \\
f_{n-1} & f_{n-2} & f_{n-3} & \cdots & f_{0}
\end{array}\right),
$$

where $f_{k}=\frac{1}{2 \pi} \int_{0}^{2 \pi} f\left(e^{i \theta}\right) e^{-i k \theta} d \theta$ are the Fourier coefficients of the function $f$. The determinant of $T_{n}(f)$ is denoted by $D_{n}(f)$. Despite their simple definition, matrices of the form $\left(f_{j-k}\right)$ have played an important role in mathematics and its applications since 1910 when Toeplitz used them to illustrate Hilbert's spectral theory.

The study of Toeplitz determinants began in the 1910s, when Pólya conjectured that if $f$ is positive and continuous, then

$$
\lim _{n \rightarrow \infty} \frac{1}{n} \log D_{n}(f)=(\log f)_{0}=V_{0}=\frac{1}{2 \pi} \int_{0}^{2 \pi} \log f\left(e^{i \theta}\right) d \theta
$$

which was proved by Szegö in 1915. For a positive symbol $f$, each eigenvalue $\lambda_{k}^{(n)}$ of the Toeplitz matrix $T_{n}(f)$ is positive and $(2)$ is equivalent to

$$
\lim _{n \rightarrow \infty} \frac{\log \lambda_{1}^{(n)}+\cdots+\log \lambda_{n}^{(n)}}{n}=(\log f)_{0}=V_{0},
$$

2010 Mathematics Subject Classification. Primary 15B05; Secondary 15A15, 82B20.

Key words and phrases. Toeplitz determinants; Fisher-Hartwig singularities; double-scaling limits; transition asymptotics; the XY model; quantum spin chains.

J. Virtanen was supported in part by Engineering and Physical Sciences Research Council (EPSRC) grant EP/M024784/1. K. Kozlowska was supported in part by EPSRC Doctoral Training Grant and University of Reading Doctoral Scholarship. 
where $V(z)=\log f(z)$. Alternatively, the large $n$ limit in (2) can be written as follows

$$
D_{n}(f)=\exp \left\{n V_{0}+o(n)\right\} \quad \text { as } n \rightarrow \infty .
$$

The precise nature of the error term $o(n)$ is important in applications, such as the two-dimensional Ising model, whose magnetization can be expressed as a large $n$ limit of Toeplitz determinants. More than three decades after his first limit theorem, Szegö became aware of Onsager and Kaufman's work on the Ising model, and in 1952 he proved his strong limit theorem (for positive symbols that have a derivative in a Hölder class), which finally provided the error term. Over the years, the result was generalized to complex-valued matrix symbols in Krein algebras (see Section 10.4 of [2]) and to the case in which symbols are measures (see Chapter 6 of [25]). We formulate the result for scalar symbols. If $f$ is continuous, has no zeros, has no winding, $\sum_{n \in \mathbb{Z}}\left|f_{n}\right|<\infty$ and $\sum_{n \in \mathbb{Z}}(|n|+1)\left|f_{n}\right|^{2}<\infty$, then the strong Szegö limit theorem (SSLT) implies that

$$
D_{n}(f)=\exp \left\{n V_{0}+\sum_{k=1}^{\infty} k V_{k} V_{-k}+o(1)\right\} .
$$

For a detailed account of the fascinating history of Toeplitz determinants with citations to the original works, see, e.g., [2, 7, 25].

1.1. Fisher-Hartwig symbols. A number of problems in statistical mechanics require more general symbols than the continuous symbols above. In 1968 Fisher and Hartwig [13] singled out symbols, which may possess zeros, integrable singularities and non-zero winding numbers. These symbols can be written in the form

$$
f(z)=e^{V(z)} z^{\sum_{j=0}^{m} \beta_{j}} \prod_{j=0}^{m}\left|z-z_{j}\right|^{2 \alpha_{j}} g_{z_{j}, \beta_{j}}(z) z_{j}^{-\beta_{j}}, \quad z=e^{i \theta}, \quad \theta \in[0,2 \pi),
$$

for some $m=\mathbb{N} \cup\{0\}$, where each $z_{j}=e^{i \theta_{j}}, 0=\theta_{0}<\theta_{1}<\cdots<\theta_{m}<2 \pi$,

$$
g_{z_{j}, \beta_{j}}(z)= \begin{cases}e^{i \pi \beta_{j}} & \text { if } 0 \leq \arg z<\theta_{j}, \\ e^{-i \pi \beta_{j}} & \text { if } \theta_{j} \leq \arg z<2 \pi\end{cases}
$$

and

$$
\operatorname{Re} \alpha_{j}>-1 / 2, \quad \beta_{j} \in \mathbb{C}, \quad j=0, \ldots, m,
$$

and $V$ is analytic in a neighborhood of the unit circle. Functions of the form (5) are called FisherHartwig symbols. Observe that the condition $\operatorname{Re} \alpha_{j}>-\frac{1}{2}$ implies that $f \in L^{1}$. Clearly, $f$ has a zero at $z_{j}$ if $\operatorname{Re} \alpha>0$; a pole at $z_{j}$ if $\operatorname{Re} \alpha<0$; and a discontinuity of oscillating type at $z_{j}$ if $\operatorname{Re} \alpha=0$ and $\operatorname{Im} \alpha \neq 0$. Also, note that, for $j>0, z^{\beta_{j}} g_{z_{j}, \beta_{j}}(z)$ is continuous at 1 , so the strength of each jump of $f$ at $z_{j}$ is determined by $\beta_{j}$.

The leading order asymptotics of Toeplitz determinants with Fisher-Hartwig symbols were computed by Ehrhardt in his PhD thesis in 1997 (see also [12]). To state his result, we need the following seminorm

$$
\|\| \beta \|\left|=\max _{j, k}\right| \operatorname{Re} \beta_{j}-\operatorname{Re} \beta_{k} \mid,
$$

where $1 \leq j, k \leq m$ if $\alpha_{0}=\beta_{0}=0$, and $0 \leq j, k \leq m$ otherwise. If $m=0$, we set $\|\beta\|=0$. We also need the Wiener-Hopf factorization

$$
e^{V(z)}=b_{+}(z) e^{V_{0}} b_{-}(z), \quad b_{+}(z)=e^{\sum_{k=1}^{\infty} V_{k} z^{k}}, \quad b_{-}(z)=e^{\sum_{k=-\infty}^{-1} V_{k} z^{k}}
$$

where $V_{k}$ denote the Fourier coefficients of the function $V$. 
Theorem 1. Let $f$ be Fisher-Hartwig, $V(z) \in C^{\infty}(\mathbb{T}),\|\beta\|<1, \operatorname{Re} \alpha_{j}>-\frac{1}{2}$ and $\alpha_{j} \pm \beta_{j} \neq$ $-1,-2, \ldots$ for $j, k=0,1, \ldots, m$. Then as $n \rightarrow \infty$,

$$
\begin{aligned}
D_{n}(f)= & \exp \left\{n V_{0}+\sum_{k=1}^{\infty} k V_{k} V_{-k}\right\} \prod_{j=0}^{m} b_{+}\left(z_{j}\right)^{-\left(\alpha_{j}-\beta_{j}\right)} b_{-}\left(z_{j}\right)^{-\left(\alpha_{j}+\beta_{j}\right)} \\
& \times n^{\sum_{j=0}^{m}\left(\alpha_{j}^{2}-\beta_{j}^{2}\right)} \prod_{0 \leq j<k \leq m}\left|z_{j}-z_{k}\right|^{2\left(\beta_{j} \beta_{k}-\alpha_{j} \alpha_{k}\right)}\left(\frac{z_{k}}{z_{j} e^{i \pi}}\right)^{\alpha_{j} \beta_{k}-\alpha_{k} \beta_{j}} \prod_{j=0}^{m} G_{\alpha_{j}, \beta_{j}}(1+o(1)) .
\end{aligned}
$$

Here, the product over $j<k$ is set to 1 if $m=0$ and

$$
G_{\alpha_{j}, \beta_{j}}=\frac{G\left(1+\alpha_{j}+\beta_{j}\right) G\left(1+\alpha_{j}-\beta_{j}\right)}{G\left(1+2 \alpha_{j}\right)}
$$

where $G$ is the Barnes' $G$-function.

In [6, 8, Theorem 1 is proved using a Riemann-Hilbert approach and generalized for less smooth functions $V$ of finite degree of smoothness satisfying the condition

$$
\sum_{k=-\infty}^{\infty}|k|^{s}\left|V_{k}\right|<\infty
$$

for $s$ such that

$$
s>\frac{1+\sum_{j=0}^{m}\left[\left(\Im \alpha_{j}\right)^{2}+\left(\operatorname{Re} \beta_{j}\right)^{2}\right]}{1-\|\beta\|} .
$$

In [6], the authors also prove the result for $\|\beta\|=1$ if $V(z) \in C^{\infty}(\mathbb{T})$, i.e. the generalised F-H, or Tracy-Basor conjecture which stems from F-H representations.

1.2. Basor-Tracy conjecture and Fisher-Hartwig representations. Basor and Tracy [1] computed the asymptotics of Toeplitz determinants with a symbol $f$ of two jump singularities at $z_{0}=1$ and $z_{1}=e^{i \pi}=-1$ with $\beta_{0}=1 / 2$ and $\beta_{1}=-1 / 2$, so that the seminorm, $\|\beta\|=1$. They noticed that the asymptotic behavior was not of the form in (10), but a linear combination of asymptotics (10) for two different Fisher-Hartwig determinants. The symbol in the first set of asymptotics corresponded to the original $f$ and the other to the symbol with jumps at 1 and -1 with $\beta_{0}=-1 / 2$ and $\beta_{1}=1 / 2$ respectively. The symbol of the second set of asymptotics only differed from the original one by a constant. This is a property of Fisher-Hartwig symbols called Fisher-Hartwig representations (see the definition and discussion below). This property applies to any $\mathrm{F}-\mathrm{H}$ symbol, however it only manifests itself in the asymptotics when $\|\beta\|=1$.

Definition 2. Let $f$ be Fisher-Hartwig. If $\alpha_{j} \neq 0$ or $\beta_{j} \neq 0$ or both are nonzero, we replace $\beta_{j}$ by $\beta_{j}+n_{j}=: \hat{\beta}_{j}, n_{j} \in \mathbb{Z}$, where $n_{j}$ satisfy the condition $\sum_{j=0}^{m} n_{j}=0$, but are otherwise arbitrary integers. The resulting function $f\left(z ; n_{0}, \ldots, n_{m}\right)$ is called a Fisher-Hartwig representation of $f$.

All Fisher-Hartwig representations of the symbol $f$ differ only by multiplicative constants, and it follows that

$$
f(z)=\prod_{j=0}^{m} z_{j}^{n_{j}} \times f\left(z ; n_{0}, \ldots, n_{m}\right) .
$$

We denote by $\mathcal{M}$ the set of all Fisher-Hartwig representations of $f$ for which

$$
\sum_{j=0}^{m}\left(\operatorname{Re} \beta_{j}+n_{j}\right)^{2}
$$


is minimal. Note that $\mathcal{M}$ is finite and there is an algorithm for describing $\mathcal{M}$ explicitly (see the proof of Lemma 1.12 of [6]). A Fisher-Hartwig representation is called degenerate if $\alpha_{j}+\left(\beta_{j}+n_{j}\right)$ or $\alpha_{j}-\left(\beta_{j}+n_{j}\right)$ is a negative integer for some $j$. The set $\mathcal{M}$ is called nondegenerate if it contains no degenerate Fisher-Hartwig representations.

Lemma 3. For $\beta=\left(\beta_{0}, \beta_{1}, \ldots, \beta_{m}\right)$, define the orbit of $\beta$ by

$$
O_{\beta}=\left\{\hat{\beta}: \hat{\beta}_{j}=\beta_{j}+n_{j}, \sum_{j=0}^{m} n_{j}=0\right\} .
$$

Then one and only one of the following conditions holds:

(i) There is a $\hat{\beta} \in O_{\beta}$ such that $\|\beta\|<1$, in which case $\hat{\beta}$ is unique and it is the unique element of $\mathcal{M}=\{\hat{\beta}\}$.

(ii) There is a $\hat{\beta} \in O_{\beta}$ such that $\|\beta\|=1$, in which case there are at least two such $\hat{\beta}$ parameters and all of them are obtained from each other by a repeated application of the following rule: add 1 to a $\hat{\beta}_{j}$ with the smallest real part and subtract 1 from a $\hat{\beta}_{j}$ with the largest. Moreover, $\mathcal{M}=\left\{\hat{\beta} \in O_{\beta}:\|\beta\|=1\right\}$.

All of the representations of the symbol $f$ with $\|\beta\|=1$, which correspond to all permutations of the $\beta$-parameters on the boundary of the strip $-1 / 2+q<\operatorname{Re} z<1 / 2+q$ for some $q \in \mathbb{R}, z \in \mathbb{C}$, contribute to the final asymptotics. The Basor-Tracy conjecture was finally proved in [6].

Theorem 4. (Basor-Tracy Conjecture) Let $f$ be Fisher-Hartwig, $\operatorname{Re} \alpha_{j}>-1 / 2, \beta_{j} \in \mathbb{C}, j=$ $0, \ldots, m$. Let $\mathcal{M}$ be nondegenerate. Then, as $n \rightarrow \infty$,

$$
D_{n}(f)=\sum_{\mathcal{M}}\left(\prod_{j=0}^{m} z_{j}^{n_{j}}\right)^{n} \mathcal{R}\left(f\left(z ; n_{0}, \ldots, n_{m}\right)\right)(1+o(1)),
$$

where each $\mathcal{R}\left(f\left(z ; n_{0}, \ldots, n_{m}\right)\right)$ is given by Fisher-Hartwig asymptotics for $D_{n}\left(f\left(z ; n_{0}, \ldots, n_{m}\right)\right)$ in (10) (without the error term).

1.3. Double-scaling limits. Let $f_{t} \in L^{1}$ for $t \geq 0$. By a double-scaling limit of Toeplitz determinants $D_{n}\left(f_{t}\right)$ we mean an asymptotic expansion of $D_{n}\left(f_{t}\right)$ valid uniformly for $0 \leq t<t_{0}$ for some sufficiently small $t_{0}$, as $n \rightarrow \infty$. In general, double-scaling limits (of not necessarily Toeplitz determinants) are of great interest in mathematics and in physics. We mention two previous results that are closely related to our present work. In [3], the double-scaling limits of Toeplitz determinants $D_{n}\left(f_{t}\right)$ were computed in the case that $f_{t}$ is sufficiently smooth for $t>0$ (as in the SSLT) and $f_{0}$ has one Fisher-Hartwig singularity. In [4], the double-scaling limits of $D_{n}\left(f_{t}\right)$ were computed in the case that $f_{t}$ has two Fisher-Hartwig singularities at $e^{i t}$ and $e^{-i t}$ when $t>0$ and $f_{0}$ has one FisherHartwig singularity at 1 . Both of these results have important applications, e.g., in random matrix theory and mathematical physics. See also [28] for discussion on double-scaling limits. Our work is concerned with the double-scaling limits of $D_{n}\left(f_{t}\right)$ in the case that $f_{t}$ has one fixed Fisher-Hartwig singularity on $\mathbb{T} \backslash\{1\}$ independent of $t$ and one additional Fisher-Hartwig singularity emerges as $t \rightarrow 0$. For the precise statements of our main results, see Section 4. In the next section we discuss applications to quantum spin chain models, and in particular we describe the phase transitions of the XY model using double-scaling limits of Toeplitz determinants.

\section{Applications}

2.1. The Ising model. In 1924 Ernst Ising showed that the one-dimensional Ising model with nearest-neighbor forces exhibits no phase transition; see [23, Chapter III]. The difference between 
the one-dimensional and two-dimensional Ising models is enormous. In one dimension both the free energy and the 2-spin correlation function can be exactly computed in closed form in the presence of a magnetic field $h$. By contrast, in two dimensions we need to rely on approximations. In the 1950s, Onsager and Kaufman studied the 2-spin correlation function $\left\langle\sigma_{00} \sigma_{n n}\right\rangle$ of the twodimensional Ising model (with the cyclic boundary conditions on the $2 M \times 2 N$ lattice and $h=0$ ) whose magnetization $M$ is given by

$$
M^{2}=\lim _{n \rightarrow \infty}\left\langle\sigma_{00} \sigma_{n n}\right\rangle=\lim _{n \rightarrow \infty} \lim _{M, N \rightarrow \infty} \frac{1}{Z(T)} \sum_{\{\sigma\}} \sigma_{00} \sigma_{n n} e^{-E(\{\sigma\}) / T},
$$

where

$$
E(\{\sigma\})=\sum_{j=-M}^{M-1} \sum_{k=-N}^{N-1}\left(J_{1} \sigma_{j k} \sigma_{j k+1}+J_{2} \sigma_{j k} \sigma_{j+1 k}\right)
$$

is the total energy corresponding to each spin configuration $\sigma$ given by $\sigma_{j k} \in\{ \pm 1\}, J_{1}, J_{2}>0$, and

$$
Z(T)=\sum_{\{\sigma\}} e^{-E(\{\sigma\}) / T}
$$

is the partition function at temperature $T$. For further details on the 2D Ising model, see [23].

As seen above, the correlation function is a huge sum of products and it is a remarkable fact that it can be expressed as a Toeplitz determinant. More precisely, using the notation in [3],

$$
\left\langle\sigma_{00} \sigma_{n n}\right\rangle=e^{n t / 2} D_{n}\left(f_{t}\right)
$$

where

$$
f_{t}(z)=\left(z-e^{t}\right)^{-1 / 2}\left(z-e^{-t}\right)^{1 / 2} z^{-1 / 2} e^{i \pi / 2}, \quad e^{t}=\sinh \frac{2 J_{1}}{T} \sinh \frac{2 J_{2}}{T} .
$$

We denote the critical temperature of the system by $T_{c}$. For the symbol $f$ in $(20)$, temperatures $T<T_{c}$ correspond to $t>0 ; T=T_{c}$ to $t=0$; and $T>T_{c}$ to $t<0$. Suppose first that $t>0$. Then

$$
\left(\log f_{t}\right)_{ \pm k}= \pm \frac{1}{2 k} e^{-t k} \quad \text { and } \quad\left(\log f_{t}\right)_{0}=-\frac{1}{2} t .
$$

Using the strong Szegő limit theorem, see (4), we can easily compute the magnetization for temperatures $T<T_{c}$ and obtain

$$
M^{2}=\left(1-e^{-2 t}\right)^{1 / 4}(1+o(1))=\left(1-\left(\sinh \frac{2 J_{1}}{T} \sinh \frac{2 J_{2}}{T}\right)^{-2}\right)^{1 / 4}(1+o(1))
$$

as $n \rightarrow \infty$.

When $t=0, f_{t}\left(e^{i \theta}\right)=e^{-i \frac{1}{2}(\theta-\pi)}$ has a Fisher-Hartwig singularity, and Theorem 1 implies

$$
D_{n}(0)=C n^{-1 / 4}(1+o(1))
$$

as $n \rightarrow \infty$, where $C=\sqrt{\pi} G\left(\frac{1}{2}\right)^{2}$ and $G$ is the Barnes' $G$-function.

To describe the phase transition of the 2D Ising model, one needs to compute the transition asymptotics of $D_{n}\left(f_{t}\right)$ as both $n \rightarrow \infty$ and $t \rightarrow 0$ simultaneously. These asymptotics were computed in [3] and also follow from our main result by setting $\alpha_{1}=\beta_{1}=0$. 


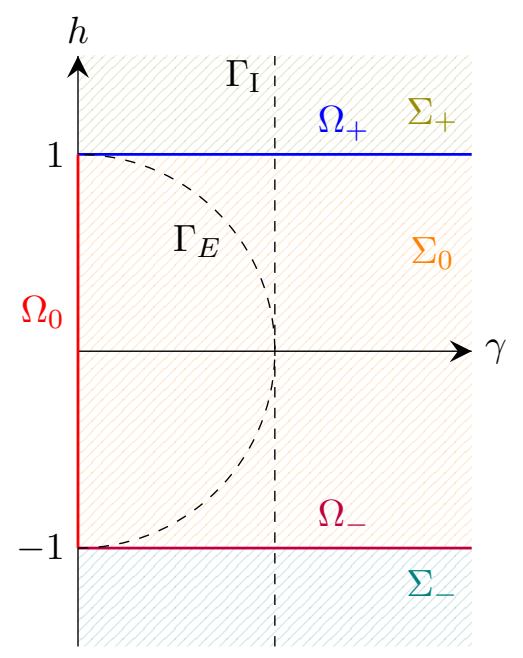

Figure 1. Phase diagram of the XY model for $\gamma \geq 0$. There are three critical lines: $\Omega_{+}: h=1, \Omega_{0}: \gamma=0,|h|<1, \Omega_{-}: h=-1$ and three non-critical domains: $\Sigma_{+}$: $h<-1, \Sigma_{0}:-1<h<1, \Sigma_{-}: h>1$. The line $\Gamma_{\text {I }}$ when $\gamma=1$ represents the Ising model in transverse magnetic field. On the line $\Gamma_{E}$ when $\gamma^{2}+h^{2}=1$ the ground state of the theory is a product of single spin states.

2.2. The XY model. The one-dimensional XY model [14], which is a generalization of the 1D Ising model, was introduced by Matsubara and Matsuda [22] in 1956 as a model of a quantum lattice gas. It is one of the simplest nontrivial integrable models with rich phase-diagram where most quantities can be calculated exactly. The critical behavior of this model was investigated in detail between 1968 and 1974 by Betts and his collaborators, who emphasized the relevance of this model to the study of insulating ferromagnets. See also [21]. The XY model has gathered a lot of attention within groups studying quantum entanglement with works such as Vidal et al. 27], Jin and Korepin [19], and Keating and Mezzadri [20]. For further references on the XY model in statistical mechanics, see the references in the recent monograph of Franchini [14].

In [15, Franchini and Abanov investigated how the change between regions over critical lines in the phase diagram (see Figure 1) influences the asymptotics of a special correlator called the Emptiness Formation Probability (EFP) for the 1-dimensional, anisotropic XY spin-1/2 chain in a transverse magnetic field $h$. The Hamiltonian of this model is given by

$$
H=\sum_{i=1}^{N}\left[\left(\frac{1+\gamma}{2}\right) \sigma_{i}^{x} \sigma_{i+1}^{x}+\left(\frac{1-\gamma}{2}\right) \sigma_{i}^{y} \sigma_{i+1}^{y}\right]-h \sum_{i=1}^{N} \sigma_{i}^{z},
$$

where $\sigma_{i}^{\alpha}$, with $\alpha=x, y, z$ are the Pauli matrices, which describe the spin operators on the $i$ th lattice site of the spin chain and the boundary conditions are chosen to be periodic; that is, $\sigma_{i}^{\alpha}=\sigma_{i+N}^{\alpha}$ with $(N>>1)$. The EFP is given by,

$$
P(n) \equiv \frac{1}{Z} \operatorname{Tr}\left\{e^{H / T} \prod_{j=1}^{n} \frac{1-\sigma_{i}^{z}}{2}\right\}
$$


where $Z=\operatorname{Tr}\left\{e^{H / T}\right\}$ is the partition function and $T$ is the temperature. The majority of the paper [15] deals with the case when $T=0$. In that case,

$$
P(n) \equiv\left\langle 0\left|\prod_{i=1}^{n} \frac{1-\sigma_{i}^{z}}{2}\right| 0\right\rangle,
$$

and $P(n)$ is then the probability that $n$ consecutive spins are all aligned downward in the ground state $|0\rangle$.

After reformulating (21), using spinless fermions and other transformation techniques of statistical mechanics, the authors arrive at fermionic correlators in the thermodynamic limit given by

$$
F_{j k} \equiv i\left\langle\psi_{j} \psi_{k}\right\rangle=-i\left\langle\psi_{j}^{*} \psi_{k}^{*}\right\rangle=\int_{0}^{2 \pi} \frac{1}{2} \sin \vartheta_{q} e^{i q(j-k)} \frac{d q}{2 \pi}
$$

and

$$
G_{j k} \equiv\left\langle\psi_{j} \psi_{k}^{*}\right\rangle=\int_{0}^{2 \pi} \frac{1+\cos \vartheta_{q}}{2} e^{i q(j-k)} \frac{d q}{2 \pi},
$$

where $\psi_{i}$ are the spinless fermions (cf. Jordan-Wigner transformation),

$$
e^{i \vartheta_{q}}=\frac{1}{\varepsilon_{q}}(\cos q-h+i \gamma \sin q)
$$

and

$$
\varepsilon_{q}=\sqrt{(\cos q-h)^{2}+\gamma^{2} \sin ^{2} q}
$$

The EFP can be expressed as

$$
P(n)=\operatorname{Pf}(\mathbf{M})
$$

where

$$
\operatorname{Pf}(M) \equiv \sum_{P}(-1)^{P} M_{p_{1} p_{2}} M_{p_{3} p_{4}} \ldots M_{p_{2 n-1} p_{2 n}}
$$

is called the Pfaffian. Here the sum is taken over all possible permutations $P=\left\{p_{1}, p_{2}, \ldots, p_{2 n}\right\}$ of the set $\{1,2, \ldots, n\}$ and $(-1)^{P}$ denotes the parity of the permutation. The matrix $\mathbf{M}$ is a $2 n \times 2 n$ skew-symmetric matrix of correlation functions given by

$$
\mathbf{M}=\left(\begin{array}{cc}
-i \mathbf{F} & \mathbf{G} \\
-\mathbf{G} & i \mathbf{F}
\end{array}\right)
$$

where $\mathbf{F}$ and $\mathbf{G}$ are $n \times n$ matrices with entries given by $F_{j k}$ and $G_{j k}$ in $(23)$ and $(24)$. One of the properties of the Pfaffian gives that

$$
P(n)=\operatorname{Pf}(\mathbf{M})=\sqrt{\operatorname{det}(\mathbf{M})}
$$

After performing a unitary transformation one arrives at

$$
\mathbf{M}^{\prime}=\mathbf{U M U}^{*}=\left(\begin{array}{cc}
\mathbf{0} & \mathbf{S}_{n} \\
-\mathbf{S}_{n}^{*} & \mathbf{0}
\end{array}\right), \quad \mathbf{U}=\frac{1}{\sqrt{2}}\left(\begin{array}{cc}
\mathbf{I} & -\mathbf{I} \\
\mathbf{I} & \mathbf{I}
\end{array}\right),
$$

where $\mathbf{I}$ is a unit $n \times n$ matrix and $\mathbf{S}_{n}=\mathbf{G}+i \mathbf{F}, \mathbf{S}_{n}^{*}=\mathbf{G}-i \mathbf{F}$. The unitary transformation does not change the determinant and so

$$
\operatorname{det}(\mathbf{M})=\operatorname{det}\left(\mathbf{M}^{\prime}\right)=\operatorname{det}\left(\mathbf{S}_{n}\right) \operatorname{det}\left(\mathbf{S}_{n}^{*}\right)=\left|\operatorname{det}\left(\mathbf{S}_{n}\right)\right|^{2} .
$$

Thus,

$$
P(n)=\left|\operatorname{det}\left(\mathbf{S}_{n}\right)\right|,
$$


where the matrix $\mathbf{S}_{n}$ is an $n \times n$ Toeplitz matrix with the symbol

$$
\sigma(q)=\frac{1}{2}+\frac{\cos q-h+i \gamma \sin q}{2 \sqrt{(\cos q-h)^{2}+\gamma^{2} \sin ^{2} q}},
$$

$q \in(0,2 \pi]$ and $h$ is the external magnetic field (for critical lines in the phase diagram, see Figure 1 ). If $\gamma=0$, the problem is isotropic and exhibits a Gaussian behavior, instead of exponential, which can be seen using Widom's theorem with a power law prefactor (see [24]). Suppose that $\gamma \neq 0$. Since $\mathbf{S}_{n}=\mathbf{G}+i \mathbf{F},(23)$ and (24) imply that

$$
\sigma(q)=\frac{1}{2}\left(1+e^{i \vartheta_{q}}\right), \quad e^{i \vartheta_{q}}=\cos \vartheta_{q}+i \sin \vartheta_{q},
$$

from which we obtain (27) according to $(25)$ and $(26)$.

2.2.1. Region $\Sigma_{-}$corresponding to $h<-1$. In this region, for $\gamma \neq 0$ and $h<-1$, the symbol (27) is analytic for all $q$ and we can use the SSLT (4) to get the asymptotics. We write $q(\theta)$ for $q\left(e^{i \theta}\right)$ and we may emphasize the dependence on $h$ or $t$ by writing $\sigma(\theta, h)$ or $\sigma(\theta, t)$ respectively. In this region, if $h=h(t)=-e^{t}$ with $t>0$, then, as $t \rightarrow 0, h$ approaches -1 from $h<-1$; that is, transition from $\Sigma_{-}$to $\Sigma_{0}$.

We can factorise another analytic function (for $t>0$ ) out of $\sigma(\theta, t)$,

$$
\mathcal{T}_{-1}(\theta, t)=\left(e^{i \theta}+e^{t}\right) e^{-3 \pi i / 2}, \quad \theta \in(0,2 \pi),
$$

to write simply

$$
\sigma(\theta, t)=\mathcal{T}_{-1}(\theta, t) e^{V(\theta, t)},
$$

where $e^{V(\theta, t)}$ is an analytic function left after the factorization. Function $e^{V(\theta, t)}$ depends on $t$, but the limit $t \rightarrow 0$ does not affect its analyticity. Observe that $\mathcal{T}_{-1}(\theta, t) \rightarrow \mathcal{F}_{-1}(\theta)$ as $t \rightarrow 0$, where

$$
\mathcal{F}_{-1}(\theta)=\left|e^{i \theta}+1\right| e^{i \theta / 2} g_{-1,1 / 2}\left(e^{i \theta}\right) e^{-i \pi / 2},
$$

and $g_{z_{j}, \beta_{j}}(z)$ is defined in (6). The function $\mathcal{F}_{-1}(\theta, t)$ has a pure Fisher-Hartwig singularity at $z=-1$ with $\alpha_{1}=1 / 2$ and $\beta_{1}=1 / 2$. Using (84) and (88) with $\theta_{1}=\pi$, we get

$$
\begin{aligned}
\mathcal{T}_{-1}(\theta, 0) & =\left(e^{i \theta}+1\right)^{2(1 / 2)} e^{-i \theta / 2} e^{-\pi i} g_{-1,-1 / 2}\left(e^{i \theta}\right) g_{-1,1 / 2}\left(e^{i \theta}\right) e^{-\pi i / 2} e^{i \theta / 2} \\
& =\frac{\left(e^{i \theta}+1\right)^{2(1 / 2)}}{\left(e^{-i \theta} e^{i l_{1}}\right)^{1 / 2}} g_{-1,1 / 2}\left(e^{i \theta}\right) e^{-\pi i / 2} e^{i \theta / 2}=\mathcal{F}_{-1}(\theta)
\end{aligned}
$$

since $e^{i l_{1}(-1 / 2)}=e^{-\pi i} g_{-1,-1 / 2}\left(e^{i \theta}\right)$, where $l_{1}$ is as in (84). Thus, we cross the critical line $h=-1$ and arrive at the next non-critical region $\Sigma_{0}$.

2.2.2. Region $\Sigma_{0}$ corresponding to $-1<h<1$. For $-1<h<1$ the symbol has one Fisher-Hartwig singularity at $\theta=\pi$ with strength $\beta=\frac{1}{2}, \alpha=\frac{1}{2}$ as seen from (31). We can factorize the singularity out of $\sigma(\theta, h)$. The function $\mathcal{F}_{-1}^{-1}(\theta) \sigma(\theta, h)$ is analytic for $-1<h<1$ as shown on the plots in Figure 3 .

As $h \rightarrow 1$ we see an emergence of a second singularity at $z=1$ or $\theta=0$. In this region we can think of $h=e^{-t}$ with $t>0$ (note that $t$ here is different from the one in the previous region), and $t \rightarrow 0$ corresponds to the limit as $h$ approaches 1 from $h<1$. By taking the limit $t \rightarrow 0$ we arrive at the region $\Sigma_{+}$. As in $\Sigma_{-}$, we define

$$
\mathcal{T}_{1}(\theta, t)=\left(e^{i \theta}-e^{-t}\right) e^{-i \theta}, \quad \theta \in(0,2 \pi) .
$$




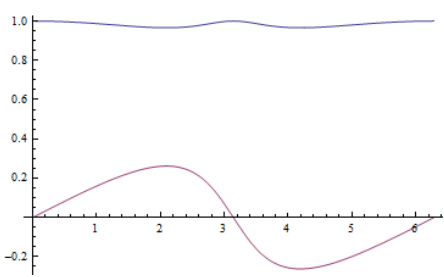

(A) $h=-2$

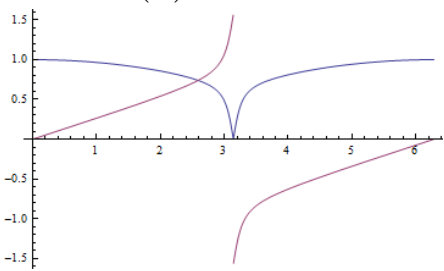

(D) $h=-0.9$

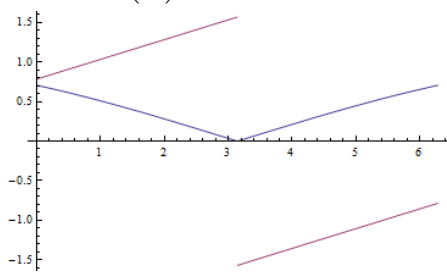

(G) $h=1$

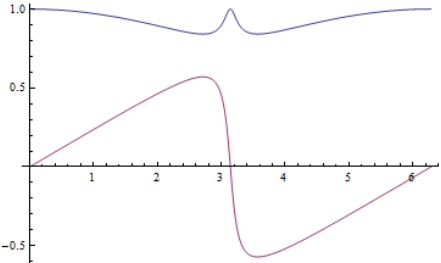

(в) $h=-1.1$

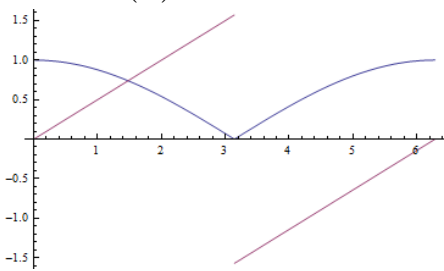

(E) $h=0$

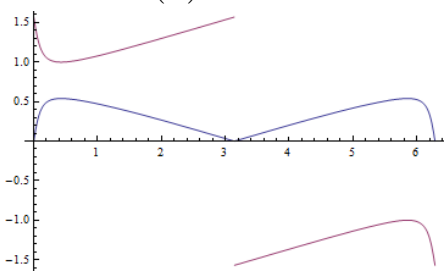

(н) $h=1.1$

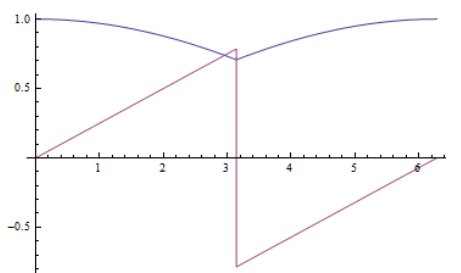

(c) $h=-1$

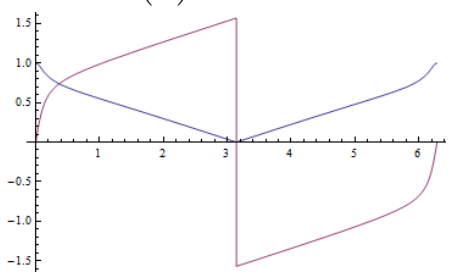

(F) $h=0.9$

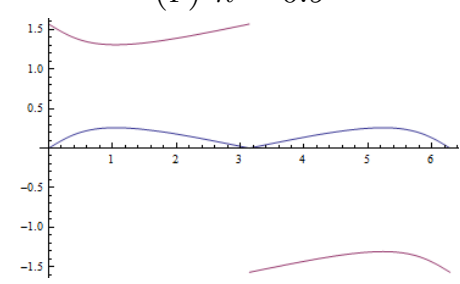

(I) $h=2$

Figure 2. Plots of $|\sigma(\theta, h)|$ (blue) and $\arg \sigma(\theta, h)$ (red) for different values of $h$ with $\gamma=1$

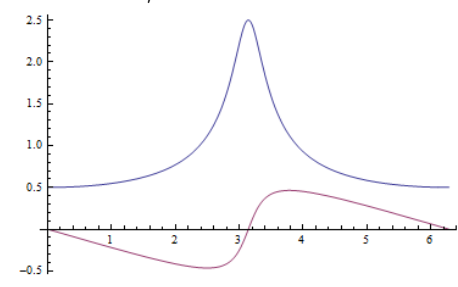

(A) $h=-0.8$

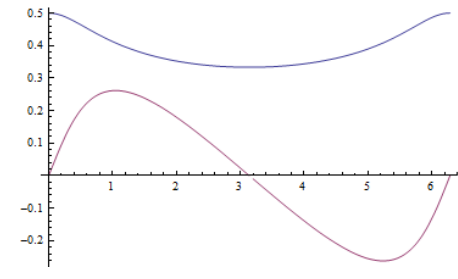

(в) $h=0.5$

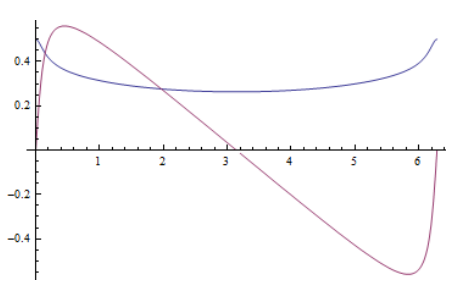

(c) $h=0.9$

Figure 3. Plots of $\left|\mathcal{F}_{-1}^{-1}(\theta) \sigma(\theta, h)\right|$ (blue) and $\arg \mathcal{F}_{-1}^{-1}(\theta) \sigma(\theta, h)$ (red) with $\gamma=1$; showing no jumps in argument and boundedness, and non-vanishing in absolute value for $-1<h<1$.

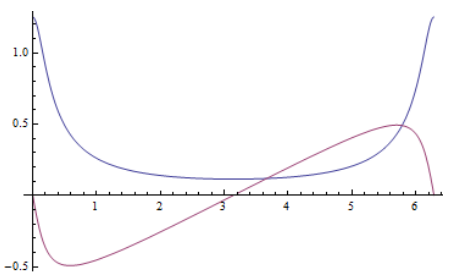

(A) $h=1.2$

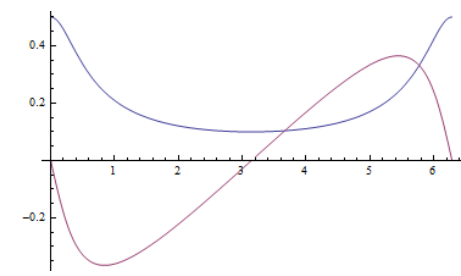

(в) $h=1.5$

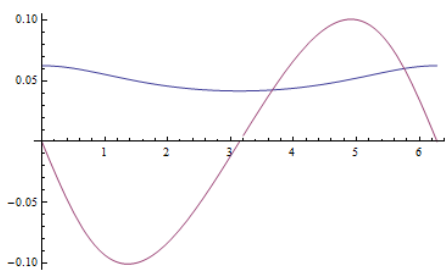

(c) $h=5$

Figure 4. Plots of $\left|\mathcal{F}_{-1}^{-1}(\theta) \mathcal{F}_{1}^{-1}(\theta) \sigma(\theta, h)\right|$ (blue) and $\arg \mathcal{F}_{-1}^{-1}(\theta) \mathcal{F}_{1}^{-1}(\theta) \sigma(\theta, h)$ (red) with $\gamma=1$; showing no jumps in argument and boundedness, and nonvanishing in absolute value for $h>1$. 
Compare this function to $a_{t}$ in $(38)$ with $\alpha_{0}=1 / 2$ and $\beta_{0}=-1 / 2$. We can write

$$
\sigma(\theta, t)=\mathcal{T}_{1}(\theta, t) \mathcal{F}_{-1}(\theta) e^{\widetilde{V}(\theta, t)}
$$

where $e^{\widetilde{V}(\theta, t)}$ is an analytic function left after the factorization. Notice that this is exactly the symbol in (38) below with $z_{1}=-1, \alpha_{0}=1 / 2, \beta_{0}=-1 / 2, \alpha_{1}=1 / 2, \beta_{1}=1 / 2$. Again, $e^{\widetilde{V}(\theta, t)}$ depends on $t$, but the limit $t \rightarrow 0$ does not affect its analyticity. As $t \rightarrow 0$,

$$
\mathcal{T}_{1}(\theta, t) \rightarrow \mathcal{F}_{1}(\theta)=\left|e^{i \theta}-1\right| e^{-i \theta / 2} e^{i \pi / 2} .
$$

Observe that $\mathcal{F}_{1}(\theta, t)$ with (5) has a pure singularity at $z=1$ with $\alpha_{0}=1 / 2$ and $\beta_{0}=-1 / 2$. Using (84) again, we get

$$
\mathcal{T}_{1}(\theta, 0)=\frac{\left(e^{i \theta}+1\right)^{2(1 / 2)}}{\left(e^{i \theta} e^{i \pi}\right)^{1 / 2}} e^{-i \theta / 2} e^{i \pi / 2}=\mathcal{F}_{1}(\theta)
$$

Thus, we cross the critical line $h=1$ and arrive at the next non-critical region $\Sigma_{+}$.

2.2.3. Region $\Sigma_{+}$corresponding to $h>1$. For $h>1$, the symbol $\sigma(\theta, h)$ has two Fisher-Hartwig singularities at $\theta=0$ and $\theta=\pi$. Let $\theta_{0}=0$ and $\theta_{1}=\pi$. The corresponding strengths are $\beta_{0}=-\frac{1}{2}$, $\alpha_{0}=\frac{1}{2}, \beta_{1}=\frac{1}{2}, \alpha_{1}=\frac{1}{2}$. Using the notation in (31) and (34), we can write the symbol as

$$
\sigma(\theta, t)=\mathcal{F}_{-1}(\theta, t) \mathcal{F}_{1}(\theta, t) e^{\widehat{V}(\theta, h)} .
$$

To see $e^{\widehat{V}(\theta, h)}$ is analytic for $h>1$, we look at the plot of $\mathcal{F}_{-1}^{-1}(\theta) \mathcal{F}_{1}^{-1}(\theta) \sigma(\theta, h)$ in Figure 4 and notice that the function has no jumps in its argument and its absolute value does not vanish or blow up.

However, this symbol has another representation, which corresponds to the seminorm of $\beta$ parameters; that is, if

$$
\mathcal{F}_{-1}^{\hat{\beta}}(\theta)=\left|e^{i \theta}+1\right| e^{-i \theta / 2} g_{-1,-1 / 2}\left(e^{i \theta}\right) e^{i \pi / 2},
$$

and

$$
\mathcal{F}_{1}^{\hat{\beta}}(\theta)=\left|e^{i \theta}-1\right| e^{i \theta / 2} e^{-i \pi / 2},
$$

then

$$
\sigma(\theta, t)=e^{-i \pi} \mathcal{F}_{-1}^{\hat{\beta}}(\theta, t) \mathcal{F}_{1}^{\hat{\beta}}(\theta, t) e^{\widehat{V}(\theta, h)} .
$$

By the Tracy-Basor conjecture, the asymptotics of the Toeplitz determinant are obtained from the two representations according to Theorem 4.

2.2.4. Transition between the regions. It is natural to seek information about the transition between $\Sigma_{0}$ and each of the two regions $\Sigma_{ \pm}$, and describe what happens close to the critical points. As mentioned above, the results of [3] provide uniform asymptotics of the Toeplitz determinant with a symbol that can be written in the form (30) after rotating the problem in [3] by $\pi$. This transition can thus be used to describe the change between the regions $\Sigma_{-}$and $\Sigma_{0}$. Our results describe the transition between $\Sigma_{0}$ and $\Sigma_{+}$, which involve the emergence of an additional singularity and $\|\beta\|=1$ on the critical line. This transition is described in Theorem 6 with $z_{1}=-1, \alpha_{0}=1 / 2$, $\beta_{0}=-1 / 2, \alpha_{1}=1 / 2, \beta_{1}=1 / 2$, which is exactly the symbol in $(33)$ above. 


\section{The Symbol}

We start with one Fisher-Hartwig singularity positioned at the point $z_{1}=e^{i \theta_{1}}, \theta_{1} \in(0,2 \pi)$ and one that emerges, at $z_{0}=1$ as $t \rightarrow 0$. Locally, around the point $z=1$, we have the situation which was described in $[3]$. For $t \geq 0$ and $z \in \mathbb{T}$, we write the symbol as follows:

$$
\begin{aligned}
f_{t}(z) & =f(z ; t)=a_{t}(z) \times z^{\beta_{1}}\left|z-z_{1}\right|^{2 \alpha_{1}} g_{z_{1}, \beta_{1}}(z) z_{1}^{-\beta_{1}} \\
& =e^{V(z)}\left(z-e^{t}\right)^{\alpha_{0}+\beta_{0}}\left(z-e^{-t}\right)^{\alpha_{0}-\beta_{0}} z^{-\alpha_{0}+\beta_{0}} e^{-i \pi\left(\alpha_{0}+\beta_{0}\right)} \times z^{\beta_{1}}\left|z-z_{1}\right|^{2 \alpha_{1}} g_{z_{1}, \beta_{1}}(z) z_{1}^{-\beta_{1}}
\end{aligned}
$$

where

$$
a_{t}(z)=e^{V(z)}\left(z-e^{t}\right)^{\alpha_{0}+\beta_{0}}\left(z-e^{-t}\right)^{\alpha_{0}-\beta_{0}} z^{-\alpha_{0}+\beta_{0}} e^{-i \pi\left(\alpha_{0}+\beta_{0}\right)}
$$

has an emerging singularity at $t=0$ with strengths $\alpha_{0}$ and $\beta_{0}$, and

$$
e^{V(z)}\left(z-e^{t}\right)^{\alpha_{0}+\beta_{0}}\left(z-e^{-t}\right)^{\alpha_{0}-\beta_{0}} z^{-\alpha_{0}+\beta_{0}} e^{-i \pi\left(\alpha_{0}+\beta_{0}\right)}
$$

is analytic in $\mathbb{C} \backslash\left(\left[0, e^{-t}\right] \cup\left[e^{t},+\infty\right]\right)$ for $t>0$. Also, note that for $t>0, f_{t}$ has one Fisher-Hartwig singularity with strengths $\alpha_{1}$ and $\beta_{1}$; while for $t=0, f_{t}$ has two singularities with strengths $\alpha_{j}$ and $\beta_{j}$ for $j=0,1$. In both cases, $f_{t}$ can be represented in the form (5).

Using Fisher-Hartwig asymptotics, it is easy to find the asymptotics of $D_{n}\left(f_{t}\right)$ for a fixed $t$. Indeed, the Fourier coefficients of $\log a(z ; t)$ are given by

$$
\left(\log a_{t}\right)_{0}=V_{0}+t\left(\alpha_{0}+\beta_{0}\right) \quad \text { and } \quad\left(\log a_{t}\right)_{ \pm k}=\left(V_{ \pm k}-\left(\alpha_{0} \pm \beta_{0}\right) \frac{e^{-t k}}{k}\right)
$$

for $k \in \mathbb{N}$ and the Wiener-Hopf factorization of $a_{t}$ is given by

$$
\log a_{t}(z)=\log a_{t,+}(z)+\left(\log a_{t}(z)\right)_{0}+\log a_{t,-}(z),
$$

where

$$
\log a_{t,+}(z)=\sum_{k=1}^{\infty}\left(\log a_{t}(z)\right)_{k} z^{k}, \quad \log a_{t,-}(z)=\sum_{k=1}^{\infty}\left(\log a_{t}(z)\right)_{-k} z^{-k} .
$$

By Theorem 1, we have

$$
\begin{aligned}
D_{n}\left(f_{t}\right)= & \exp \left\{n\left(\log a_{t}(z)\right)_{0}\right\} \exp \left\{\sum_{k=1}^{\infty} k\left(\log a_{t}\right)_{k}\left(\log a_{t}\right)_{-k}\right\} \\
& \times \exp \left\{-\left(\alpha_{1}-\beta_{1}\right) \log a_{t,+}\left(z_{1}\right)-\left(\alpha_{1}+\beta_{1}\right) \log a_{t,-}\left(z_{1}\right)\right\} n^{\left(\alpha_{1}^{2}-\beta_{1}^{2}\right)} G_{\alpha_{1}, \beta_{1}}(1+o(1))
\end{aligned}
$$

as $n \rightarrow \infty$.

For the ease of notation in what follows, we denote the following interaction constants between two singularities (compare with (10),

$$
I\left(z_{j, k}, \beta_{j, k}\right):=\left|z_{j}-z_{k}\right|^{2\left(\beta_{j} \beta_{k}-\alpha_{j} \alpha_{k}\right)}\left(\frac{z_{k}}{z_{j} e^{i \pi}}\right)^{\alpha_{j} \beta_{k}-\alpha_{k} \beta_{j}} G_{\alpha_{j}, \beta_{j}} G_{\alpha_{k}, \beta_{k}}
$$

with $j<k$, and

$$
E\left(z_{j}, \beta_{j}\right):=\exp \left\{-\left(\alpha_{j}-\beta_{j}\right) V_{+}\left(z_{j}\right)-\left(\alpha_{j}+\beta_{j}\right) V_{-}\left(z_{j}\right)\right\} .
$$

When $t=0$, the symbol has two Fisher-Hartwig singularities and we have to consider two cases. If \|\|$\beta \|<1$, Theorem 1 implies that

$$
D_{n}(f)=\exp \left\{n V_{0}+\sum_{k=1}^{\infty} k V_{k} V_{-k}\right\} E\left(z_{0}, \beta_{0}\right) E\left(z_{1}, \beta_{1}\right) \times n^{\sum_{j=0}^{1}\left(\alpha_{j}^{2}-\beta_{j}^{2}\right)} I\left(z_{0,1}, \beta_{0,1}\right)(1+o(1)) .
$$

If $\|\beta\|=1$, the symbol possesses two Fisher-Hartwig representations (see Definition 2) and we need to apply Theorem 4 to obtain the asymptotics. The trivial representation corresponds to $\beta_{0}$ 
and $\beta_{1}$. Without loss of generality, we may assume $\operatorname{Re} \beta_{0}<\operatorname{Re} \beta_{1}$, in which case the nontrivial representation corresponds to $\tilde{\beta}_{0}=\beta_{0}+1$ and $\tilde{\beta}_{1}=\beta_{1}-1$. By (17),

$$
\begin{aligned}
& D_{n}(f)=\left[\exp \left\{n V_{0}+\sum_{k=1}^{\infty} k V_{k} V_{-k}\right\} E\left(z_{0}, \beta_{0}\right) E\left(z_{1}, \beta_{1}\right) n^{\sum_{j=0}^{1}\left(\alpha_{j}^{2}-\beta_{j}^{2}\right)} I\left(z_{0,1}, \beta_{0,1}\right)\right. \\
& \left.+\left(z_{1}^{-1}\right)^{n} \exp \left\{n V_{0}+\sum_{k=1}^{\infty} k V_{k} V_{-k}\right\} E\left(z_{0}, \tilde{\beta}_{0}\right) E\left(z_{1}, \tilde{\beta}_{1}\right) \times n^{\sum_{j=0}^{1}\left(\alpha_{j}^{2}-\tilde{\beta}_{j}^{2}\right)} I\left(z_{0,1}, \tilde{\beta}_{0,1}\right)\right](1+o(1)) .
\end{aligned}
$$

This case will be considered in more detail in Section 9 .

Notice that taking the limit as $t \rightarrow 0$ in (39) does not agree with (42). In the next section, we provide the asymptotic expansion for $D_{n}\left(f_{t}\right)$ that holds uniformly for all sufficiently small $t$.

\section{Statement of Results}

Our main results (Theorems 5 and 6) describe the asymptotic behavior of Toeplitz determinants $D_{n}\left(f_{t}\right)$ as $n \rightarrow \infty$ and $t \rightarrow 0$ simultanously, where the symbol $f_{t}$ is of the form

$$
\begin{aligned}
f_{t}(z) & =f(z ; t)=a_{t}(z) \times z^{\beta_{1}}\left|z-z_{1}\right|^{2 \alpha_{1}} g_{z_{1}, \beta_{1}}(z) z_{1}^{-\beta_{1}} \\
& =e^{V(z)}\left(z-e^{t}\right)^{\alpha_{0}+\beta_{0}}\left(z-e^{-t}\right)^{\alpha_{0}-\beta_{0}} z^{-\alpha_{0}+\beta_{0}} e^{-i \pi\left(\alpha_{0}+\beta_{0}\right)} \times z^{\beta_{1}}\left|z-z_{1}\right|^{2 \alpha_{1}} g_{z_{1}, \beta_{1}}(z) z_{1}^{-\beta_{1}},
\end{aligned}
$$

and $V$ is analytic in a neighborhood of the unit circle. Our results can be used to study the emptiness formation probability for the one-dimensional anisotropic XY spin chain in a transverse magnetic field, and in particular we obtain a full description of the transition between different regions in the phase diagram across critical lines - see Sections 2.2 and 2.2 .4 above for further details.

Theorem 5. Let $f_{t}$ be defined as above and suppose that $\alpha_{0}, \alpha_{1} \in \mathbb{C}$ with $\operatorname{Re} \alpha_{j}>-\frac{1}{2}, \beta_{0}, \beta_{1} \in \mathbb{C}$ with $\|\beta\|<1$. The following asymptotic expansion holds as $n \rightarrow \infty$ with the error term o(1) uniform for $0 \leq t \leq t_{0}$, where $t_{0}$ is sufficiently small,

$$
\begin{aligned}
D_{n}\left(f_{t}\right)= & \exp \left\{n V_{0}+n t\left(\alpha_{0}+\beta_{0}\right)\right\} \exp \left\{\sum_{k=1}^{\infty} k\left[\left(\log a_{t}\right)_{k}\right]\left[\left(\log a_{t}\right)_{-k}\right]\right\} \\
& \times \exp \left\{-\left(\alpha_{1}-\beta_{1}\right) \log a_{t,+}\left(z_{1}\right)\right\} \exp \left\{\left(\alpha_{1}+\beta_{1}\right) \log a_{t,-}\left(z_{1}\right)\right\} \\
& \times n^{\left(\alpha_{1}^{2}-\beta_{1}^{2}\right)} G_{\alpha_{0}, \beta_{0}} G_{\alpha_{1}, \beta_{1}} \tilde{\Omega}(2 n t)(1+o(1))
\end{aligned}
$$

as $n \rightarrow \infty$, where $G_{\alpha_{j}, \beta_{j}}$ is the product of Barnes $G$-functions, defined in (11), and

$$
\tilde{\Omega}(2 n t)=\exp \{\Omega(2 n t)\}=\exp \left\{\int_{0}^{2 n t} \frac{\sigma(x)-\alpha_{0}^{2}+\beta_{0}^{2}}{x} d x+\left(\alpha_{0}^{2}-\beta_{0}^{2}\right) \log 2 n t\right\} .
$$

The function $\sigma$ is a particular solution to the second order ODE, the Jimbo-Miwa-Okamoto $\sigma$-form [17, 18] of the Painlevé $V$ equation, with parameters depending on $\alpha_{0}$ and $\beta_{0}$. It is real analytic on $(0,+\infty)$, and its asymptotic behavior for $x>0$ is given by

$$
\sigma(x)= \begin{cases}\alpha_{0}^{2}-\beta_{0}^{2}+\frac{\alpha_{0}^{2}-\beta_{0}^{2}}{2 \alpha_{0}}\left\{x-x^{1+2 \alpha_{0}} C\left(\alpha_{0}, \beta_{0}\right)\right\}(1+\mathcal{O}(x)), & x \rightarrow 0,2 \alpha_{0} \notin \mathbb{Z}, \\ \alpha_{0}^{2}-\beta_{0}^{2}+\mathcal{O}(x)+\mathcal{O}\left(x^{1+2 \alpha_{0}}\right)+\mathcal{O}\left(x^{1+2 \alpha_{0}} \log x\right), & x \rightarrow 0,2 \alpha_{0} \in \mathbb{Z} \\ x^{-1+2 \alpha_{0}} e^{-x} \frac{1}{\Gamma\left(\alpha_{0}-\beta_{0}\right) \Gamma\left(\alpha_{0}+\beta_{0}\right)}(1+\mathcal{O}(1 / x)), & x \rightarrow+\infty\end{cases}
$$

where

$$
C\left(\alpha_{0}, \beta_{0}\right)=\frac{\Gamma\left(1+\alpha_{0}+\beta_{0}\right) \Gamma\left(1+\alpha_{0}-\beta_{0}\right) \Gamma\left(1-2 \alpha_{0}\right)}{\Gamma\left(1-\alpha_{0}+\beta_{0}\right) \Gamma\left(1-\alpha_{0}-\beta_{0}\right) \Gamma\left(+2 \alpha_{0}\right)^{2}} \frac{1}{1+2 \alpha_{0}}
$$


and $\Gamma(z)$ is the Euler's $\Gamma$-function.

The proof of this theorem is given in Section 8 .

Theorem 6. Let $\alpha_{0}, \alpha_{1} \in \mathbb{C}$ with $\operatorname{Re} \alpha_{j}>-\frac{1}{2}, \beta_{0}, \beta_{1} \in \mathbb{C}$ be such that $\|\beta\|=1$, and $f_{t}$ be defined by (38). Denote by $\tilde{\beta}_{0}=\beta_{0}+n_{0}, \tilde{\beta}_{1}=\beta_{0}+n_{1}$ the only non-trivial Fisher-Hartwig representation of $f_{0}$. The following asymptotic expansion holds as $n \rightarrow \infty$ with the error term o(1) uniform for $0 \leq t \leq t_{0}$, where $t_{0}$ is sufficiently small,

$$
\begin{aligned}
D_{n}\left(f_{t}\right)=\mathcal{R} & \left(f_{t}\left(z ; \beta_{0}, \beta_{1}\right)\right)+\left(z_{1}^{n_{1}}\right)^{n} \mathcal{R}\left(f_{t}\left(z ; \tilde{\beta}_{0}, \tilde{\beta}_{1}\right)\right) \\
& \times \frac{n^{-\left(2 \beta_{0}+1\right)}}{\Gamma\left(1+\alpha_{0}+\beta_{0}\right)} \frac{K(2 n t)}{e^{n t}}\left(1-e^{-2 t}\right)^{-\left(2 \beta_{0}+1\right)} \Sigma(t),
\end{aligned}
$$

where $\mathcal{R}\left(f_{t}\left(z ; \tilde{\beta}_{0}, \tilde{\beta}_{1}\right)\right)$ is given by 43 and $f_{t}\left(z ; \tilde{\beta}_{0}, \tilde{\beta}_{1}\right)$ is the symbol $f_{t}$ with jump strengths $\tilde{\beta}_{0}, \tilde{\beta}_{1}$. Further, the incomplete Gamma function (see [11, Eq. 8.2.2])

$$
K(x)=e^{x / 2} \int_{x}^{\infty} y^{\alpha_{0}+\beta_{0}} e^{-y} d y
$$

has the following behavior:

$$
K(x) \sim \begin{cases}e^{-x / 2} x^{\alpha_{0}+\beta_{0}}, & \text { as } x \rightarrow \infty, \\ e^{x / 2} \Gamma\left(\alpha_{0}+\beta_{0}+1\right), & \text { as } x \rightarrow 0,\end{cases}
$$

and

$$
\begin{aligned}
\Sigma(t)= & {\left[\left(\frac{z_{1}-e^{t}}{z_{1}-e^{-t}}\right)^{\alpha_{1}+\tilde{\beta}_{1}} \exp \left\{2 \sum_{k=1}^{\infty} V_{k}(\sinh (t k))\right\}\left(\frac{2 t}{1-e^{-2 t}}\right)^{\alpha_{0}-\beta_{0}}\right.} \\
& \left.+\left(\frac{z_{1}-e^{t}}{z_{1}-e^{-t}}\right)^{\alpha_{1}-\tilde{\beta}_{1}} \exp \left\{-2 \sum_{k=1}^{\infty} V_{-k}(\sinh (t k))\right\}\left(\frac{2 t}{1-e^{-2 t}}\right)^{-\left(\alpha_{0}+\beta_{0}\right)}\right] .
\end{aligned}
$$

\section{A Riemann-Hilbert problem For orthogonal polynomials}

Let $f \in L^{1}(\mathbb{T})$. A system of polynomials

$$
\phi_{n}(z)=\chi_{n} z^{n}+\ldots \quad \text { and } \quad \hat{\phi}_{n}(z)=\chi_{n} z^{n}+\ldots \quad(z \in \mathbb{T}, n=0,1, \ldots)
$$

is said to be orthonormal with respect to $f$ if for each $n$,

$$
\int_{\mathbb{T}} \phi_{n}(z) z^{-j} f(z) \frac{d z}{2 \pi i z}=\chi_{n}^{-1} \delta_{j n}, \quad \int_{\mathbb{T}} \hat{\phi}_{n}\left(z^{-1}\right) z^{j} f(z) \frac{d z}{2 \pi i z}=\chi_{n}^{-1} \delta_{j n},
$$

for $j=0, \ldots, n$, or, equivalently,

$$
\int_{\mathbb{T}} \phi_{n}(z) \hat{\phi}_{j}\left(z^{-1}\right) f(z) \frac{d z}{2 \pi i z}=\delta_{j n}
$$

for $j=0, \ldots, n$.

Note that the function $f$ is complex-valued and the existence of $\varphi_{n}$ is not guaranteed. However, it is well known that if $D_{n}(f) \neq 0$ for all $n \geq N_{0}$ with some $N_{0}$, then the polynomials $\phi_{n}$ and $\hat{\phi}_{n}$ 
satisfying (50) for $n \geq N_{0}$ exist and are given by

$$
\phi_{n}(z)=\frac{1}{\sqrt{D_{n} D_{n+1}}}\left|\begin{array}{cccc}
f_{0,0} & f_{0,1} & \ldots & f_{0, n} \\
f_{1,0} & f_{1,1} & \ldots & f_{1, n} \\
\vdots & \vdots & \ddots & \vdots \\
f_{n-1,0} & f_{n-1,1} & \ldots & f_{n-1, n} \\
1 & z & \ldots & z^{n}
\end{array}\right|
$$

and

$$
\hat{\phi}_{n}\left(z^{-1}\right)=\frac{1}{\sqrt{D_{n} D_{n+1}}}\left|\begin{array}{ccccc}
f_{0,0} & f_{0,1} & \cdots & f_{0, n-1} & 1 \\
f_{1,0} & f_{1,1} & \cdots & f_{1, n-1} & z^{-1} \\
\vdots & \vdots & \ddots & \vdots & \vdots \\
f_{n, 0} & f_{n, 1} & \cdots & f_{n, n-1} & z^{-n}
\end{array}\right|
$$

where $f_{j, k}=f_{j-k}$. Evaluating these determinants along the bottom row and far right column, respectively, immediately gives the leading coefficient for both to be

$$
\chi_{n}=\sqrt{\frac{D_{n}}{D_{n+1}}} .
$$

Consider the symbol $f_{t}$ defined in (38). It follows from Theorem 1 that $D_{n}\left(f_{t}\right) \neq 0$ for $n \geq$ $n_{0}, n_{0}+1, \ldots$ with some $n_{0}$, and hence a system of orthonormal polynomials $\phi_{n}$ and $\hat{\phi}_{n}$ with respect to $f_{t}$ exist. Define a $2 \times 2$ matrix valued function $Y^{(n)}$ by

$$
Y^{(n)}(z)=Y(z)=\left(\begin{array}{cc}
\chi_{n}^{-1} \phi_{n}(z) & \chi_{n}^{-1} \int_{\mathbb{T}} \frac{\phi_{n}(\xi)}{\xi-z} \frac{f_{t}(\xi) d \xi}{2 \pi i \xi} \\
-\chi_{n-1} z^{n-1} \hat{\phi}_{n-1}\left(z^{-1}\right) & -\chi_{n-1} \int_{\mathbb{T}} \frac{\hat{\phi}_{n-1}\left(\xi^{-1}\right)}{\xi-z} \frac{f_{t}(\xi) d \xi}{2 \pi i \xi}
\end{array}\right)
$$

for $n \geq n_{0}$. The matrix-valued function above is the unique solution to the following RiemannHilbert problem:

$R$-H problem for $Y$ (OPs with weight $f_{t}$ )

(Y1) $\quad Y: \mathbb{C} \backslash \mathbb{T} \rightarrow \mathbb{C}^{2 \times 2}$ is analytic.

(Y2) Let $z \in \mathbb{T} \backslash\left\{z_{1}\right\}$. $Y$ has continuous boundary values $Y_{+}(z)$ as $z$ approaches the unit circle from the inside, and $Y_{-}(z)$ from the outside, related by the jump condition

$$
Y_{+}(z)=Y_{-}(z)\left(\begin{array}{cc}
1 & z^{-n} f_{t}(z) \\
0 & 1
\end{array}\right), \quad z \in \mathbb{T} .
$$

(Y3) $Y(z)$ has the following asymptotic behavior at ininity:

$$
Y(z)=(I+\mathcal{O}(1 / z))\left(\begin{array}{cc}
z^{n} & 0 \\
0 & z^{-n}
\end{array}\right) \quad \text { as } z \rightarrow \infty .
$$

$$
\text { As } z \rightarrow z_{1}, z \in \mathbb{C} \backslash \mathbb{T}
$$

$$
Y(z)=\left(\begin{array}{ll}
\mathcal{O}(1) & \mathcal{O}(1)+\mathcal{O}\left(\left|z-z_{1}\right|^{2 \alpha_{1}}\right) \\
\mathcal{O}(1) & \mathcal{O}(1)+\mathcal{O}\left(\left|z-z_{1}\right|^{2 \alpha_{1}}\right)
\end{array}\right), \quad \text { if } \alpha_{1} \neq 0
$$

and

$$
Y(z)=\left(\begin{array}{ll}
\mathcal{O}(1) & \mathcal{O}(1)+\mathcal{O}\left(\log \left|z-z_{1}\right|\right) \\
\mathcal{O}(1) & \mathcal{O}(1)+\mathcal{O}\left(\log \left|z-z_{1}\right|\right)
\end{array}\right), \quad \text { if } \alpha_{1}=0, \beta_{1} \neq 0
$$




\section{Differential iDEntity}

In this section, we derive a differential identity that links the Toeplitz determinants $D_{n}\left(f_{t}\right)$ to the Riemann-Hilbert problem for polynomials orthogonal with respect to the weight $f_{t}$ defined in (38). The differential identity is analogous to the one in [3], but with the weight of the polynomials replaced by $f_{t}$. In [3] the identity was obtained using integral and integrable Fredholm operators, while here we use orthogonal polynomials instead.

Lemma 7. Let $t>0$ and $n \in \mathbb{N}$. Suppose that the Riemann-Hilbert problem for $Y(z ; n, t)$ in Section 5 with $f_{t}(z)$ given by (38) is solvable. Then the following differential identity holds:

$$
\frac{\partial}{\partial t} \log D_{n}\left(f_{t}\right)=-\left(\alpha_{0}+\beta_{0}\right) e^{t}\left(Y^{-1} \frac{d Y}{d z}\right)_{22}\left(e^{t}\right)+\left(\alpha_{0}-\beta_{0}\right) e^{-t}\left(Y^{-1} \frac{d Y}{d z}\right)_{22}\left(e^{-t}\right),
$$

where $\left(Y^{-1} \frac{d Y}{d z}\right)_{22}(\xi)$ denotes the 22 entry of the matrix obtained by multiplying the two matrices $Y^{-1}(z)$ and $\frac{d Y}{d z}(z)$ (each entry of $Y$ is differentiated with respect to $z$ ) together, evaluated at $z=\xi$.

Proof. As observed in [4, solvability of the Riemann-Hilbert problem for $Y(z ; n, t)$ is equivalent to $D_{j} \neq 0$ for $j=n-1, n, n+1$, which implies the solvability in a neighborhood of $t$. Also, as stated in [4], the following identity (which was derived in [8] under the assumption that $D_{k} \neq 0$ for any $k \in \mathbb{N}$ ) can be derived under the weaker assumption that $D_{j} \neq 0$ for $j=n-1, n, n+1$ :

$$
\frac{\partial}{\partial t} \log D_{n}\left(f_{t}(z)\right)=2 n \frac{\frac{\partial \chi_{n}}{\partial t}}{\chi_{n}}+\frac{1}{2 \pi i} \int_{\mathbb{T}} \frac{\partial}{\partial t}\left(\phi_{n}(z) \frac{d \hat{\phi}_{n}\left(z^{-1}\right)}{d z}-\hat{\phi}_{n}\left(z^{-1}\right) \frac{d \phi_{n}(z)}{d z}\right) f_{t}(z) d z .
$$

Using the Leibniz rule,

$$
\frac{\partial}{\partial t} \int_{\mathbb{T}} F(z) f_{t}(z) d z=\int_{\mathbb{T}} \frac{\partial}{\partial t}\left(F(z) f_{t}(z)\right) d z
$$

provided that the functions $F(z)$ and $\frac{\partial F(z)}{\partial t}$ are analytic in a neighborhood of $\mathbb{T}$. By the product rule,

$$
\int_{\mathbb{T}} \frac{\partial F(z)}{\partial t} f_{t}(z) d z=\frac{\partial}{\partial t} \int_{\mathbb{T}} F(z) f_{t}(z) d z-\int_{\mathbb{T}} F(z) \frac{\partial f_{t}(z)}{\partial t} d z .
$$

Letting $F(z)=\phi_{n}(z) \frac{d \hat{\phi}_{n}\left(z^{-1}\right)}{d z}-\hat{\phi}_{n}\left(z^{-1}\right) \frac{d \phi_{n}(z)}{d z}$ and noting that by linearity, and orthogonality conditions 50 ,

$$
\frac{\partial}{\partial t}\left(\frac{1}{2 \pi i} \int_{\mathbb{T}}\left(\phi_{n}(z) \frac{d \hat{\phi}_{n}\left(z^{-1}\right)}{d z}-\hat{\phi}_{n}\left(z^{-1}\right) \frac{d \phi_{n}(z)}{d z}\right) z f_{t}(z) \frac{d z}{z}\right)=\frac{\partial}{\partial t}(-2 n)=0,
$$

we now have,

$$
\frac{\partial}{\partial t} \log D_{n}\left(f_{t}(z)\right)=2 n \frac{\frac{\partial \chi_{n}}{\partial t}}{\chi_{n}}-\frac{1}{2 \pi i} \int_{\mathbb{T}}\left(\phi_{n}(z) \frac{d \hat{\phi}_{n}\left(z^{-1}\right)}{d z}-\hat{\phi}_{n}\left(z^{-1}\right) \frac{d \phi_{n}(z)}{d z}\right) \frac{\partial f_{t}(z)}{\partial t} d z .
$$

Computing the derivative gives,

$$
\frac{\partial f_{t}(z)}{\partial t}=\left(-\frac{\alpha_{0}+\beta_{0}}{z-e^{t}} e^{t}+\frac{\alpha_{0}-\beta_{0}}{z-e^{-t}} e^{-t}\right) f_{t}(z),
$$

thus we obtain,

$$
\frac{\partial}{\partial t} \log D_{n}\left(f_{t}(z)\right)=2 n \frac{\frac{\partial \chi_{n}}{\partial t}}{\chi_{n}}+\left(\alpha_{0}+\beta_{0}\right) \frac{e^{t}}{2 \pi i} \int_{\mathbb{T}} \frac{F(z) f_{t}(z)}{z-e^{t}} d z-\left(\alpha_{0}-\beta_{0}\right) \frac{e^{-t}}{2 \pi i} \int_{\mathbb{T}} \frac{F(z) f_{t}(z)}{z-e^{-t}} d z .
$$


We are left with evaluating the following integral,

$$
\frac{1}{2 \pi i} \int_{\mathbb{T}} \frac{F(z) f_{t}(z)}{z-\xi} d z=I_{1}-I_{2}
$$

where $\xi=e^{t}$ or $e^{-t}$, and,

$$
I_{1}=\frac{1}{2 \pi i} \int_{\mathbb{T}} \frac{\phi_{n}(z) \frac{d \hat{\phi}_{n}\left(z^{-1}\right)}{d z}}{z-\xi} f_{t}(z) d z \quad \text { and } \quad I_{2}=\frac{1}{2 \pi i} \int_{\mathbb{T}} \frac{\hat{\phi}_{n}\left(z^{-1}\right) \frac{d \phi_{n}(z)}{d z}}{z-\xi} f_{t}(z) d z .
$$

Note first that for any polynomial of degree $n \in \mathbb{Z}_{+}, p_{n}(z)=a_{n} z^{n}+a_{n-1} z^{n-1}+\ldots+a_{1} z+a_{0}$,

$$
\begin{aligned}
\frac{p_{n}(z)-p_{n}(\xi)}{z-\xi} & =\frac{a_{n}\left(z^{n}-\xi^{n}\right)+a_{n-1}\left(z^{n-1}-\xi^{n-1}\right)+\ldots+a_{1}(z-\xi)}{z-\xi} \\
& =\frac{a_{n}(z-\xi)\left(z^{n-1}+\xi^{n-1}\right)-z \xi^{n-1}+\xi z^{n-1}+\ldots}{z-\xi}=a_{n} z^{n-1}+\mathcal{O}\left(z^{n-2}\right),
\end{aligned}
$$

which also holds for $n \in \mathbb{Z}_{-}$by substituting $z \mapsto z^{-1}$ and $\xi \mapsto \xi^{-1}$.

Now, starting with $I_{1}$, adding and subtracting $\left.\frac{d \hat{\phi}_{n}\left(z^{-1}\right)}{d z}\right|_{z=\xi}$ in the numerator and using orthogonality 50 gives,

$$
\begin{aligned}
I_{1} & =\frac{1}{2 \pi i} \int-\phi_{n}(z) \frac{\left(\frac{d \hat{\phi}_{n}\left(z^{-1}\right)}{d z}-\left.\frac{d \hat{\phi}_{n}\left(z^{-1}\right)}{d z}\right|_{z=\xi}\right)}{\frac{1}{z}-\frac{1}{\xi}}(z \xi)^{-1} f_{t}(z) d z+\frac{1}{2 \pi i} \int \frac{\left.\phi_{n}(z) \frac{d \hat{\phi}_{n}\left(z^{-1}\right)}{d z}\right|_{z=\xi}}{z-\xi} f_{t}(z) d z \\
& =n \xi^{-1}+\left.\frac{d \hat{\phi}_{n}\left(z^{-1}\right)}{d z}\right|_{z=\xi} \frac{1}{2 \pi i} \int \phi_{n}(z)\left(z^{n-1}+\ldots\right) \frac{f_{t}(z)}{z^{n}} d z+\left.\frac{d \hat{\phi}_{n}\left(z^{-1}\right)}{d z}\right|_{z=\xi} \frac{1}{2 \pi i} \int \frac{\phi_{n}(z)}{z-\xi} \xi^{n} \frac{f_{t}(z)}{z^{n}} d z,
\end{aligned}
$$

and by comparing with the entries of the R-H problem (55),

$$
I_{1}=n \xi^{-1}+\left.\xi^{n} \chi_{n} \frac{d \hat{\phi}_{n}\left(z^{-1}\right)}{d z}\right|_{z=\xi} Y_{12}(\xi)
$$

We now look at $I_{2}$, proceeding in the same way as before we obtain

$$
I_{2}=\frac{1}{2 \pi i} \int \hat{\phi}_{n}\left(z^{-1}\right)\left(n \chi_{n} z^{n-1}+\ldots\right) f_{t}(z) \frac{d z}{z}+\left.\frac{d \phi_{n}(z)}{d z}\right|_{z=\xi} \frac{1}{2 \pi i} \int \frac{\hat{\phi}_{n}\left(z^{-1}\right)}{z-\xi} f_{t}(z) d z .
$$

By orthogonality in the first term and by using the following recurrence relation:

$$
\chi_{n} \hat{\phi}_{n}\left(z^{-1}\right)=\chi_{n-1} z^{-1} \hat{\phi}_{n-1}\left(z^{-1}\right)+\hat{\phi}_{n}(0) z^{-n} \phi_{n}(z)
$$

in the second, we obtain

$$
I_{2}=-\left.\frac{d Y_{11}(z)}{d z}\right|_{z=\xi} Y_{22}(\xi)+\left.\frac{d \phi_{n}(z)}{d z}\right|_{z-\xi} \hat{\phi}_{n}(0) Y_{12}(\xi)
$$

We now combine the two results. Using the same recurrence relation as above for $\hat{\phi}_{n}\left(z^{-1}\right)$ and collecting the $Y_{12}(\xi)$ terms gives,

$$
\begin{aligned}
& I_{1}-I_{2}=n \xi^{-1}+\left.\frac{d Y_{11}(z)}{d z}\right|_{z=\xi} Y_{22}(\xi) \\
& +\left\{\xi^{-1}\left(-\chi_{n-1} \xi^{n-1} \hat{\phi}_{n-1}\left(\xi^{-1}\right)\right)+\left.\chi_{n-1} \xi^{n-1} \frac{d \hat{\phi}_{n-1}\left(z^{-1}\right)}{d z}\right|_{z=\xi}-n \hat{\phi}_{n}(0) \xi^{-1} \phi_{n}(\xi)\right\} Y_{12}(\xi) .
\end{aligned}
$$


Adding and subtracting $\chi_{n-1}(n-1) \xi^{n-2} \hat{\phi}_{n-1}\left(\xi^{-1}\right)$ and noting that

$$
\frac{d Y_{21}(z)}{d z}=-\chi_{n-1}(n-1) z^{n-2} \hat{\phi}_{n-1}\left(z^{-1}\right)-\chi_{n-1}\left(z^{n-1}\right) \frac{d \hat{\phi}_{n-1}\left(z^{-1}\right)}{d z},
$$

we get, using (55),

$$
I_{1}-I_{2}=n \xi^{-1}+\left.\frac{d Y_{11}(z)}{d z}\right|_{z=\xi} Y_{22}(\xi)+\left\{n \xi^{-1} Y_{21}(\xi)-\left.\frac{d Y_{21}(z)}{d z}\right|_{z=\xi}-n \hat{\phi}_{n}(0) \xi^{-1} \chi_{n} Y_{11}(\xi)\right\} Y_{12}(\xi) .
$$

Next, we look at the term $\frac{2 n \frac{\partial \chi_{n}}{\partial t}}{\chi_{n}}$ in 66 . Noting that

$$
\frac{1}{2 \pi i} \int \frac{\partial \phi_{n}(z)}{d t} \hat{\phi}_{n}\left(z^{-1}\right) f_{t}(z) \frac{d z}{z}=\frac{\frac{\partial \chi_{n}}{\partial t}}{\chi_{n}}=\frac{1}{2 \pi i} \int \frac{\partial \hat{\phi}_{n}\left(z^{-1}\right)}{d t} \phi(z) f_{t}(z) \frac{d z}{z}
$$

we have

$$
2 \frac{\frac{\partial \chi_{n}}{\partial t}}{\chi_{n}}=\frac{1}{2 \pi i} \int \frac{\partial}{\partial t}\left(\phi_{n}(z) \hat{\phi}_{n}\left(z^{-1}\right)\right) f_{t}(z) \frac{d z}{z} .
$$

Using (63) and that $\frac{\partial}{\partial t}\left[\frac{1}{2 \pi i} \int_{\mathbb{T}} \hat{\phi}_{n}\left(z^{-1}\right) \phi_{n}(z) f_{t}(z) \frac{d z}{z}\right]=0$,

$$
\begin{aligned}
2 \frac{\frac{\partial \chi_{n}}{\partial t}}{\chi_{n}} & =-\frac{1}{2 \pi i} \int \phi_{n}(z) \hat{\phi}_{n}\left(z^{-1}\right) \frac{\partial f_{t}(z)}{d t} \frac{d z}{z} \\
& =\left(\alpha_{0}+\beta_{0}\right) e^{t} \frac{1}{2 \pi i} \int \frac{\phi_{n}(z) \hat{\phi}_{n}\left(z^{-1}\right) f_{t}(z)}{z-e^{t}} \frac{d z}{z}-\left(\alpha_{0}-\beta_{0}\right) e^{-t} \frac{1}{2 \pi i} \int \frac{\phi_{n}(z) \hat{\phi}_{n}\left(z^{-1}\right) f_{t}(z)}{z-e^{-t}} \frac{d z}{z} .
\end{aligned}
$$

We now evaluate $\frac{1}{2 \pi i} \int \frac{\phi_{n}(z) \hat{\phi}_{n}\left(z^{-1}\right) f_{t}(z)}{z-\xi} \frac{d z}{z}$, where $\xi=e^{ \pm t}$. Using the same ideas as before, adding and subtracting $\hat{\phi}_{n}\left(\xi^{-1}\right)$ in the numerator, using (67), adding and subtracting $\xi^{n-1}$, using orthogonality and (68) gives

$$
\frac{1}{2 \pi i} \int \phi_{n}(z) \frac{\hat{\phi}_{n}\left(z^{-1}\right)}{z-\xi} f_{t}(z) \frac{d z}{z}=-\xi^{-1}-\xi^{-1} Y_{21}(\xi) Y_{12}(\xi)+\hat{\phi}_{n}(0) \chi_{n} \xi^{-1} Y_{11}(\xi) Y_{12}(\xi) .
$$

Finally, we combine all of the results and after cancellations we obtain,

$$
\begin{aligned}
\frac{\partial}{\partial t} \log D_{n}(t)=- & \left(\alpha_{0}+\beta_{0}\right) e^{t}\left(-\left.\frac{d Y_{11}(z)}{d z}\right|_{z=e^{t}} Y_{22}\left(e^{t}\right)+\left.\frac{d Y_{21}(z)}{d z}\right|_{z=e^{t}} Y_{12}\left(e^{t}\right)\right) \\
& +\left(\alpha_{0}-\beta_{0}\right) e^{-t}\left(-\left.\frac{d Y_{11}(z)}{d z}\right|_{z=e^{-t}} Y_{22}\left(e^{-t}\right)+\left.\frac{d Y_{21}(z)}{d z}\right|_{z=e^{-t}} Y_{12}\left(e^{-t}\right)\right) .
\end{aligned}
$$

We thus have that,

$$
\frac{\partial}{\partial t} \log D_{n}(t)=-\left(\alpha_{0}+\beta_{0}\right) e^{t}\left(-\frac{d Y^{-1}}{d z} Y\right)_{22}\left(e^{t}\right)+\left(\alpha_{0}-\beta_{0}\right) e^{-t}\left(-\frac{d Y^{-1}}{d z} Y\right)_{22}\left(e^{-t}\right),
$$

which is exactly 60 , noting that $0=\frac{d}{d z}\left(Y^{-1} Y\right)=Y^{-1} \frac{d Y}{d z}+\frac{d Y^{-1}}{d z} Y$.

\section{Asymptotic analysis of the R-H PRoblem}

In this section we will solve asymptotically the Riemann-Hilbert problem which was posed in Section 5. In order to evaluate the asymptotics for the solution, the problem needs to undergo a series of reversible transformations. We will be using the method of nonlinear steepest descent, which was introduced by Deift and Zhou in the 1990s, see [10]. For an illustrative example of this method, see [5], which proves the strong Szegö limit theorem. We proceed with the analysis in a similar way to that in $[3,4,6,8]$. 
7.1. Normalization. The Riemann-Hilbert problem for $Y$ lacks the right behavior at infinity. To normalize the problem at infinity, we define a new function $T$ by

$$
T(z)= \begin{cases}Y(z) z^{-k \sigma_{3}}, & \text { as }|z|>1 \\ Y(z), & \text { as }|z|<1\end{cases}
$$

where,

$$
\sigma_{3}=\left(\begin{array}{cc}
1 & 0 \\
0 & -1
\end{array}\right)
$$

is one of the Pauli matrices.

We now have two equivalent Riemann-Hilbert problems; in particular, if $Y$ solves the RiemannHilbert problem for orthogonal polynomials (55), then $T$ solves the following Riemann-Hilbert problem:

(T1) $\quad T: \mathbb{C} \backslash \mathbb{T} \rightarrow \mathbb{C}^{2 \times 2}$ is analytic.

(T2) $\quad T_{+}(z)=T_{-}(z) J_{T}(z)$ for $z \in \mathbb{T}$, where

$$
J_{T}(z)=\left(\begin{array}{cc}
z^{k} & f_{t}(z) \\
0 & z^{-k}
\end{array}\right)
$$

(T3) $T(z)$ has the following asymptotic behavior at ininity,

$$
T(z)=I+\mathcal{O}(1 / z) \quad \text { as } z \rightarrow \infty .
$$

(T4) The asymptotic formulae close to point $z_{1}$ is the same as in the problem for $Y(z)$.

The two problems are equivalent in the sense that we can obtain a solution of one problem, using the solution to the other, and vice versa, via simple algebraic manipulation.

Notice that the diagonal entries of the jump matrix $J_{T}$ in (T2) above oscillate rapidly on the unit circle for large $k$. The next transformation will turn this oscillatory behavior on the unit circle into exponential decay on a deformed contour.

7.2. Opening of the lenses. First we factorize the jump matrix $J_{T}$ as follows:

$$
J_{T}(z)=J_{1}(z) J_{N}(z) J_{2}(z)=\left(\begin{array}{cc}
1 & 0 \\
z^{-k} f_{t}(z)^{-1} & 1
\end{array}\right)\left(\begin{array}{cc}
0 & f_{t}(z) \\
-f_{t}(z)^{-1} & 0
\end{array}\right)\left(\begin{array}{cc}
1 & 0 \\
z^{k} f_{t}(z)^{-1} & 1
\end{array}\right) .
$$

The initial contour $\mathbb{T}$ is now deformed as shown in Figure 5 . The matrix-valued functions $J_{1}$ and $J_{2}$ are both invertible, and have analytic continuations (which are also invertible) to the outside and inside of the unit disk, respectively - intersected with the annulus where the function $f_{t}$ is analytic. We also note the desired decay of the off-diagonal terms in the two matrices, in the respective regions of analytic continuation.

We use $J_{1}$ and $J_{2}$ to deform the problem for $T$ by defining a new function $S$ as follows:

$$
S(z)= \begin{cases}T(z), & \text { for } z \in \Omega_{1} \cup \Omega_{4}, \\ T(z) J_{1}(z), & \text { for } z \in \Omega_{2} \cup \Omega_{2}^{\prime}, \\ T(z) J_{2}^{-1}(z), & \text { for } z \in \Omega_{3} \cup \Omega_{3}^{\prime} .\end{cases}
$$

The function $S$ solves the following R-H problem:

$S$ is analytic in $\mathbb{C} \backslash \Sigma$, where $\Sigma=\bigcup_{j=0}^{1}\left(\Sigma_{j} \cup \Sigma_{j}^{\prime} \cup \Sigma_{j}^{\prime \prime}\right)$.

The boundary values are related by the following jump conditions:

$$
\begin{gathered}
S_{+}(z)=S_{-}(z) J_{1}(z), \quad z \in \Sigma_{0} \cup \Sigma_{1}, \\
S_{+}(z)=S_{-}(z) J_{N}(z), \quad z \in \Sigma_{0}^{\prime} \cup \Sigma_{1}^{\prime}, \\
S_{+}(z)=S_{-}(z) J_{2}(z), \quad z \in \Sigma_{0}^{\prime \prime} \cup \Sigma_{1}^{\prime \prime} . \\
S(z)=T(z)=I+\mathcal{O}(1 / z) \text { as } z \rightarrow \infty .
\end{gathered}
$$




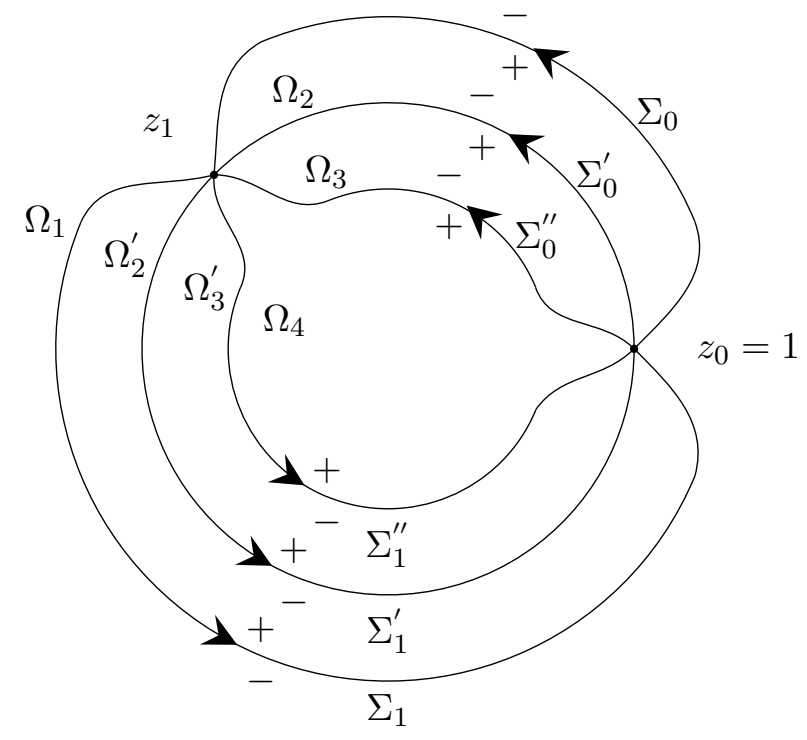

Figure 5. Deformed contour $\Sigma=\cup_{j=0}^{1}\left(\Sigma_{j} \cup \Sigma_{j}^{\prime} \cup \Sigma_{j}^{\prime \prime}\right)$, and regions $\Omega_{j}, \Omega_{j}^{\prime}$ for the Riemann-Hilbert problem for $S$. Note that $\mathbb{T}=\Sigma_{0}^{\prime} \cup \Sigma_{1}^{\prime}$.

As $z \rightarrow z_{1}, z \in \mathbb{C} \backslash \mathbb{T}$, outside the lense (i.e. in $\Omega_{1} \cup \Omega_{4}$ )

$$
S(z)=\left(\begin{array}{ll}
\mathcal{O}(1) & \mathcal{O}\left(\left|z-z_{1}\right|^{2 \alpha_{1}}\right)+\mathcal{O}(1) \\
\mathcal{O}(1) & \mathcal{O}\left(\left|z-z_{1}\right|^{2 \alpha_{1}}\right)+\mathcal{O}(1)
\end{array}\right) \quad \alpha_{1} \neq 0
$$

and

$$
S(z)=\left(\begin{array}{ll}
\mathcal{O}(1) & \mathcal{O}\left(\log \left|z-z_{1}\right|\right) \\
\mathcal{O}(1) & \mathcal{O}\left(\log \left|z-z_{1}\right|\right)
\end{array}\right) \quad \alpha_{1}=0, \beta_{1} \neq 0
$$

The asymptotic behavior in $\Omega_{2} \cup \Omega_{2}^{\prime}$ and $\Omega_{3} \cup \Omega_{3}^{\prime}$ is given by applying jump conditions to the expressions (76) and (77).

The new Riemann-Hilbert problem $(S, \Sigma)$ is called a deformation of the problem $(T, \mathbb{T})$ and can be compared to the deformation of a contour in the method of evaluating a classical steepest descent problem in complex analysis.

Let us encircle the two points $z_{0}=1$ and $z_{1}$ by $\varepsilon$-small disks

$$
U_{z_{j}}=\left\{z:\left|z-z_{j}\right|<\varepsilon\right\}, \quad j=0,1 .
$$

We shall construct a global parametrix dealing with the jump condition over the unit circle and two local parametrices around the points of intersection $z_{0}$ and $z_{1}$.

7.3. Global Parametrix. We consider a Riemann-Hilbert problem for $N$, ignoring the contours $\bigcup_{j=0}^{1}\left(\Sigma_{j} \cup \Sigma_{j}^{\prime \prime}\right)$ and the neighborhoods $\bigcup_{j=0}^{1} U_{z_{j}}$. The model problem is given by

(N1) $\quad N: \mathbb{C} \backslash \mathbb{T} \rightarrow \mathbb{C}^{2 \times 2}$ is analytic,

(N2) $\quad N_{+}(z)=N_{-}(z) J_{N}(z)$, for $z \in \mathbb{T}$,

(N3) $\quad N(z)=I+\mathcal{O}(1 / z)$, as $z \rightarrow \infty$. 


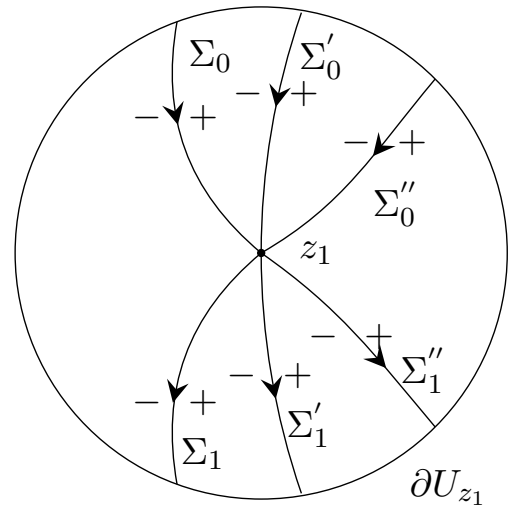

(A) The neighborhood $U_{z_{1}}$

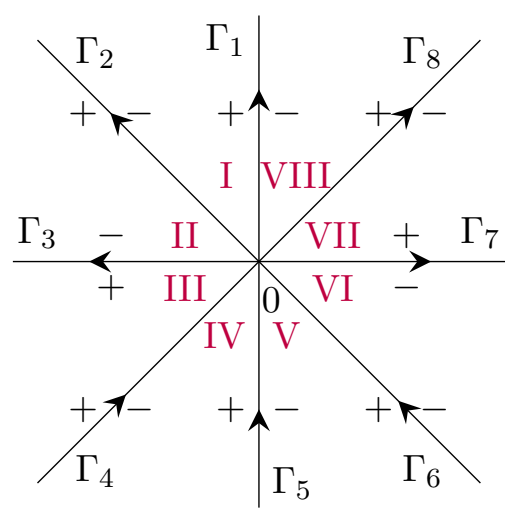

(B) $U_{z_{1}}$ under the $\zeta$ transformation (called $\left.\zeta\left(U_{z_{1}}\right)\right)$. Contour for the $\Psi_{1} \mathrm{R}-\mathrm{H}$ problem.

\section{FiguRE 6}

Similarly to [3, 6], the problem is explicitly solvable and the solution is given in terms of the Szegö function

$$
D(z)=\exp \left\{\frac{1}{2 \pi i} \int_{\mathbb{T}} \frac{\log f_{t}(s)}{s-z} d s\right\}
$$

Observe that

$$
N(z)= \begin{cases}D(z)^{\sigma_{3}}\left(\begin{array}{cc}
0 & 1 \\
-1 & 0
\end{array}\right), & \text { for }|z|<1 \\
D(z)^{\sigma_{3}}, & \text { for }|z|>1\end{cases}
$$

By evaluating the integral in $(79)$, we can compute the following explicit formula for the Szegö function $D$ :

$$
D(z)= \begin{cases}\left(\frac{z-z_{1}}{z_{1} e^{i \pi}}\right)^{\alpha_{1}+\beta_{1}}\left(z-e^{t}\right)^{\alpha_{0}+\beta_{0}} e^{-i \pi\left(\alpha_{0}+\beta_{0}\right)} \exp \left\{\sum_{k=0}^{\infty} V_{k} z^{k}\right\}, & \text { for }|z|<1, \\ \left(\frac{z-z_{1}}{z}\right)^{-\alpha_{1}+\beta_{1}}\left(z-e^{-t}\right)^{-\alpha_{0}+\beta_{0}} z^{\alpha_{0}-\beta_{0}} \exp \left\{-\sum_{-\infty}^{-1} V_{k} z^{k}\right\}, & \text { for }|z|>1 .\end{cases}
$$

We now go back to the Riemann-Hilbert problem for $S$ where we have opened the lens and created a contour which possesses intersections. We will look at each intersection separately. On their own, the local parametrices at $z_{0}$ and $z_{1}$ are the same as in [6] and [3], respectively.

7.4. Parametrix at point $z_{1}$. In this section, we are basing the analysis on the works of Deift, Its and Krasovsky in [6, 8]. We first construct the parametrix $P_{z_{1}}(z)$ in the neighborhood $U_{z_{1}}$, see (78). We again look for a sectionally analytic matrix-valued function, this time in the neighborhood $U_{z_{1}}$ as opposed to the whole complex plane. This function will have the same jump conditions as $S(z)$, again only restricted to the intersection $\Sigma \cap U_{z_{1}}$. It will also have the same behavior as $z \rightarrow z_{1}$ as the function $S,(76),(77)$. However, instead of being normalized at infinity, the new function will satisfy the matching condition

$$
P_{z_{1}}(z) N^{-1}(z)=I+o(1) \quad \text { as } n \rightarrow \infty .
$$

Consider the following transformation (which is where the $n$ appears),

$$
\zeta=n \log \frac{z}{z_{1}},
$$


where $\log x>0$ for $x>0$, and the logarithm has a cut on the negative half of the real axis. This transformation maps the neighborhood $U_{z_{1}}$, which can be seen in Figure 6a, into a neighborhood of zero in the $\zeta$-plane, Figure $6 \mathrm{~b}$. We choose the form of the $\Sigma \cap U_{z_{1}}$ to give straight lines under the transformation. The function $\zeta$ is analytic and bijective, it takes an arc of the unit circle (recall that $\Sigma_{0}^{\prime} \cup \Sigma_{1}^{\prime}=\mathbb{T}$ ) to an interval of the imaginary axis (see Figure 66. The inside of the unit circle corresponds to values of $\zeta$ in the sectors I, II, III, IV, whereas the outside corresponds to $\zeta \in \mathrm{V}, \mathrm{VI}, \mathrm{VII}, \mathrm{VIII}$. The pre-image of the rays $\Gamma_{3}$ and $\Gamma_{7}$ is added to the contour $\Sigma \cap U_{z_{1}}$ to deal with non-analyticity of $\left|z-z_{1}\right|^{\alpha_{j}}$, which we discuss next. As in [6, (4.13)], define

$$
h_{\alpha_{1}}(z)=\left|z-z_{1}\right|^{\alpha_{1}}=\left(z-z_{1}\right)^{\alpha_{1} / 2}\left(z^{-1}-z_{1}^{-1}\right)^{\alpha_{1} / 2}=\frac{\left(z-z_{1}\right)^{\alpha_{1}}}{\left(z z_{1} e^{i l_{1}}\right)^{\alpha_{1} / 2}},
$$

where $z=e^{i \theta}, z_{1}=e^{i \theta_{1}}, 0 \leq \theta<2 \pi$ and $\theta_{1} \neq 0$. We fix the cut of $\left(z-z_{1}\right)^{\alpha}$ going along the ray $\arg z=\arg z_{1}$ from $z_{1}$ to infinity, and we fix the branch by the condition that on the line from $z_{1}$ to the right parallel to the real axis, $\arg \left(z-z_{1}\right)=2 \pi$. For $z^{\alpha_{1} / 2}$ in the denominator, $0<\arg z<2 \pi$. We write

$$
\left(z-z_{1}\right)^{\alpha_{1}}=\exp \left\{\alpha_{1} \log \left(z-z_{1}\right)\right\}=\exp \left\{\alpha_{1} \log \left|z-z_{1}\right|+\alpha_{1} i \arg \left(z-z_{1}\right)\right\}
$$

and

$$
\left(z z_{1} e^{i l_{1}}\right)^{-\alpha_{1} / 2}=\exp \left\{-i \frac{\alpha_{1}}{2}\left(\theta+\theta_{1}+l_{1}\right)\right\}
$$

Thus,

$$
\frac{\left(z-z_{1}\right)^{\alpha_{1}}}{\left(z z_{1} e^{i l_{1}}\right)^{\alpha_{1} / 2}}=\left|z-z_{1}\right|^{\alpha_{1}} \exp \left\{i \alpha_{1}\left(\arg \left(z-z_{1}\right)-\frac{\theta}{2}-\frac{\theta_{1}}{2}-\frac{l_{1}}{2}\right)\right\},
$$

where we need the power of the exponential to be 0 . The values of $l_{1}$ are then given by

$$
l_{1}= \begin{cases}3 \pi, & 0<\theta<\theta_{1} \\ \pi, & \theta_{1}<\theta<2 \pi\end{cases}
$$

which can be seen by considering different triangles in the complex plane. Alternatively, we can write

$$
\frac{z-z_{1}}{\left(z z_{1} e^{i l_{1}}\right)^{1 / 2}}=\exp \left\{i\left(\frac{\pi-l_{1}}{2}+\pi k\right)\right\} \mp\left|z-z_{1}\right| \begin{cases}- & \text { for } 0 \leq \theta<\theta_{1} \\ + & \text { for } \theta>\theta_{1}\end{cases}
$$

which should equal $\left|z-z_{1}\right|$, and so

$$
\begin{aligned}
\exp \left\{i\left(\frac{\pi-l_{1}}{2}+\pi k\right)\right\} & = \begin{cases}-1=e^{i \pi} & \text { for } \theta<\theta_{1} \\
1=e^{0} & \text { for } \theta_{1}<\theta\end{cases} \\
l_{1} & =\left\{\begin{array}{lll}
(2 k-1) \pi & \bmod 4 \pi & \text { for } \theta<\theta_{1} \\
(2 k+1) \pi & \bmod 4 \pi & \text { for } \theta_{1}<\theta
\end{array}\right.
\end{aligned}
$$

We proceed in the same way and use the same notation as in [6, Section 4.2], with $j=1$. We define an auxiliary function $F_{1}$ (cf. [6. (4.15)]) by

$$
F_{1}(z)=\exp \left\{\frac{1}{2} \log a(z ; t)\right\}\left(\frac{z}{z_{1}}\right)^{\frac{\beta_{1}}{2}} h_{\alpha_{1}}(z) \times\left\{\begin{array}{ll}
e^{-i \pi \alpha_{1}}, & \zeta \in \mathrm{I}, \mathrm{II}, \mathrm{V}, \mathrm{VI}, \\
e^{i \pi \alpha}, & \zeta \in \mathrm{III}, \mathrm{IV}, \mathrm{VII}, \mathrm{VIII},
\end{array} z \in U_{z_{1}}\right.
$$


(for the sectors I-VIII, see Figure 6b). It can be verified that $F_{1}$ is analytic in the intersection of each quarter $\zeta$-plane with $\zeta\left(U_{z_{1}}\right)$ and has the following jumps:

$$
F_{1,+}(z)= \begin{cases}F_{1,-}(z) e^{-2 \pi i \alpha_{1}}, \quad \zeta \in \Gamma_{1}, \\ F_{1,-}(z) e^{2 \pi i \alpha_{1}}, \quad \zeta \in \Gamma_{5}, \\ F_{1,-}(z) e^{\pi i \alpha_{1}}, \quad \zeta \in \Gamma_{3} \cup \Gamma_{7} .\end{cases}
$$

It is easy to see, after considering the analytic continuation in (84) of $f_{t}$ off the arcs between singularities, and $a_{t}$, that

$$
\begin{aligned}
& F_{1}(z)^{2}=f_{t}(z) e^{-2 \pi i \alpha_{j}} g_{z_{1}, \beta_{1}}^{-1}(z), \quad \zeta \in \mathrm{I}, \mathrm{II}, \mathrm{V}, \mathrm{VI}, \\
& F_{1}(z)^{2}=f_{t}(z) e^{2 \pi i \alpha_{j}} g_{z_{1}, \beta_{1}}^{-1}(z), \quad \zeta \in \mathrm{III}, \mathrm{IV}, \mathrm{VII}, \mathrm{VIII} .
\end{aligned}
$$

We now look for $P_{z_{1}}(z)$ in the form

$$
P_{z_{1}}(z)=E(z) P^{(1)}(z) F_{1}(z)^{-\sigma_{3}} z^{ \pm n \sigma_{3} / 2}
$$

where the plus sign is taken for $|z|<1$ and minus for $|z|>1$, which corresponds to $\zeta \in$ I,II,III,IV, and $\zeta \in \mathrm{V}, \mathrm{VI}, \mathrm{VII}, \mathrm{VIII}$, respectively. The matrix $E$ is analytic and invertible in the neighborhood of $U_{z_{1}}$, and hence does not affect the jump and analyticity conditions and is chosen in order for $P_{z_{1}}$ to satisfy the matching condition on the boundary 82 .

Since $P_{z_{1}}$ has the same jump conditions as $S$, it is straightforward to verify that $P^{(1)}$ satisfies jump conditions with constant jump matrices. As in [6], we set

$$
P^{(1)}(z)=\Psi_{1}(\zeta)
$$

Then $\Psi_{1}(\zeta)$ satisfies the Riemann-Hilbert problem outlined in Section 7.5. We substitute asymptotics from 1119 into the condition on $E$ (see $(82)$ ),

$$
E(z) \Psi_{1}(\zeta) F_{1}(z)^{-\sigma_{3}} z^{ \pm n \sigma_{3} / 2} N^{-1}(z)=I+o(1),
$$

(with + for $|z|<1$, and - for $|z|>1$ ), to find that

$$
E(z)=N(z) \zeta^{\beta_{1} \sigma_{3}} F_{1}^{\sigma_{3}}(z) z_{1}^{-n \sigma_{3} / 2}\left(\begin{array}{cc}
e^{-i \pi\left(2 \beta_{1}+\alpha_{1}\right)} & 0 \\
0 & e^{i \pi\left(\beta_{1}+2 \alpha_{1}\right)}
\end{array}\right), \quad \text { for } \zeta \in \text { I,II. }
$$

The matrix $E$ for the remaining sectors can be computed using the relevant asymptotics which are obtained using (119), see [6. Equations (4.42)-(4.50)] for those details.

We next obtain expansions in $u=z-z_{1}$, as $u \rightarrow 0$, which are unique to our problem. These will be used later on to compute Laurent expansions for the asymptotic solution in the final R-H problem for $R$. From (83), 81), (90), (84), and using the factorization of $V(z)=$ $\exp \left\{\sum_{k=0}^{\infty} V_{k} z^{k}\right\} e^{V_{0}} \exp \left\{\sum_{k=0}^{\infty} V_{-k} z^{-k}\right\}$, we get

$$
\begin{gathered}
F_{1}(z)=\exp \left\{\log a\left(z_{1} ; t\right) / 2\right\} e^{-3 i \pi \alpha_{1} / 2} z_{1}^{-\alpha_{1}} u^{\alpha_{1}}(1+\mathcal{O}(u)), \quad \zeta \in \mathrm{I}, \\
D(z)=u^{\alpha_{1}+\beta_{1}} z_{1}^{-\left(\alpha_{1}+\beta_{1}\right)} e^{-i \pi\left(\alpha_{1}+\beta_{1}\right)}\left(z_{1}-e^{t}\right)^{\left(\alpha_{0}+\beta_{0}\right)} e^{-i \pi\left(\alpha_{0}+\beta_{0}\right)} \exp \left\{\sum_{k=0}^{\infty} V_{k} z_{1}^{k}\right\}(1+\mathcal{O}(u)), \\
\zeta(z)=n \log \frac{z}{z_{1}}=n \frac{u}{z_{1}}+\mathcal{O}\left(u^{2}\right)=n \frac{u}{z_{1}}(1+\mathcal{O}(u)) .
\end{gathered}
$$

Therefore,

$$
\left(\frac{D(z)}{\zeta^{\beta_{1}} F_{1}(z)}\right)^{2}=e^{V_{0}} \frac{\exp \left\{\sum_{k=1}^{\infty} V_{k} z_{1}^{k}\right\}}{\exp \left\{\sum_{k=1}^{\infty} V_{-k} z_{k}^{-k}\right\}}\left(1-e^{-t} z_{1}\right)^{\left(\alpha_{0}+\beta_{0}\right)}\left(1-e^{-t} z_{1}^{-1}\right)^{-\left(\alpha_{0}-\beta_{0}\right)}
$$




$$
\times e^{t\left(\alpha_{0}+\beta_{0}\right)} e^{i \pi\left(\alpha_{1}-2 \beta_{1}\right)} n^{-2 \beta_{1}}(1+\mathcal{O}(u)), \quad \zeta \in \mathrm{I} .
$$

From (96) it can be seen that $\operatorname{det} E(z)=e^{i \pi\left(\alpha_{1}-\beta_{1}\right)}$ (this holds for all sections I-VIII of the $\zeta$-plane in fact). We also note that $\operatorname{det} \Psi_{1}(\zeta)=e^{-i \pi\left(\alpha_{1}-\beta_{1}\right)}$. This can be seen from Liouville's theorem, the function det $\Psi_{1}(\zeta)$ has no jumps (verifying directly from the jump conditions in $\left(\Psi_{1} 2\right)$ above), the singularity at 0 is removable as $\operatorname{Re} \alpha_{1}>-1 / 2$ (by looking at the determinant of (113)) and the value of the function follows from computing the determinant of $(119)$. Those two facts, (93) and that $\operatorname{det} A^{\sigma_{3}}=1$ for any constant $A$, give $\operatorname{det} P_{z_{1}}(z)=1$, so $P_{z_{1}}(z)$ is invertible. Also observe that $S(z) P_{z_{1}}(z)^{-1}$ is analytic in $U_{z_{1}}$, which is needed for the final R-H problem in Section 7.8, By looking at (113), (114) and (76), (77), it can be seen that the singularity at $z_{1}$ is at most $\mathcal{O}\left(\left|z-z_{1}\right|^{2 \alpha_{1}}\right)$ or $\mathcal{O}\left(\log \left|z-z_{1}\right|\right)$, but by the construction of $P_{z_{1}}(z)$ (which as you recall, had the same jumps as $S(z)$ ), the matrix-valued function $S(z) P_{z_{1}}(z)^{-1}$ has no jumps in the neighborhood of $z_{1}$, so the singularity is removable.

We now compute the first correction term $\Delta_{1}(z)$ in the asymptotic series in inverse powers of $n$ of $(95)$,

$$
P_{z_{1}}(z) N^{-1}(z)=I+\Delta_{1}(z)+n^{-\operatorname{Re} \beta_{1} \sigma_{3}} \mathcal{O}\left(n^{-2}\right) n^{\operatorname{Re} \beta_{1} \sigma_{3}}
$$

A full series can be found by considering further terms in (119). We start by denoting by $E_{i j}$ and $\Psi_{1, i j}, i, j=1,2$, the matrix elements of $E(z)$ and the asymptotic expansion of $\Psi_{1}(\zeta)$ (see (119)). In what follows, we only compute using the values valid in sector I (equivalently, where appropriate $|z|<1$ ); the expression for the asymptotic series extends to the whole boundary $\partial U_{z_{1}}$ by analytic continuation by a consideration of other sectors. Multiplying out the matrices in (96) we get $E_{11}=E_{22}=0$, and

$$
\begin{gathered}
E_{12}=D(z) \zeta^{-\beta_{1}} F_{1}^{-1} z_{1}^{n / 2} e^{i \pi\left(\beta_{1}+2 \alpha_{1}\right)} \\
E_{21}=-E_{12}^{-1} e^{i \pi\left(\alpha_{1}-\beta_{1}\right)}
\end{gathered}
$$

Further, multiplying the matrices in 95 ,

$$
P_{z_{1}}(z) N^{-1}(z)=\left(\begin{array}{ll}
E_{12} \Psi_{1,22} F_{1} z^{-n / 2} D(z)^{-1} & -E_{12} \Psi_{1,21} F_{1}^{-1} z^{n / 2} D(z) \\
E_{21} \Psi_{1,12} F_{1} z^{-n / 2} D(z)^{-1} & -E_{21} \Psi_{1,11} F_{1}^{-1} z^{n / 2} D(z)
\end{array}\right),
$$

and substituting in 102, 103 and using 1190 for $\Psi_{1, i j}$ gives,

$$
\Delta_{1}(z)=\frac{1}{\zeta}\left(\begin{array}{cc}
-\left(\alpha_{1}^{2}-\beta_{1}^{2}\right) & z_{1}^{n} \frac{\Gamma\left(1+\alpha_{1}+\beta_{1}\right)}{\Gamma\left(\alpha_{1}-\beta_{1}\right)}\left(\frac{D(z)}{\zeta^{\beta} F_{1}}\right)^{2} e^{i \pi\left(2 \beta_{1}-\alpha_{1}\right)} \\
-z_{1}^{n} \frac{\Gamma\left(1+\alpha_{1}-\beta_{1}\right)}{\Gamma\left(\alpha_{1}+\beta_{1}\right)}\left(\frac{D(z)}{\zeta^{\beta} F_{1}}\right)^{-2} e^{-i \pi\left(2 \beta_{1}-\alpha_{1}\right)} & \left(\alpha_{1}^{2}-\beta_{1}^{2}\right)
\end{array}\right) .
$$

In particular,

$$
\begin{aligned}
\left(\Delta_{1}(z)\right)_{12}= & \frac{1}{\zeta} z_{1}^{n} e^{V_{0}}\left(1-z_{1} e^{-t}\right)^{\left(\alpha_{0}+\beta_{0}\right)}\left(1-e^{-t} z_{1}^{-1}\right)^{-\left(\alpha_{0}-\beta_{0}\right)} \exp \left\{\sum_{k=1}^{\infty} V_{k} z_{1}^{k}\right\} \exp \left\{-\sum_{k=1}^{\infty} V_{-k} z_{k}^{-k}\right\} \\
& \times e^{t\left(\alpha_{0}-\beta_{0}\right)} n^{-2 \beta_{1}} \frac{\Gamma\left(1+\alpha_{1}+\beta_{1}\right)}{\Gamma\left(\alpha_{1}-\beta_{1}\right)}(1+\mathcal{O}(u)) .
\end{aligned}
$$

We also note,

$$
\frac{1}{\zeta}=\frac{z_{1}}{n\left(z-z_{1}\right)}+\frac{1}{2 n}+\mathcal{O}\left(z-z_{1}\right), \quad z \rightarrow z_{1}
$$


7.5. Riemann-Hilbert Problem for $\Psi_{1}$. Let $\Psi_{1}(\zeta)$ be the solution of the following RiemannHilbert problem on the contour $\Gamma:=\cup_{k=1}^{8} \Gamma_{k}$ given in Figure $6 \mathrm{~b}$.

$\left(\Psi_{1} 1\right) \Psi_{1}: \mathbb{C} \backslash \Gamma \rightarrow \mathbb{C}^{2 \times 2}$ is analytic.

$\left(\Psi_{1} 2\right) \Psi_{1}$ satisfies the following jump conditions:

$$
\begin{gathered}
\Psi_{1,+}(\zeta)=\Psi_{1,-}(\zeta)\left(\begin{array}{cc}
0 & e^{-i \pi \beta_{1}} \\
-e^{i \pi \beta_{1}} & 0
\end{array}\right), \quad \text { for } \zeta \in \Gamma_{1}, \\
\Psi_{1,+}(\zeta)=\Psi_{1,-}(\zeta)\left(\begin{array}{cc}
0 & e^{i \pi \beta_{1}} \\
-e^{-i \pi \beta_{1}} & 0
\end{array}\right), \quad \text { for } \zeta \in \Gamma_{5}, \\
\Psi_{1,+}(\zeta)=\Psi_{1,-}(\zeta) e^{i \pi \alpha_{1} \sigma_{3}}, \quad \text { for } \zeta \in \Gamma_{3} \cup \Gamma_{7}, \\
\Psi_{1,+}(\zeta)=\Psi_{1,-}(\zeta)\left(\begin{array}{cc}
1 & 0 \\
e^{ \pm i \pi\left(\beta_{1}-2 \alpha_{1}\right)} & 1
\end{array}\right), \quad \text { with }\left\{\begin{array}{l}
+ \text { in the exponent for } \zeta \in \Gamma_{2}, \\
- \text { in the exponent for } \zeta \in \Gamma_{4},
\end{array}\right. \\
\Psi_{1,+}(\zeta)=\Psi_{1,-}(\zeta)\left(\begin{array}{cc}
1 & 0 \\
e^{ \pm i \pi\left(\beta_{1}+2 \alpha_{1}\right)} & 1
\end{array}\right), \quad \text { with }\left\{\begin{array}{l}
+ \text { in the exponent for } \zeta \in \Gamma_{8}, \\
- \text { in the exponent for } \zeta \in \Gamma_{6},
\end{array}\right.
\end{gathered}
$$

$\left(\Psi_{1} 3\right)$ As $\zeta \rightarrow 0, \zeta \in \mathbb{C} \backslash \Gamma$ outside the lenses (i.e. sectors II, III, VI, VII), for $\alpha_{1} \neq 0$,

$$
\Psi_{1}(\zeta)=\left(\begin{array}{ll}
\mathcal{O}\left(\zeta^{\alpha_{1}}\right) & \mathcal{O}\left(\zeta^{\alpha_{1}}\right)+\mathcal{O}\left(\zeta^{-\alpha_{1}}\right) \\
\mathcal{O}\left(\zeta^{\alpha_{1}}\right) & \mathcal{O}\left(\zeta^{\alpha_{1}}\right)+\mathcal{O}\left(\zeta^{-\alpha_{1}}\right)
\end{array}\right)
$$

and for $\alpha_{1}=0, \beta_{1} \neq 0$,

$$
\Psi_{1}(\zeta)=\left(\begin{array}{ll}
\mathcal{O}(1) & \mathcal{O}(\log |z|) \\
\mathcal{O}(1) & \mathcal{O}(\log |z|)
\end{array}\right)
$$

The behavior of $\Psi_{1}(\zeta)$ for $\zeta \rightarrow 0$ in other sectors can be computed using the appropriate jump conditions.

This problem was solved explicitly in [6, Section 4.2] and solutions found using confluent hypergeometric functions (see the appendix of [16]). Define a function $\psi(a, c ; z)$ as a unique solution of the confluent hypergeometric equation

$$
z w^{\prime \prime}+(c-a) w^{\prime}-a w=0,
$$

satisfying the following asymptotic condition

$$
\psi(a, c ; z) \sim z^{-a} \sum_{n=0}^{\infty}(-1)^{n} \frac{(a)_{n}(1+a-c)_{n}}{n ! z^{n}} \quad \text { as } \quad z \rightarrow \infty \text { and } \frac{-3 \pi}{2}<\arg z<\frac{3 \pi}{2},
$$

where

$$
(a)_{0}=1, \quad(a)_{n}=a(a+1) \ldots(a+n-1)=\frac{\Gamma(a+n)}{\Gamma(a)}, n \geq 1 .
$$

Theorem 8. [6, Proposition 4.1] Let $\alpha_{1} \pm \beta_{1} \neq-1,-2, \ldots$ Then a solution to the above RiemannHilbert problem $\left(\Psi_{1} 1\right)-\left(\Psi_{1} 3\right)$ for $\Psi_{1}, 0<\arg \zeta<2 \pi$, is given by the following matrix-valued function in sector $I$ :

$$
\begin{aligned}
\Psi_{1}(\zeta) & =\Psi_{1}^{(I)}(\zeta) \\
& =\left(\begin{array}{c}
\zeta^{\alpha_{1}} \psi\left(\alpha_{1}+\beta_{1}, 1+2 \alpha_{1}, \zeta\right) e^{i \pi\left(2 \beta_{1}+\alpha_{1}\right)} e^{-\zeta / 2} \\
-\zeta^{-\alpha_{1}} \psi\left(1-\alpha_{1}+\beta_{1}, 1-2 \alpha_{1}, \zeta\right) e^{i \pi\left(\beta_{1}-3 \alpha_{1}\right)} e^{-\zeta / 2} \frac{\Gamma\left(1+\alpha_{1}+\beta_{1}\right)}{\Gamma\left(\alpha_{1}-\beta_{1}\right)}
\end{array}\right.
\end{aligned}
$$




$$
\left.\begin{array}{c}
-\zeta^{\alpha_{1}} \psi\left(1+\alpha_{1}-\beta_{1}, 1+2 \alpha_{1}, e^{-i \pi} \zeta\right) e^{i \pi\left(\beta_{1}+\alpha_{1}\right)} e^{\zeta / 2} \frac{\Gamma\left(1+\alpha_{1}-\beta_{1}\right)}{\Gamma\left(\alpha_{1}+\beta_{1}\right)} \\
\zeta^{-\alpha_{1}} \psi\left(-\alpha_{1}-\beta_{1}, 1-2 \alpha_{1}, e^{-i \pi} \zeta\right) e^{-i \pi \alpha_{1}} e^{\zeta / 2}
\end{array}\right),
$$

where $\psi(a, c ; x)$ is the confluent hypergeometric function of the second kind defined above, and $\Gamma$ is the Euler's $\Gamma$-function. The solution in the other sectors is given by successive application of the jump conditions from $\left(\Psi_{1} 2\right)$ to (117).

This solution is matched with $N$ (see (82)) on the boundary $\partial U_{z_{1}}$ for large $n$. The limit $n \rightarrow$ $\infty, z \in \partial U_{z_{1}}$, corresponds to $\zeta \rightarrow \infty$. Thus, an asymptotic expansion of $\Psi_{1}$ is found using the classical result for the confluent hypergeometric function (which is (116) rewritten),

$$
\psi(a, c ; x)=x^{-a}\left[1-a(1+a-c) x^{-1}+\mathcal{O}\left(x^{-2}\right)\right],|x| \rightarrow \infty,-3 \pi / 2<\arg x<3 \pi / 2 .
$$

These asymptotics can be taken both for $\psi(a, c ; \zeta)$ and $\psi\left(a, c ; e^{-i \pi} \zeta\right)$ for $\zeta \in \mathrm{I}$ (recall the branches are fixed by the condition $0<\arg \zeta<2 \pi$. Using (118) to (117), we obtain the relevant asymptotics, which due to the triangular structure of the jump matrices remain the same in the sectors I and II,

$$
\begin{aligned}
& \Psi_{1}^{(\mathrm{I})}(\zeta)=\Psi_{1}^{(\mathrm{II})}(\zeta) \\
& =\left[I+\frac{1}{\zeta}\left(\begin{array}{cc}
\alpha_{1}^{2}-\beta_{1}^{2} & \frac{\Gamma\left(1+\alpha_{1}-\beta_{1}\right)}{\Gamma\left(\alpha_{1}+\beta_{1}\right)} e^{i \pi\left(\beta_{1}+4 \alpha_{1}\right)} \\
-\frac{\Gamma\left(1+\alpha_{1}+\beta_{1}\right)}{\Gamma\left(\alpha_{1}-\beta_{1}\right)} e^{-i \pi\left(\beta_{1}+4 \alpha_{1}\right)} & -\left(\alpha_{1}^{2}-\beta_{1}^{2}\right)
\end{array}\right)+\mathcal{O}\left(\zeta^{-2}\right)\right] \\
& \times \zeta^{-\beta_{1} \sigma_{3}} e^{-\zeta \sigma_{3} / 2}\left(\begin{array}{cc}
e^{i \pi\left(2 \beta_{1}+\alpha_{1}\right)} & 0 \\
0 & e^{-i \pi\left(\beta_{1}+2 \alpha_{1}\right)}
\end{array}\right), \\
& \zeta \rightarrow \infty, \zeta \in \mathrm{I}, \mathrm{II}, \alpha_{1} \pm \beta_{1} \neq-1,-2, \ldots .
\end{aligned}
$$

The asymptotics in the remaining sectors can be found using the relevant jump matrices.

7.6. Parametrix at point $z_{0}=1$. Before proceeding with the local parametrix near the point $z_{0}$, we first look at the Riemann-Hilbert problem for $\Psi(z)$ - the problem for Painlevé V-which will play a crucial role in finding the solution to the problem for the local parametrix. This problem was presented and solved in [3, Section 1.3], we provide a short summary of it here for the benefit of the reader. We will also use some of the details from [3, Section 4.1,4.2] to compute some additional details that are relevant to solving our problem.

The parametrix $P_{z_{0}}$ will be constructed for $0<t<t_{0}$, where $t_{0}$ is sufficiently small. Similarly to the paramtetrix at $z_{1}$, it will satisfy the same jump conditions as the R-H problem for $S(z)$ in the neighborhood $U_{z_{0}}$ of $z_{0}$, which has a sufficiently small, fixed radius. And we will have a matching condition with the R-H problem for $N(z)$ on the boundary of $U_{z_{0}}$ (referred to as $\partial U_{z_{0}}$, which will be determined by an analytic (in $U_{z_{0}}$ ) matrix function $E(z)$ ).

The Parametrix at $z_{0}$. Assume that $\Psi_{0}(\zeta)$ solves the R-H problem for $\Psi_{0}$ defined in Section 7.7 and define

$$
\Phi(\lambda ; x)=e^{\frac{x}{4} \sigma_{3}} x^{-\beta_{0} \sigma_{3}} \Psi_{0}\left(\frac{\lambda}{x}+\frac{1}{2} ; x\right) G(\lambda ; x)^{\frac{1}{2} \sigma_{3}} e^{ \pm \frac{\pi i}{2}\left(\alpha_{0}-\beta_{0}\right) \sigma_{3}}, \quad \text { for } \pm \Im \lambda>0,
$$

where

$$
G(\lambda ; x)=\left(\lambda+\frac{x}{2}\right)^{-\left(\alpha_{0}-\beta_{0}\right)}\left(\lambda-\frac{x}{2}\right)^{\alpha_{0}+\beta_{0}} e^{\lambda} e^{-\pi i\left(\alpha_{0}-\beta_{0}\right)}, \quad x>0,
$$

which is analytic in $\mathbb{C} \backslash((-\infty,-x / 2] \cup[x / 2,+\infty))$. We choose $-\pi<\arg (\lambda+x / 2)<\pi$ and $0<\arg (\lambda-x / 2)<2 \pi$. The matrix function $\Phi=\Phi(\lambda ; x)$ solves the following $\mathrm{R}-\mathrm{H}$ problem for $x>0$.

Riemann-Hilbert problem for $\Phi$ 


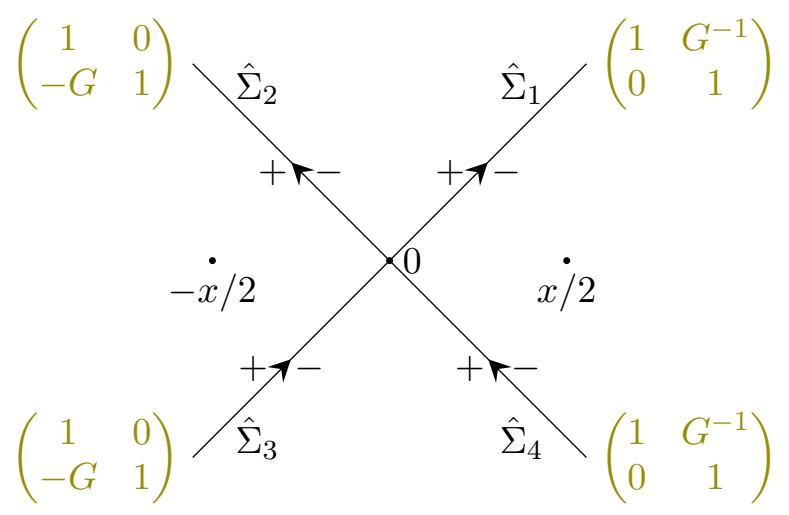

FigURE 7. Jump contour and jump matrices for the $\Phi-\mathrm{R}-\mathrm{H}$ problem

(Ф1) $\Phi: \mathbb{C} \backslash \cup_{j=1}^{4} e^{\pi i(2 j-1) / 4} \mathbb{R}^{+} \rightarrow \mathbb{C}^{2 \times 2}$ is analytic, with the rays $e^{\pi i(2 j-1) / 4} \mathbb{R}^{+}$oriented as shown in Figure 7 .

(Ф2) $\Phi$ has continuous boundary values on $\cup_{j=1}^{4} e^{\pi i(2 j-1) / 4} \mathbb{R}^{+} \backslash\{0\}$, and they are related by the following jump conditions,

(\$4) $\Phi$ is bounded near 0 .

$$
\begin{aligned}
& \Phi_{+}(\lambda)=\Phi_{-}(\lambda)\left(\begin{array}{cc}
1 & G(\lambda ; x)^{-1} \\
0 & 1
\end{array}\right), \quad \text { as } \lambda \in e^{\pi i / 4} \mathbb{R}^{+} \cup e^{7 \pi i / 4} \mathbb{R}^{+}, \\
& \Phi_{+}(\lambda)=\Phi_{-}(\lambda)\left(\begin{array}{cc}
1 & 0 \\
-G(\lambda ; x) & 1
\end{array}\right), \quad \text { as } \lambda \in e^{3 \pi i / 4} \mathbb{R}^{+} \cup e^{5 \pi i / 4} \mathbb{R}^{+}
\end{aligned}
$$

$\Phi$ has the following behavior as $\lambda \rightarrow \infty$,

$$
\Phi(\lambda)=I+\mathcal{O}\left(\lambda^{-1}\right) .
$$

Proposition 9 ([3, Proposition 3.1]).

- If $\operatorname{Re} \alpha_{0}>-1 / 2$, the $R$-H problem for $\Phi$ is uniquely solvable for all but possibly a finite number of positive $x$-values $\left\{x_{1}, \ldots, x_{k}\right\}$, where $x_{j}=x_{j}\left(\alpha_{0}, \beta_{0}\right)$ and $k=k(\alpha, \beta)$

- If $\alpha_{0}>-1 / 2$ and $\operatorname{Re} \beta_{0}=0$, the $R$-H problem for $\Phi$ is uniquely solvable for all $x>0$.

- If $\operatorname{Re} \alpha_{0}>-1 / 2$, the asymptotic condition (124) for $\Phi$ is valid uniformly for $x \in(0,+\infty)$ provided that $x$ remains bounded away from the set $\left\{x_{1}, \ldots, x_{k}\right\}$.

We now transform the jump matrices for $\Phi$ into the jump matrices for $S$ near $z_{0}=1$. First, notice that the off-diagonal entries of the jump matrices for $\Phi$ have branch points at $\pm x / 2$, and the ones for $S$ have the branch points at $e^{ \pm t}$. We thus define a conformal map $\lambda$ in the neighborhood of $z_{0}$ by

$$
\lambda(z)=\frac{x}{2 t} \log (z), \quad z \in U_{z_{0}}
$$

which maps $e^{-t}$ to $-x / 2, e^{t}$ to $x / 2$ and 1 to 0 . Again, we take the branch of the logarithm such that $\log z>0$ for $z>1$, and the branch cut is along the negative real axis. We also need that $e^{\lambda(z)}=z^{n}$ and so

$$
x=2 n t .
$$

We choose the contours $\Sigma_{1}$ and $\Sigma_{2}$ near 1 , such that $\lambda$ maps $\Sigma_{1} \cup \Sigma_{2}$ onto the jump contour $\cup_{j=1}^{4} e^{\pi i(2 j-1) / 4} \mathbb{R}^{+}$for $\Phi$. We look for the parametrix $P_{z_{0}}$ of the form

$$
P_{z_{0}}(z)=E(z) \Phi(\lambda(z) ; 2 n t) W(z),
$$


where $E$ is analytic in $U_{z_{0}}$ and $W$ is given by

$$
W(z)= \begin{cases}-G(\lambda(z))^{-\frac{1}{2} \sigma_{3}} z^{\frac{n}{2} \sigma_{3}} f_{t}(z)^{-\frac{1}{2} \sigma_{3}} \sigma_{3}, & \text { for }|z|<1 \\ G(\lambda(z))^{-\frac{1}{2} \sigma_{3}} z^{\frac{n}{2} \sigma_{3}} f_{t}(z)^{\frac{1}{2} \sigma_{3}} \sigma_{1}, & \text { for }|z|>1\end{cases}
$$

Note that $W$ is analytic in $U_{z_{0}} \backslash \mathbb{T}$, as $\lambda\left(e^{t}\right)=x / 2$ and $\lambda\left(e^{-t}\right)=-x / 2$, and the branch points of $G$ cancel out with the branch points of $f$.

If $E$ is analytic in $U_{z_{0}}$ then $P_{z_{0}}$ satisfies the same jump conditions as the matrix $S$ with the jump conditions given in the R-H problem for $S$.

The R-H problem for $\Phi$ is not solvable for a finite set of values $\left\{x_{1}, \ldots, x_{k}\right\}$ and thus we need the condition that $x=2 n t$ is distinct from each $x_{k}$; for more details, see [3, Theorem 1.1].

We now fix the matrix $E$ in a way that makes the parametrix $P_{z_{0}}$ agree with the R-H problem for $N$ on the boundary of $U_{z_{0}}$. Note that

$$
|\lambda(z)|=|n \log z|=n|\log z|>c n \quad \text { for } z \in \partial U_{z_{0}} \text {, and } c>0 .
$$

Thus, if $2 n t$ stays bounded away from $\left\{x_{1}, \ldots, x_{k}\right\}$ as $n \rightarrow \infty$, then by Proposition 9, we can use the asymptotic behavior of $\Phi$ in (124) to get

$$
P_{z_{0}}(z)=E(z)\left(I+\mathcal{O}\left(n^{-1}\right)\right) W(z) \quad \text { as } z \rightarrow \infty
$$

uniformly for $0<t<t_{0}$ and $z \in \partial U_{z_{0}}$. If $t_{0}$ is sufficiently small, we can assume that the points $e^{ \pm t}$ lie inside $U_{z_{0}}$ at a distance which is bounded from below from $\partial U_{z_{0}}$. By (121) and (128),

$$
W(z)=n^{-\beta_{0}}\left\{\begin{array}{cc}
\left(\begin{array}{cc}
\mathcal{O}(1) & 0 \\
0 & \mathcal{O}(1)
\end{array}\right), & |z|<1, \\
0 & \mathcal{O}(1) \\
\mathcal{O}(1) & 0
\end{array}\right), \quad|z|>1,
$$

as $n \rightarrow \infty$ uniformly for $0<t<t_{0}$ and uniformly for $z \in \partial U_{z_{0}} \backslash \mathbb{T}$. We set

$$
E(z)=N(z) W(z)^{-1},
$$

it can be verified using the jumps for $N$ and $W$ across $\mathbb{T}$ that $E$ is analytic in the whole neighborhood $\bar{U}_{z_{0}}$ of 1 . Using (80) and (130), we have

$$
E(z)=\left(\begin{array}{cc}
0 & \mathcal{O}(1) \\
\mathcal{O}(1) & 0
\end{array}\right) n^{\beta_{0} \sigma_{3}}
$$

as $n \rightarrow \infty$ uniformly for $0<t<t_{0}$ and $z \in \partial U_{z_{0}}$. Therefore, using (129), we obtain the following asymptotics for the matching condition for $z \in \partial U_{z_{0}}$ :

$$
P_{z_{0}}(z) N(z)^{-1}=E(z)\left(I+\mathcal{O}\left(n^{-1}\right)\right) E(z)^{-1}=I+n^{-\beta_{0} \sigma_{3}} \mathcal{O}\left(n^{-1}\right) n^{\beta_{0} \sigma_{3}} \quad \text { as } n \rightarrow \infty
$$

uniformly for $0<t<t_{0}$ and if $2 n t$ is away from the set $\left\{x_{1}, \ldots, x_{k}\right\}$.

We also note here that $S P_{z_{0}}^{-1}$ is analytic in the neighborhood of $U_{z_{0}}$, which we need for the final R-H problem in Section 7.8. Since $P_{z_{0}}$ has the same jumps as $S$ inside $U_{z_{0}}$, it follows that any singularity at $z=1$ is removable.

Similarly to the parametrix at $z_{1}$, we will now compute the first correction term $\Delta_{1}$ in the asymptotic series in inverse powers of $n$

$$
P_{z_{0}}(z) N^{-1}(z)=I+\Delta_{1}(z)+n^{-\operatorname{Re} \beta_{0} \sigma_{3}} \mathcal{O}\left(n^{-2}\right) n^{\operatorname{Re} \beta_{0} \sigma_{3}} .
$$


Using (80), (127), and (131), we get (135)

$$
P_{z_{0}}(z) N^{-1}(z)= \begin{cases}D(z)^{\sigma_{3}}\left(\begin{array}{cc}
0 & 1 \\
-1 & 0
\end{array}\right) W(z)^{-1} \Phi(\lambda(z) ; x) W(z)\left(\begin{array}{cc}
0 & -1 \\
1 & 0
\end{array}\right) D(z)^{-\sigma_{3}}, & \text { for }|z|<1, \\
D(z)^{\sigma_{3}} W(z)^{-1} \Phi(\lambda(z) ; x) W(z) D(z)^{-\sigma_{3},} & \text { for }|z|>1 .\end{cases}
$$

Denote the elements of $\Phi(\lambda(z) ; x)$ by $\Phi_{i j}(z)$ for $i, j=1,2$ and write $G(z)$ for $G(\lambda(z)$. By (128), (136)

$$
P_{z_{0}}(z) N^{-1}(z)=\left\{\begin{array}{cc}
\left(\begin{array}{cc}
\Phi_{22}(z) & D(z)^{2} G^{-1}(z) z^{n} f_{t}(z)^{-1} \Phi_{21}(z) \\
D(z)^{-2} G(z) z^{-n} f_{t}(z) \Phi_{12}(z) & \Phi_{11}(z) \\
\Phi_{22}(z) & D(z)^{2} G^{-1}(z) z^{n} f_{t}(z) \Phi_{21}(z) \\
D(z)^{-2} G(z) z^{-n} f_{t}(z)^{-1} \Phi_{12}(z) & \text { for }|z|<1
\end{array}\right) & \text { for }|z|>1 \\
\Phi_{11}(z)
\end{array}\right.
$$

We now look at the behavior of these as $z \rightarrow e^{\mp t}$ in the two cases $|z|<1$ and $|z|>1$. We denote by $u=z-e^{\mp t}$ and take $u \rightarrow 0$. First recall the Szegö function (79) and (81), and observe

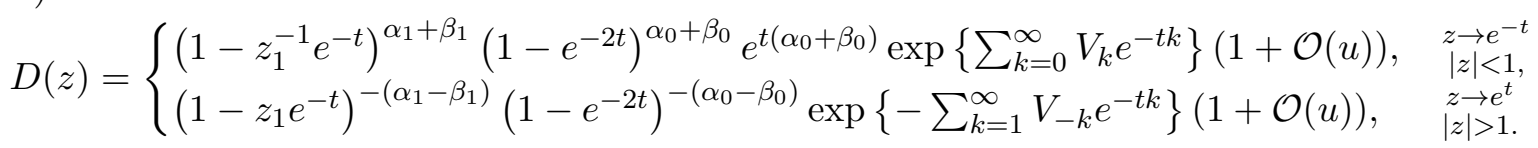

By 121,

$$
\begin{aligned}
G(\lambda(z) ; x=2 n t)=n^{2 \beta_{0}}\left(\log \frac{z}{e^{-t}}\right)^{-\left(\alpha_{0}-\beta_{0}\right)}\left(\log \frac{z}{e^{t}}\right)^{\alpha_{0}+\beta_{0}} z^{n} e^{-i \pi\left(\alpha_{0}-\beta_{0}\right)}, \\
\quad= \begin{cases}n^{2 \beta_{0}} u^{-\left(\alpha_{0}-\beta_{0}\right)}(2 t)^{\alpha_{0}+\beta_{0}} e^{-t n} e^{-t\left(\alpha_{0}-\beta_{0}\right)} e^{-2 \pi i \beta_{0}}(1+\mathcal{O}(u)), & u \rightarrow 0 \\
n^{2 \beta_{0}} u^{\alpha_{0}+\beta_{0}}(2 t)^{-\left(\alpha_{0}-\beta_{0}\right)} e^{t n} e^{-t\left(\alpha_{0}+\beta_{0}\right)} e^{-i \pi\left(\alpha_{0}-\beta_{0}\right)}(1+\mathcal{O}(u)), & u \rightarrow 0 \\
z \rightarrow e^{t}\end{cases}
\end{aligned}
$$

Now considering the symbol (38) and (84),

$$
f(z ; t)=\left\{\begin{array}{cc}
e^{V\left(e^{-t}\right)}\left(1-z_{1}^{-1} e^{-t}\right)^{2 \alpha_{1}}\left(1-e^{-2 t}\right)^{\alpha_{0}+\beta_{0}} u^{\alpha_{0}-\beta_{0}} e^{t\left(\alpha_{1}-\beta_{1}\right)} & \\
\times e^{2 t \alpha_{0}} e^{i \pi\left(\alpha_{1}-\beta_{1}\right)} z_{1}^{\alpha_{1}-\beta_{1}}(1+\mathcal{O}(u)), & \substack{u \rightarrow 0 \\
z \rightarrow e^{-t},} \\
e^{V\left(e^{t}\right)}\left(1-z_{1} e^{-t}\right)^{2 \alpha_{1}}\left(1-e^{-2 t}\right)^{\alpha_{0}+\beta_{0}} u^{\alpha_{0}+\beta_{0}} e^{t\left(\alpha_{1}+\beta_{1}\right)} & \\
\times e^{-i \pi\left(\alpha_{1}+\beta_{1}\right)} e^{-i \pi\left(\alpha_{1}+\beta_{1}\right)} z_{1}^{-\left(\alpha_{1}+\beta_{1}\right)}(1+\mathcal{O}(u)), & \substack{u \rightarrow 0 \\
z \rightarrow e^{t}}
\end{array}\right.
$$

In what follows, we only need one of the entries of $\Delta_{1}(z)$; the other elements can be easily computed similarly using the information above. Combining the above with (127), we get

$$
\begin{aligned}
\left(\Delta_{1}(z)\right)_{12}= & \left(1-z_{1}^{-1} e^{-t}\right)^{2 \beta_{1}}\left(1-e^{-2 t}\right)^{\alpha_{0}+\beta_{0}} \exp \left\{\sum_{k=0}^{\infty} V_{k} e^{-t k}\right\} \exp \left\{-\sum_{k=1}^{\infty} V_{-k} e^{t k}\right\} \\
& \times e^{t\left(\alpha_{0}+\beta_{0}\right)} e^{-t\left(\alpha_{1}-\beta_{1}\right)} e^{-2 \pi i \beta_{0}} e^{-i \pi\left(\alpha_{1}-\beta_{1}\right)} z_{1}^{-\left(\alpha_{1}-\beta_{1}\right)}(2 t)^{-\left(\alpha_{0}+\beta_{0}\right)} n^{-2 \beta_{0}} \\
& \times \Phi_{21}\left(\lambda\left(z \rightarrow e^{-t}\right) ; x=2 n t\right)(1+\mathcal{O}(u)) \text { as } z \rightarrow e^{-t}
\end{aligned}
$$

and

$$
\begin{aligned}
\left(\Delta_{1}(z)\right)_{12}= & \left(1-z_{1} e^{-t}\right)^{2 \beta_{1}}\left(1-e^{-2 t}\right)^{-\left(\alpha_{0}-\beta_{0}\right)} \exp \left\{-\sum_{k=1}^{\infty} V_{-k} e^{-t k}\right\} \exp \left\{\sum_{k=0}^{\infty} V_{k} e^{t k}\right\} \\
& \times e^{t\left(\alpha_{0}+\beta_{0}\right)} e^{t\left(\alpha_{1}+\beta_{1}\right)} e^{-2 \pi i \beta_{0}} e^{-i \pi\left(\alpha_{1}+\beta_{1}\right)} z_{1}^{-\left(\alpha_{1}+\beta_{1}\right)}(2 t)^{\alpha_{0}-\beta_{0}} n^{-2 \beta_{0}} \\
& \times \Phi_{21}\left(\lambda\left(z \rightarrow e^{t}\right) ; x=2 n t\right)(1+\mathcal{O}(u)) \text { as } z \rightarrow e^{t}
\end{aligned}
$$


It remains to find the appropriate asymptotics for $\Phi(\lambda, x)$. We will find two asymptotic expansions of the form

$$
\Phi\left(\lambda^{\prime}\right)=I+\frac{C_{1}}{\lambda^{\prime}}+\mathcal{O}\left(\left(\lambda^{\prime}\right)^{-2}\right) \quad \text { as } \lambda^{\prime} \rightarrow \infty .
$$

One of these will hold uniformly for $0<x<\delta$ and the other will hold uniformly for $x>C$, where $\delta, C>0$ and $\lambda^{\prime}(z)=\lambda(z) \pm \frac{x}{2}$. After that, judging by the similarities in the two asymptotic expansions, we will attempt to find a constant $C_{1}$ that will asymptotically match both cases.

Asymptotics for $\Phi$ as $x \rightarrow \infty$. We use the following expansion given in Section 4.1 of [3]:

$$
\tilde{\Phi}(\zeta)=\Phi(x \zeta=\lambda ; x)=I+\frac{\tilde{C}_{1}}{\zeta}+\mathcal{O}\left(\zeta^{-2}\right) \text { as } \zeta \rightarrow \infty
$$

where

$$
\tilde{C}_{1}=\left(\begin{array}{cc}
\frac{x^{-2+2 \alpha} e^{-x}}{\Gamma\left(\alpha_{0}-\beta_{0}\right) \Gamma\left(\alpha_{0}+\beta_{0}\right)}\left(1+\mathcal{O}\left(\frac{1}{x}\right)\right) & -x^{-1+\alpha_{0}-\beta_{0}} e^{-x / 2} \frac{e^{-2 \pi i \beta_{0}}}{\Gamma\left(\alpha_{0}+\beta_{0}\right)}\left(1+\mathcal{O}\left(\frac{1}{x}\right)\right) \\
x^{-1+\alpha_{0}+\beta_{0}} e^{-x / 2} \frac{e^{2 \pi i \beta_{0}}}{\Gamma\left(\alpha_{0}-\beta_{0}\right)}\left(1+\mathcal{O}\left(\frac{1}{x}\right)\right) & \frac{-x^{-2+2 \alpha} e^{-x}}{\Gamma\left(\alpha_{0}-\beta_{0}\right) \Gamma\left(\alpha_{0}+\beta_{0}\right)}\left(1+\mathcal{O}\left(\frac{1}{x}\right)\right)
\end{array}\right) .
$$

Via the transformation $\zeta \mapsto \frac{\lambda}{x}$, we get

$$
\Phi_{21}(\lambda ; x)=x^{\alpha_{0}+\beta_{0}} e^{-x / 2} \frac{e^{2 \pi i \beta_{0}}}{\Gamma\left(\alpha_{0}-\beta_{0}\right)} \lambda^{-1}\left(1+\mathcal{O}\left(\frac{1}{x}\right)\right)+\mathcal{O}\left(\frac{x^{2}}{\lambda^{2}}\right) \text { as } \lambda \rightarrow \infty
$$

uniformly for $x>C>0$. Translating $\lambda$ by $\pm \frac{x}{2}$ makes no difference, and so

$$
\Phi_{21}\left(\lambda^{\prime} ; x\right)=x^{\alpha_{0}+\beta_{0}} e^{-x / 2} \frac{e^{2 \pi i \beta_{0}}}{\Gamma\left(\alpha_{0}-\beta_{0}\right)}\left(\lambda^{\prime}\right)^{-1}\left(1+\mathcal{O}\left(\frac{1}{x}\right)\right)+\mathcal{O}\left(\left(\frac{x}{\lambda^{\prime}}\right)^{2}\right) \text { as } \lambda^{\prime} \rightarrow \infty
$$

uniformly for $x>C>0$.

Asymptotics for $\Phi$ as $x \rightarrow 0$. We use the approach in Section 4.2 of [3] to find asymptotics of $\Psi_{0}$ and $\Phi$ as $x \rightarrow 0$. Recall the R-H problem for $\Psi_{0}$ in (165)-(173), which is intimately connected to the function $\Phi(\lambda(z) ; x)$ via 120 .

We now use $(199)$ to find asymptotics of $\widehat{\Psi}(\widehat{\lambda})$ (a transformation of the R-H problem for $\Psi_{0}$ defined in Section 7.7) as $\widehat{\lambda}:=\lambda-\frac{x}{2} \rightarrow \infty$. We rewrite (120) and (121) using $\lambda=\widehat{\lambda}+\frac{x}{2}$ (see the discussion below (178)). We obtain,

$$
\Phi\left(\lambda=\widehat{\lambda}+\frac{x}{2} ; x\right)=e^{x / 4 \sigma_{3}} x^{-\beta_{0} \sigma_{3}} \Psi\left(\frac{\hat{\lambda}}{x}+1 ; x\right) G\left(\widehat{\lambda}+\frac{x}{2}, x\right)^{\frac{1}{2} \sigma_{3}} e^{\frac{i \pi}{2}\left(\alpha_{0}-\beta_{0}\right) \sigma_{3}},
$$

and

$$
G\left(\widehat{\lambda}+\frac{x}{2} ; x\right)=(\widehat{\lambda}+x)^{-\left(\alpha_{0}-\beta_{0}\right)} \widehat{\lambda}^{\alpha_{0}+\beta_{0}} e^{\widehat{\lambda}} e^{x / 2} e^{-i \pi\left(\alpha_{0}-\beta_{0}\right)} .
$$

Now using (178) and (199),

$$
\begin{aligned}
\Phi(\widehat{\lambda} ; x) & =e^{-x / 4 \sigma_{3}} \widehat{\Psi}(\widehat{\lambda} ; x) G\left(\widehat{\lambda}+\frac{x}{2} ; x\right)^{\frac{1}{2} \sigma_{3}} e^{\frac{i \pi}{2}\left(\alpha_{0}-\beta_{0}\right) \sigma_{3}} \\
& =e^{-x / 4 \sigma_{3}} \widehat{R}(\widehat{\lambda}) M(\widehat{\lambda}) G\left(\widehat{\lambda}+\frac{x}{2} ; x\right)^{\frac{1}{2} \sigma_{3}} e^{\frac{i \pi}{2}\left(\alpha_{0}-\beta_{0}\right) \sigma_{3}} \quad \text { for } \widehat{\lambda} \in \mathbb{C} \backslash U_{\varepsilon} .
\end{aligned}
$$

Using the asymptotic behavior of $\widehat{R}(\widehat{\lambda})=I+\mathcal{O}\left(\widehat{\lambda}^{-1}\right)$ and writing $G=G\left(\widehat{\lambda}+\frac{x}{2}\right)$, we obtain

$$
\Phi(\widehat{\lambda} ; x)=\left(\begin{array}{cc}
e^{-x / 4}\left(M_{11}\left(1+\mathcal{O}\left(\widehat{\lambda}^{-1}\right)\right)+M_{21} \mathcal{O}\left(\widehat{\lambda}^{-1}\right)\right) & e^{-x / 4}\left(M_{12}\left(1+\mathcal{O}\left(\widehat{\lambda}^{-1}\right)\right)+M_{22} \mathcal{O}\left(\widehat{\lambda}^{-1}\right)\right) \\
e^{x / 4}\left(M_{11}\left(1+\mathcal{O}\left(\widehat{\lambda}^{-1}\right)\right)+M_{21} \mathcal{O}\left(\widehat{\lambda}^{-1}\right)\right) & e^{x / 4}\left(M_{12}\left(1+\mathcal{O}\left(\widehat{\lambda}^{-1}\right)\right)+M_{22} \mathcal{O}\left(\widehat{\lambda}^{-1}\right)\right)
\end{array}\right)
$$




$$
=\left(\begin{array}{cc}
e^{-x / 4} G^{\frac{1}{2}} e^{\frac{i \pi}{2}\left(\alpha_{0}-\beta_{0}\right)} M_{11}\left(\left(1+\frac{M_{21}}{M_{11}} \mathcal{O}\left(\widehat{\lambda}^{-1}\right)\right)\right. & e^{-x / 4} G^{-\frac{1}{2}} e^{-\frac{i \pi}{2}\left(\alpha_{0}-\beta_{0}\right)} M_{12}\left(\left(1+\frac{M_{22}}{M_{12}} \mathcal{O}\left(\widehat{\lambda}^{-1}\right)\right)\right. \\
e^{x / 4} G^{\frac{1}{2}} e^{\frac{i \pi}{2}\left(\alpha_{0}-\beta_{0}\right)} M_{21}\left(\left(1+\frac{M_{11}}{M_{21}} \mathcal{O}\left(\widehat{\lambda}^{-1}\right)\right)\right. & e^{x / 4} G^{-\frac{1}{2}} e^{-\frac{i \pi}{2}\left(\alpha_{0}-\beta_{0}\right)} M_{22}\left(\left(1+\frac{M_{12}}{M_{22}} \mathcal{O}\left(\widehat{\lambda}^{-1}\right)\right)\right.
\end{array}\right) .
$$

We can find the asymptotics of each $M_{i j}$ as $\widehat{\lambda} \rightarrow \infty$, but we only concentrate on finding the details of $M_{21}$ (finding details of every $M_{i j}$ shows that the ratios above are $\mathcal{O}(1)$ ). By (194) and (195),

$$
M_{21}=e^{i \pi\left(\beta_{0}+3 \alpha_{0}\right)} e^{-\widehat{\lambda} / 2 \widehat{\lambda}^{-\alpha_{0}}} \psi\left(1-\alpha_{0}+\beta_{0}, 1-2 \alpha_{0}, \widehat{\lambda}\right) e^{i \pi\left(\beta_{0}-3 \alpha_{0}\right)} \frac{\Gamma\left(1+\alpha_{0}+\beta_{0}\right)}{\Gamma\left(\alpha_{0}-\beta_{0}\right)} .
$$

Using the asymptotics for the confluent hypergeometric function $\psi$ from (118), we obtain

$$
M_{21}=e^{2 i \pi \beta_{0}} e^{-\widehat{\lambda} / 2} \frac{\Gamma\left(1+\alpha_{0}+\beta_{0}\right)}{\Gamma\left(\alpha_{0}-\beta_{0}\right)} \widehat{\lambda}^{-\beta_{0}} \widehat{\lambda}^{-1}\left(1+\mathcal{O}\left(\widehat{\lambda}^{-1}\right)\right) .
$$

Thus, by 148, 149, and 151,

$$
\begin{aligned}
\Phi_{21}(\widehat{\lambda}) & =e^{x / 4} e^{2 i \pi \beta_{0}}(\widehat{\lambda}+x)^{\left(\frac{\alpha_{0}-\beta_{0}}{2}\right)} \widehat{\lambda}^{\left(\frac{\alpha_{0}+\beta_{0}}{2}\right)} e^{\widehat{\lambda} / 2} e^{x / 4} e^{-\widehat{\lambda} / 2} \frac{\Gamma\left(1+\alpha_{0}+\beta_{0}\right)}{\Gamma\left(\alpha_{0}-\beta_{0}\right)} \widehat{\lambda}^{-\beta_{0}} \widehat{\lambda}^{-1}\left(1+\mathcal{O}\left(\widehat{\lambda}^{-1}\right)\right) \\
& =e^{x / 2} e^{2 i \pi \beta_{0}} \frac{\Gamma\left(1+\alpha_{0}+\beta_{0}\right)}{\Gamma\left(\alpha_{0}-\beta_{0}\right)}\left(\widehat{\lambda}^{-\left(\frac{\alpha_{0}-\beta_{0}}{2}\right)}\left(1+\frac{x}{\widehat{\lambda}}\right)^{-\left(\frac{\alpha_{0}-\beta_{0}}{2}\right)} \widehat{\lambda}^{\frac{\alpha_{0}-\beta_{0}}{2}}\right) \widehat{\lambda}^{-1}\left(1+\mathcal{O}\left(\widehat{\lambda}^{-1}\right)\right) \\
152) \quad & =e^{x / 2} e^{2 i \pi \beta_{0}} \frac{\Gamma\left(1+\alpha_{0}+\beta_{0}\right)}{\Gamma\left(\alpha_{0}-\beta_{0}\right)} \widehat{\lambda}^{-1}\left(1+\mathcal{O}\left(\widehat{\lambda}^{-1}\right)\right) \quad \text { as } \widehat{\lambda} \rightarrow \infty,
\end{aligned}
$$

uniformly for $0<x<\delta, \delta>0$. A simple translation $\widehat{\lambda} \mapsto \tilde{\lambda}-x$ shows that

$$
\begin{aligned}
\Phi_{21}(\tilde{\lambda})= & e^{x / 4} G^{1 / 2}(\tilde{\lambda}-x ; x) e^{i \pi / 2\left(\alpha_{0}-\beta_{0}\right)} M_{21}(\tilde{\lambda}-x)\left(1+\mathcal{O}\left(\tilde{\lambda}^{-1}\right)\right) \\
= & \left.e^{x / 4}\left(\tilde{\lambda}^{-\left(\frac{\alpha_{0}-\beta_{0}}{2}\right)}(\tilde{\lambda}-x)\left(\frac{\alpha_{0}+\beta_{0}}{2}\right) e^{\tilde{\lambda} / 2} e^{-x / 4} e^{-i \pi / 2\left(\alpha_{0}-\beta_{0}\right.}\right)\right) e^{i \pi / 2\left(\alpha_{0}-\beta_{0}\right)} e^{2 i \pi \beta_{0}} e^{-\tilde{\lambda} / 2} \\
& \times \frac{\Gamma\left(1+\alpha_{0}+\beta_{0}\right)}{\Gamma\left(\alpha_{0}-\beta_{0}\right)}(\tilde{\lambda}-x)^{-1-\beta_{0}}\left(1+\mathcal{O}\left(\tilde{\lambda}^{-1}\right)\right) \\
= & e^{x / 2} e^{2 i \pi \beta_{0}} \frac{\Gamma\left(1+\alpha_{0}+\beta_{0}\right)}{\Gamma\left(\alpha_{0}-\beta_{0}\right)}\left(\tilde{\lambda}^{-\left(\frac{\alpha_{0}-\beta_{0}}{2}\right)}\left(\tilde{\lambda}\left(1-\frac{x}{\tilde{\lambda}}\right)\right)^{\left(\frac{\alpha_{0}-\beta_{0}}{2}\right)} \tilde{\lambda}^{-1}\left(1-\frac{x}{\tilde{\lambda}}\right)^{-1}\right)\left(1+\mathcal{O}\left(\tilde{\lambda}^{-1}\right)\right)
\end{aligned}
$$

$$
=e^{x / 2} e^{2 i \pi \beta_{0}} \frac{\Gamma\left(1+\alpha_{0}+\beta_{0}\right)}{\Gamma\left(\alpha_{0}-\beta_{0}\right)} \tilde{\lambda}^{-1}\left(1+\mathcal{O}\left(\tilde{\lambda}^{-1}\right)\right) \quad \text { as } \tilde{\lambda} \rightarrow \infty
$$

uniformly for $0<x<\delta, \delta>0$.

Expression for a fixed $x$. The function

$$
K(x)=e^{x / 2} \int_{x}^{\infty} y^{\alpha_{0}+\beta_{0}} e^{-y} d y
$$

has the following behavior:

$$
K(x) \sim \begin{cases}e^{-x / 2} x^{\alpha_{0}+\beta_{0}}, & \text { as } x \rightarrow \infty \\ e^{x / 2} \Gamma\left(\alpha_{0}+\beta_{0}+1\right), & \text { as } x \rightarrow 0\end{cases}
$$


We can use $K$ to write down an expression for $\Phi_{21}\left(\lambda^{\prime} ; x\right)$ with fixed $x$, that is asymptotically valid for small and large values of $x$. From (146), 152, (153) we obtain,

$$
\Phi_{21}\left(\lambda^{\prime} ; x\right)=\frac{e^{2 \pi i \beta_{0}}}{\Gamma\left(\alpha_{0}-\beta_{0}\right)} K(x)\left(\lambda^{\prime}\right)^{-1}+\mathcal{O}\left(\left(\lambda^{\prime}\right)^{-2}\right) \quad \text { as } \lambda^{\prime} \rightarrow \infty .
$$

We also note that if $x=2 n t, \widehat{\lambda}=n \log \frac{z}{e^{t}}$ and $\tilde{\lambda}=n \log \frac{z}{e^{-t}}$ and for $t$ fixed,

$$
\frac{1}{\widehat{\lambda}}=\frac{e^{t}}{n\left(z-e^{t}\right)}+\frac{1}{2 n}+\mathcal{O}\left(z-e^{t}\right), \quad z \rightarrow e^{t},
$$

and similarly,

$$
\frac{1}{\tilde{\lambda}}=\frac{e^{-t}}{n\left(z-e^{-t}\right)}+\frac{1}{2 n}+\mathcal{O}\left(z-e^{-t}\right), \quad z \rightarrow e^{-t} .
$$

Combining the results from $(140),(141), 1152)$, and $(153)$, we obtain the final expression for $\left(\Delta_{1}(z)\right)_{12}$ in the expansion of $\overline{P_{z_{0}}}(z) N^{-1}(z)$ in inverse powers of $n$, for $z \rightarrow e^{-t}$,

$$
\begin{aligned}
& \left(\Delta_{1}(z)\right)_{12}=\widehat{\lambda}^{-1}\left(1-z_{1}^{-1} e^{-t}\right)^{2 \beta_{1}}\left(1-e^{-2 t}\right)^{\alpha_{0}+\beta_{0}} \exp \left\{\sum_{k=0}^{\infty} V_{k} e^{-t k}\right\} \exp \left\{-\sum_{k=1}^{\infty} V_{-k} e^{t k}\right\} \\
& \quad \times e^{t\left(\alpha_{0}+\beta_{0}\right)} e^{-t\left(\alpha_{1}-\beta_{1}\right)} e^{-i \pi\left(\alpha_{1}-\beta_{1}\right)} z_{1}^{-\left(\alpha_{1}-\beta_{1}\right)}(2 t)^{-\left(\alpha_{0}+\beta_{0}\right)} \frac{n^{-2 \beta_{0}}}{\Gamma\left(\alpha_{0}-\beta_{0}\right)} K(2 n t),
\end{aligned}
$$

and for $z \rightarrow e^{t}$,

$$
\begin{aligned}
& \left(\Delta_{1}(z)\right)_{12}=\tilde{\lambda}^{-1}\left(1-z_{1} e^{-t}\right)^{2 \beta_{1}}\left(1-e^{-2 t}\right)^{-\left(\alpha_{0}-\beta_{0}\right)} \exp \left\{-\sum_{k=1}^{\infty} V_{-k} e^{-t k}\right\} \exp \left\{\sum_{k=0}^{\infty} V_{k} e^{t k}\right\} \\
& \times e^{t\left(\alpha_{0}+\beta_{0}\right)} e^{t\left(\alpha_{1}+\beta_{1}\right)} e^{-i \pi\left(\alpha_{1}+\beta_{1}\right)} z_{1}^{-\left(\alpha_{1}+\beta_{1}\right)}(2 t)^{\alpha_{0}-\beta_{0}} \frac{n^{-2 \beta_{0}}}{\Gamma\left(\alpha_{0}-\beta_{0}\right)} K(2 n t) .
\end{aligned}
$$

7.7. Riemann-Hilbert Problem for Painlevé V. We consider the second order ODE

$$
\left(x \frac{d^{2} \sigma}{d x^{2}}\right)^{2}=\left(\sigma-x \frac{d \sigma}{d x}+2\left(\frac{d \sigma}{d x}\right)^{2}+2 \alpha_{0} \frac{d \sigma}{d x}\right)^{2}-4\left(\frac{d \sigma}{d x}\right)^{2}\left(\frac{d \sigma}{d x}+\alpha_{0}+\beta_{0}\right)\left(\frac{d \sigma}{d x}+\alpha_{0}-\beta_{0}\right),
$$

which is the $\sigma$-form of the Painlevé $\mathrm{V}$ equation,

$$
u_{x x}=\left(\frac{1}{2 u}+\frac{1}{u-1}\right) u_{x}^{2}-\frac{1}{x} u_{x}+\frac{(u-1)^{2}}{x^{2}}\left(A u+\frac{b}{u}\right)+\frac{C u}{x}+D \frac{u(u+1)}{u-1},
$$

which was produced by Jimbo, Miwa, Okamoto (see [17, 18]). For our R-H problem, we have

$$
A=\frac{1}{2}\left(\alpha_{0}-\beta_{0}\right)^{2}, \quad B=-\frac{1}{2}\left(\alpha_{0}+\beta_{0}\right)^{2}, \quad C=1+2 \beta_{0}, \quad D=-\frac{1}{2} .
$$

The function $\sigma(x)$ in the solution of 161 can be constructed explicitly in terms of the R-H problem below. We consider $\Gamma=\cup_{j=1}^{6} \Gamma_{j} \subset \mathbb{C}$ as the contour for this problem (see Figure 8), where

$$
\begin{array}{ccc}
\Gamma_{1}=\frac{1}{2}+e^{i \pi / 4} \mathbb{R}^{+}, & \Gamma_{2}=\frac{1}{2}+e^{i 3 \pi / 4} \mathbb{R}^{+}, & \Gamma_{3}=\frac{1}{2}+e^{i 5 \pi / 4} \mathbb{R}^{+}, \\
\Gamma_{4}=\frac{1}{2}+e^{i 7 \pi / 4} \mathbb{R}^{+}, & \Gamma_{5}=(1,+\infty), & \Gamma_{6}=(0,1) .
\end{array}
$$

The contours $\Gamma_{1}, \ldots, \Gamma_{5}$ are oriented towards infinity and $\Gamma_{6}$ is oriented to the right. As before, we assume that $\operatorname{Re} \alpha_{0}>-1 / 2$.

Riemann-Hilbert problem for $\Psi_{0}$ 


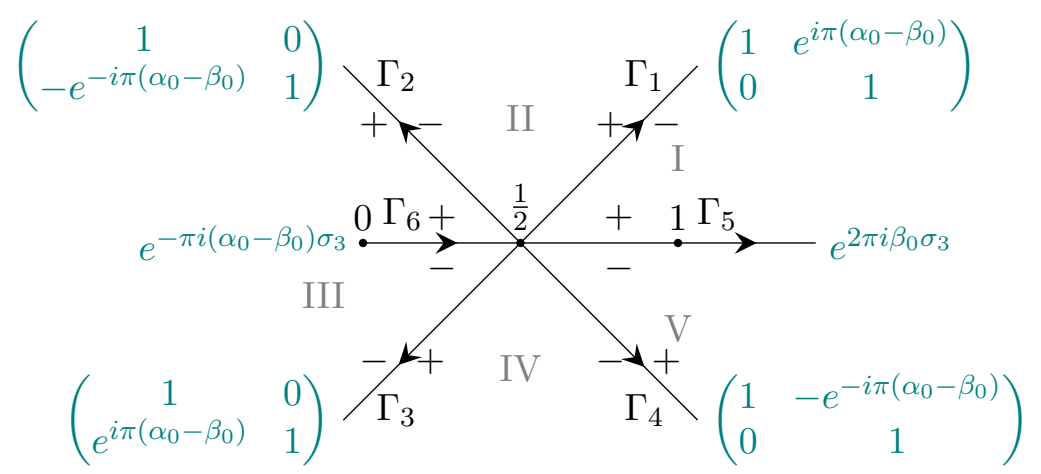

FiguRE 8. Jump contour and jump matrices for the Painleve V R-H problem for $\Psi_{0}$

$\left(\Psi_{0} 1\right) \quad \Psi_{0}: \mathbb{C} \backslash \Gamma \rightarrow \mathbb{C}^{2 \times 2}$ is analytic.

$\left(\Psi_{0} 2\right) \quad \Psi_{0}$ has continuous boundary values on $\Gamma \backslash\{0,1 / 2,1\}$, and they are related by the following jump conditions,

$$
\begin{gathered}
\Psi_{0,+}(\zeta)=\Psi_{0,-}(\zeta)\left(\begin{array}{cc}
1 & e^{\pi i\left(\alpha_{0}-\beta_{0}\right)} \\
0 & 1
\end{array}\right), \quad \text { for } \zeta \in \Gamma_{1}, \\
\Psi_{0,+}(\zeta)=\Psi_{0,-}(\zeta)\left(\begin{array}{cc}
1 & 0 \\
-e^{-\pi i\left(\alpha_{0}-\beta_{0}\right)} & 1
\end{array}\right), \quad \text { for } \zeta \in \Gamma_{2}, \\
\Psi_{0,+}(\zeta)=\Psi_{0,-}(\zeta)\left(\begin{array}{cc}
1 & 0 \\
e^{\pi i\left(\alpha_{0}-\beta_{0}\right)} & 1
\end{array}\right), \quad \text { for } \zeta \in \Gamma_{3}, \\
\Psi_{0,+}(\zeta)=\Psi_{0,-}(\zeta)\left(\begin{array}{cc}
1 & -e^{-\pi i\left(\alpha_{0}-\beta_{0}\right)} \\
0 & 1
\end{array}\right), \quad \text { for } \zeta \in \Gamma_{4}, \\
\Psi_{0,+}(\zeta)=\Psi_{0,-}(\zeta) e^{2 \pi i \beta_{0} \sigma_{3}}, \\
\Psi_{0,+}(\zeta)=\Psi_{0,-}(\zeta) e^{-\pi i\left(\alpha_{0}-\beta_{0}\right) \sigma_{3}},
\end{gathered}
$$

$\left(\Psi_{0} 3\right) \quad \Psi_{0}$ has the following asymptotic behavior as $\zeta \rightarrow \infty$, for some matrices $C_{1}=C_{1}\left(x, \alpha_{0}, \beta_{0}\right)$, $C_{2}=C_{2}\left(x, \alpha_{0}, \beta_{0}\right)$,

$$
\Psi_{0}(\zeta)=\left(I+\frac{C_{1}}{\zeta}+\frac{C_{2}}{\zeta^{2}}+\mathcal{O}\left(\zeta^{-3}\right)\right) \zeta^{-\beta_{0} \sigma_{3}} e^{-(x / 2) \zeta \sigma_{3}} .
$$

$\left(\Psi_{0} 4\right) \quad \Psi_{0}$ has the following asymptotic behavior close to the following points,

$$
\begin{array}{ll}
\left(\Psi_{0} 4.1\right) & \Psi_{0}(\zeta)=\mathcal{O}\left(\begin{array}{ll}
|\zeta|^{\left(\alpha_{0}-\beta_{0}\right) / 2} & |\zeta|^{-\left(\alpha_{0}-\beta_{0}\right) / 2} \\
|\zeta|^{\left(\alpha_{0}-\beta_{0}\right) / 2} & |\zeta|^{-\left(\alpha_{0}-\beta_{0}\right) / 2}
\end{array}\right), \quad \text { as } \zeta \rightarrow 0, \\
\left(\Psi_{0} 4.2\right) & \Psi_{0}(\zeta)=\mathcal{O}\left(\begin{array}{ll}
|\zeta-1|^{-\left(\alpha_{0}+\beta_{0}\right) / 2} & |\zeta-1|^{\left(\alpha_{0}+\beta_{0}\right) / 2} \\
|\zeta-1|^{-\left(\alpha_{0}+\beta_{0}\right) / 2} & |\zeta-1|^{\left(\alpha_{0}+\beta_{0}\right) / 2}
\end{array}\right), \quad \text { as } \zeta \rightarrow 1,
\end{array}
$$

and $\Psi_{0}$ is bounded near $\zeta=1 / 2$.

Let $L_{j}$ denote the jump matrices in the jump conditions above, for $j=1, \ldots, 6$, corresponding to each $\Gamma_{j}$. In all cases we have that $\operatorname{det} L_{j}=1$, and so, for $j=1, \ldots, 6$,

$$
\left(\operatorname{det} \Psi_{0}\right)_{+}(\zeta)=\left(\operatorname{det} \Psi_{0}\right)_{-}(\zeta) \operatorname{det} L_{j}=\left(\operatorname{det} \Psi_{0}\right)_{-}(\zeta)
$$




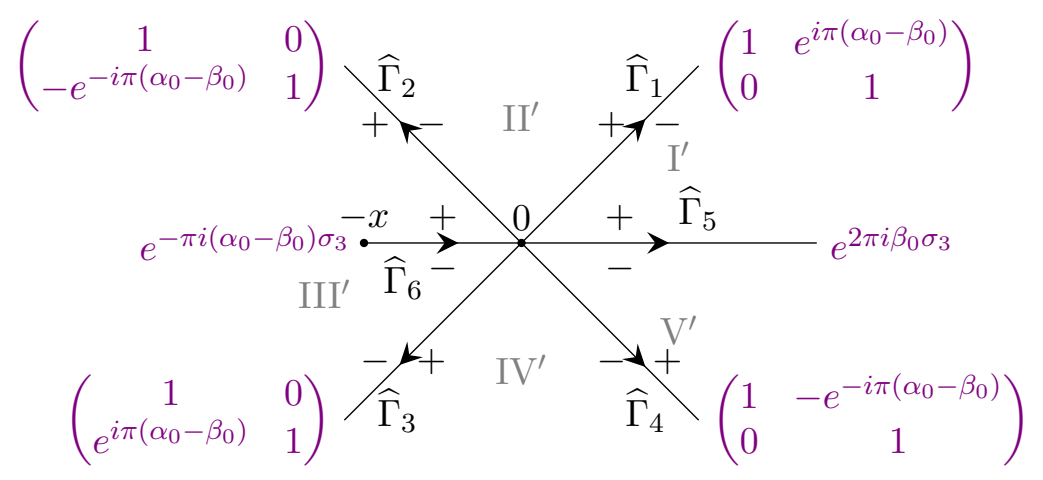

FigURE 9. Jump contour and jump matrices for the $\widehat{\Psi} \mathrm{R}-\mathrm{H}$ problem

Thus, $\operatorname{det} \Psi_{0}(\zeta)$ is analytic in $\mathbb{C}$, and so $\operatorname{det} \Psi_{0}(\zeta)=1$ since $\operatorname{det} \Psi_{0}(\zeta)=1+\mathcal{O}\left(\zeta^{-1}\right)$ as $\zeta \rightarrow \infty$. Now using (171), we have $\operatorname{tr} C_{1}=0$. We denote the elements of the matrix $C_{1}$ by

$$
C_{1}(x)=\left(\begin{array}{cc}
q(x) & r(x) \\
t(x) & -q(x)
\end{array}\right)
$$

We define two functions $v$ and $u$ in terms of the matrix elements of $C_{1}$ by setting

$$
v(x)=\frac{\alpha_{0}+\beta_{0}}{2}-q(x)-x r(x) t(x)
$$

and

$$
u(x)=1+\frac{x t}{\left(2 \beta_{0}+1-x\right) t(x)+x t^{\prime}(x)} .
$$

By [3, Section 4.3], the following solution to the Painlevé V equation plays a crucial role in describing transition asymptotics:

$$
\sigma(x)=\int_{x}^{+\infty} v(\xi) d \xi
$$

which is well defined because of the asymptotic behavior of $v$ given as

$$
v(x)= \begin{cases}-\frac{\alpha_{0}^{2}-\beta_{0}^{2}}{2 \alpha_{0}}\left(1-\left(2 \alpha_{0}+1\right) x^{2 \alpha_{0}} C\left(\alpha_{0}, \beta_{0}\right)\right)(1+\mathcal{O}(x)), & x \rightarrow 0,2 \alpha_{0} \notin \mathbb{Z}, \\ \mathcal{O}(1)+\mathcal{O}\left(x^{2 \alpha_{0}}\right)+\mathcal{O}\left(x^{2 \alpha_{0}} \log x\right), & x \rightarrow 0,2 \alpha_{0} \in \mathbb{Z}, \\ -x^{-1+2 \alpha_{0}} e^{-x} \frac{1}{\Gamma\left(\alpha_{0}-\beta_{0}\right) \Gamma\left(\alpha_{0}+\beta_{0}\right)}(1+\mathcal{O}(1 / x)), & x \rightarrow+\infty,\end{cases}
$$

where $C\left(\alpha_{0}, \beta_{0}\right)$ is defined in (46).

Let us denote by $\Psi_{0, \mathrm{I}}, \ldots, \Psi_{0, \mathrm{~V}}$ the analytic continuation of $\Psi_{0}$ from the relevant sectors $\mathrm{I}, \ldots, \mathrm{V}$ in Figure 8 to $\mathbb{C} \backslash[0,+\infty)$. Consider the following function,

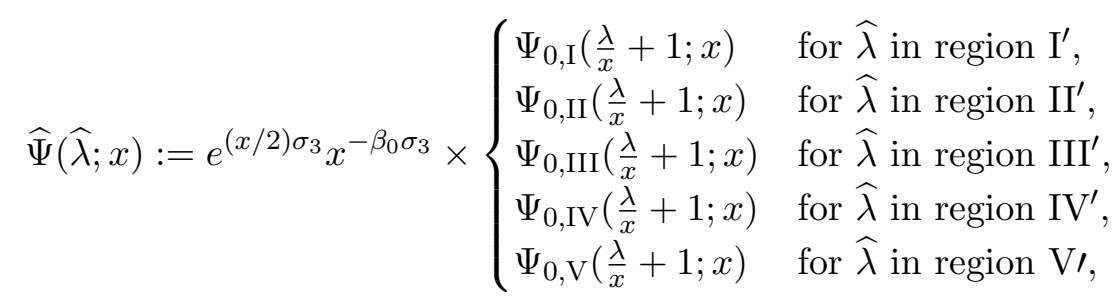

and write $\widehat{\lambda}(z):=\lambda(z)-\frac{x}{2}$. The contour is translated by a half in the $\zeta$-plane and then by realizing that $\zeta(z)=\frac{\lambda(z)}{x}$ (which is not the same as in the R-H problem for $\Psi_{1}$ ), it is transformed into a 
contour in the $\widehat{\lambda}$-plane (see Figure 9 and compare with Figure 8). Observe that the intersection of the contour lines is now at $\widehat{\lambda}=0$ as opposed to $\zeta=\frac{1}{2}$, i.e. $\lambda=\frac{x}{2}$.

The following R-H problem for $\widehat{\Psi}$ follows directly from the $\Psi_{0}$ problem $\left(\Psi_{0} 1\right)-\left(\Psi_{0} 4\right)$.

Riemann-Hilbert Problem for $\widehat{\Psi}$

$(\widehat{\Psi} 1) \quad \widehat{\Psi}: \mathbb{C} \backslash \widehat{\Gamma} \rightarrow \mathbb{C}^{2 \times 2}$ is analytic. Here $\widehat{\Gamma}:=\cup_{j=1}^{6} \widehat{\Gamma}_{j}$ and

$$
\begin{array}{r}
\widehat{\Gamma}_{1}=e^{i \pi / 4} \mathbb{R}^{+}, \quad \widehat{\Gamma}_{2}=e^{3 i \pi / 4} \mathbb{R}^{+}, \quad \widehat{\Gamma}_{3}=e^{5 i \pi / 4} \mathbb{R}^{+}, \\
\widehat{\Gamma}_{4}=e^{7 i \pi / 4} \mathbb{R}^{+}, \quad \widehat{\Gamma}_{5}=\mathbb{R}^{+}, \quad \widehat{\Gamma}_{6}=(-x, 0) .
\end{array}
$$

$(\widehat{\Psi} 2) \quad \widehat{\Psi}$ has continuous boundary values on $\widehat{\Gamma} \backslash\{-x, 0\}$, and they are related by the following jump conditions,

$(\widehat{\Psi} 3) \quad \widehat{\Psi}_{0}$ has the following asymptotic behavior as $\widehat{\lambda} \rightarrow \infty$,

$$
\widehat{\Psi}(\widehat{\lambda})=\left(I+\mathcal{O}\left(\widehat{\lambda}^{-1}\right)\right) \widehat{\lambda}^{-\beta \sigma_{3}} e^{-(1 / 2) \widehat{\lambda} \sigma_{3}} .
$$

$(\widehat{\Psi} 4) \quad \widehat{\Psi}$ has the following asymptotic behavior close to the following points,

$$
\begin{aligned}
& \widehat{\Psi}(\widehat{\lambda})=\mathcal{O}\left(\begin{array}{ll}
|\widehat{\lambda}+x|^{\left(\alpha_{0}-\beta_{0}\right) / 2} & |\widehat{\lambda}+x|^{-\left(\alpha_{0}-\beta_{0}\right) / 2} \\
|\widehat{\lambda}+x|^{\left(\alpha_{0}-\beta_{0}\right) / 2} & |\widehat{\lambda}+x|^{-\left(\alpha_{0}-\beta_{0}\right) / 2}
\end{array}\right), \quad \text { as } \widehat{\lambda} \rightarrow-x, \\
& \widehat{\Psi}(\widehat{\lambda})=\mathcal{O}\left(\begin{array}{ll}
|\widehat{\lambda}|^{-\left(\alpha_{0}+\beta_{0}\right) / 2} & |\widehat{\lambda}|^{\left(\alpha_{0}+\beta_{0}\right) / 2} \\
|\hat{\lambda}|^{-\left(\alpha_{0}+\beta_{0}\right) / 2} & |\widehat{\lambda}|^{\left(\alpha_{0}+\beta_{0}\right) / 2}
\end{array}\right), \quad \text { as } \widehat{\lambda} \rightarrow 0, \widehat{\lambda} \in \mathrm{I}^{\prime}, \mathrm{V}^{\prime},
\end{aligned}
$$

and for $\hat{\lambda}$ in other sectors we apply the appropriate jump conditions to 187 .

As in [3], we use steepest descent techniques, and in particular, for small values of $x$, we construct a global and a local parametrix, match them on the boundary of a small neighborhood of $\hat{\lambda}=0$, denoted by $U_{\varepsilon}$ (which contains also $[-x, 0]$ ), and show the final R-H problem is a small norm R-H problem, and hence solvable. We summarize the key points below.

Riemann-Hilbert Problem for $M$ (Global Parametrix for the $\widehat{\Psi} R$-H problem)

(M1) $M: \mathbb{C} \backslash\left(\cup_{j=1}^{5} \widehat{\Gamma}_{j}\right) \rightarrow \mathbb{C}^{2 \times 2}$ is analytic. 
(M2) $M$ has continuous boundary values on $\left(\cup_{j=1}^{5} \widehat{\Gamma}_{j}\right) \backslash\{0\}$ related by the following jump conditions:

$$
\begin{gathered}
M_{+}(\widehat{\lambda})=M_{-}(\widehat{\lambda})\left(\begin{array}{cc}
1 & e^{\pi i\left(\alpha_{0}-\beta_{0}\right)} \\
0 & 1
\end{array}\right) \quad \text { for } \widehat{\lambda} \in \widehat{\Gamma}_{1}, \\
M_{+}(\widehat{\lambda})=M_{-}(\widehat{\lambda})\left(\begin{array}{cc}
1 & 0 \\
-e^{-\pi i\left(\alpha_{0}-\beta_{0}\right)} & 1
\end{array}\right) \quad \text { for } \widehat{\lambda} \in \widehat{\Gamma}_{2}, \\
M_{+}(\widehat{\lambda})=M_{-}(\widehat{\lambda})\left(\begin{array}{cc}
1 & 0 \\
e^{\pi i\left(\alpha_{0}-\beta_{0}\right)} & 1
\end{array}\right) \quad \text { for } \widehat{\lambda} \in \widehat{\Gamma}_{3}, \\
M_{+}(\widehat{\lambda})=M_{-}(\widehat{\lambda})\left(\begin{array}{cc}
1 & -e^{-\pi i\left(\alpha_{0}-\beta_{0}\right)} \\
0 & 1
\end{array}\right) \quad \text { for } \widehat{\lambda} \in \widehat{\Gamma}_{4}, \\
M_{+}(\widehat{\lambda})=M_{-}(\widehat{\lambda}) e^{2 \pi i \beta_{0} \sigma_{3}} \quad \text { for } \widehat{\lambda} \in \widehat{\Gamma}_{5},
\end{gathered}
$$

(M3) $M$ has the following behavior at infinity,

$$
M(\widehat{\lambda})=\left(I+\mathcal{O}\left(\widehat{\lambda}^{-1}\right)\right) \widehat{\lambda}^{-\beta_{0} \sigma_{3}} e^{-(1 / 2) \widehat{\lambda} \sigma_{3}} \quad \text { as } \widehat{\lambda} \rightarrow \infty .
$$

The problem is explicitly solvable in terms of the confluent hypergeometric function $\psi$ defined via (115). We give the solution as it was presented in [3, Section 4.2.1], but only for sector I' (compare with the analysis performed in Section 7.4), details of other sectors can be found in [3], or by applying the appropriate jumps. We define the following matrix-valued function,

$$
\begin{aligned}
& H(\widehat{\lambda}):=\left(\begin{array}{cc}
e^{-i \pi\left(2 \beta_{0}+\alpha_{0}\right)} & 0 \\
0 & e^{i \pi\left(\beta_{0}+2 \alpha_{0}\right)}
\end{array}\right) e^{-(i \pi / 2) \alpha_{0} \sigma_{3}} \\
& \times\left(\begin{array}{c}
\hat{\lambda}^{\alpha_{0}} \psi\left(\alpha_{0}+\beta_{0}, 1+2 \alpha_{0}, \widehat{\lambda}\right) e^{i \pi\left(2 \beta_{0}+\alpha_{0}\right)} \\
\widehat{\lambda}^{-\alpha_{0}} \psi\left(1-\alpha_{0}+\beta_{0}, 1-2 \alpha_{0}, \widehat{\lambda}\right) e^{i \pi\left(\beta_{0}-3 \alpha_{0}\right)} \frac{\Gamma\left(1+\alpha_{0}+\beta_{0}\right)}{\Gamma\left(\alpha_{0}-\beta_{0}\right)}
\end{array}\right. \\
& \left.\widehat{\lambda}^{\alpha_{0}} \psi\left(1+\alpha_{0}-\beta_{0}, 1+2 \alpha_{0}, e^{-i \pi} \hat{\lambda}\right) e^{i \pi\left(\beta_{0}+\alpha_{0}\right)} \frac{\Gamma\left(1+\alpha_{0}-\beta_{0}\right)}{\Gamma\left(\alpha_{0}+\beta_{0}\right)}\right) \\
& \widehat{\lambda}^{-\alpha_{0}} \psi\left(-\alpha_{0}-\beta_{0}, 1-2 \alpha_{0}, e^{-i \pi} \widehat{\lambda}\right) e^{i \pi \alpha_{0}} \\
& \times e^{\left(i \pi \alpha_{0} / 2\right) \sigma_{3}} e^{-\widehat{\lambda} \sigma_{3} / 2}, \quad \alpha_{0} \pm \beta_{0} \neq-1,-2, \ldots,
\end{aligned}
$$

where $\psi(a, b, x)$ is the confluent hypergeometric function (see 115$)$ and $(116)$ for some of its properties), and $\Gamma(x)$ is the Euler's $\Gamma$-function. The solution to the $\mathrm{R}-\mathrm{H}$ problem for $M$ in the sector $\mathrm{I}^{\prime}$ is then given by,

$$
M(\widehat{\lambda})=M_{1}(\widehat{\lambda}):=H(\widehat{\lambda})\left(\begin{array}{cc}
1 & -e^{i \pi\left(\alpha_{0}-\beta_{0}\right)} \\
0 & 1
\end{array}\right), \quad \text { for } 0<\arg \widehat{\lambda}<\frac{\pi}{4},
$$

and we denote the matrix elements of $M(\widehat{\lambda})$ by $M_{i j}, i, j=1,2$ for future use. Also, in [3], it is shown that for $\hat{\lambda} \in \partial U_{\varepsilon}$,

$$
\begin{aligned}
P(\widehat{\lambda} ; x) M(\widehat{\lambda})^{-1} & = \begin{cases}I+\mathcal{O}(x)+\mathcal{O}\left(x^{1+2 \alpha_{0}}\right), & \text { as } x \rightarrow 0, \text { if } 2 \alpha_{0} \notin \mathbb{Z}, \\
I+\mathcal{O}(x \log x), & \text { as } x \rightarrow 0, \text { if } 2 \alpha_{0} \in \mathbb{Z} .\end{cases} \\
& =I+o(1) \quad \text { as } x \rightarrow 0,
\end{aligned}
$$

which makes the following final R-H problem small-norm for sufficiently small $x$.

Riemann-Hilbert problem for $\widehat{R}$

$(\widehat{R} 1) \widehat{R}: \mathbb{C} \backslash \partial U_{\varepsilon} \rightarrow \mathbb{C}^{2 \times 2}$ is analytic. 


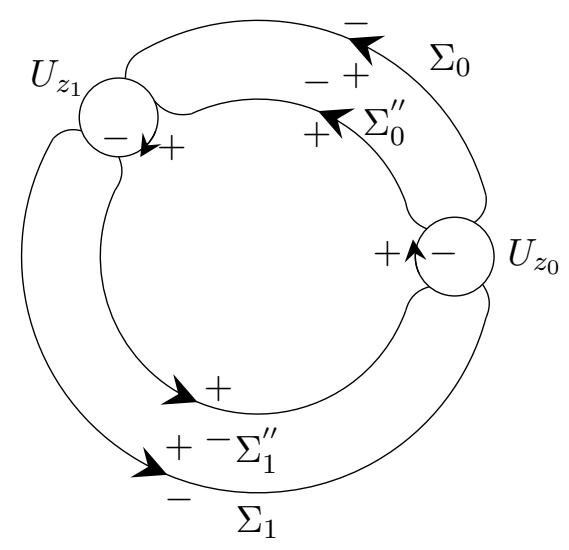

Figure 10. Contour $\Gamma=\cup_{j=0}^{1} \partial U_{z_{j}} \cup_{j=0}^{1} \Sigma_{j} \cup_{j=0}^{1} \Sigma_{j}^{\prime \prime}$ for the $R$ and $\tilde{R} \mathrm{R}-\mathrm{H}$ problem.

( $\widehat{R} 2) \widehat{R}$ has the following jump condition,

$$
\widehat{R}_{+}(\widehat{\lambda})=\widehat{R}_{-}(\widehat{\lambda}) J(\widehat{\lambda}), \quad \text { for } \widehat{\lambda} \in \partial U_{\varepsilon},
$$

where $J(\widehat{\lambda})=P(\widehat{\lambda}) M(\widehat{\lambda})^{-1}$.

$(\widehat{R} 3) \widehat{R}(\widehat{\lambda})=I+\mathcal{O}\left(\widehat{\lambda}^{-1}\right)$ as $\widehat{\lambda} \rightarrow \infty$.

The solvability of this problem implies through the invertible transformations $\Psi_{0} \rightarrow \widehat{\Psi} \rightarrow \widehat{R}$ and $\Psi_{0} \rightarrow \Phi$ that the R-H problems for $\Phi$ and $\Psi_{0}$ are solvable for $0<x<\delta$. Further, from the fact that $J(\widehat{\lambda})=I+o(1)$ and $(\widehat{R} 2)$, it follows that $\widehat{R}(\widehat{\lambda})=1+o(1)$ uniformly for $\widehat{\lambda} \in \mathbb{C} \backslash U_{\varepsilon}$ as $x \rightarrow 0$. This holds at infinity, and so $\widehat{R}(\widehat{\lambda})=I+\mathcal{O}\left(\widehat{\lambda}^{-1}\right)$ as $\widehat{\lambda} \rightarrow \infty$ uniformly for small $x$.

The following function solves the R-H problem for $\widehat{R}$ above,

$$
\widehat{R}(\widehat{\lambda})= \begin{cases}\widehat{\Psi}(\widehat{\lambda}) M(\widehat{\lambda})^{-1} & \text { for } \widehat{\lambda} \in \mathbb{C} \backslash U_{\varepsilon}, \\ \widehat{\Psi}(\widehat{\lambda}) P(\widehat{\lambda})^{-1} & \text { for } \widehat{\lambda} \in U_{\varepsilon} .\end{cases}
$$

7.8. Final Riemann-Hilbert Problem. We assume that $\alpha_{j} \pm \beta_{j} \neq-1,-2, \ldots$ for $j=0,1$. Let

$$
R(z)= \begin{cases}S(z) N^{-1}(z), & z \in \mathbb{C} \backslash\left\{U_{z_{1}} \cup U_{z_{0}} \cup \Gamma\right\}, \\ S(z) P_{z_{1}}^{-1}(z), & z \in U_{z_{1}} \\ S(z) P_{z_{0}}^{-1}(z), & z \in U_{z_{0}}\end{cases}
$$

As mentioned in Sections 7.4 and 7.6. $S P_{z_{0}}^{-1}$ and $S P_{z_{1}}^{-1}$ are analytic in the neighborhoods $U_{z_{0}}$ and $U_{z_{1}}$ respectively. The function $R$ satisfies the following Riemann-Hilbert problem, for which the contour $\Gamma$ is defined in Figure 10 .

$R: \mathbb{C} \backslash \Gamma \rightarrow \mathbb{C}^{2 \times 2}$ is analytic.

$R$ satisfies the following jump conditions:

$$
R_{+}(z)=R_{-}(z) J_{R}(z) \quad \text { for } z \in \Gamma .
$$

The jump matrices are defined by

$$
J_{R}(z)=N(z) J_{k}(z) N(z)^{-1} \quad \text { with }\left\{\begin{array}{l}
k=1 \text { for } z \in \Sigma:=\Sigma_{0} \cup \Sigma_{1}, \\
k=2 \text { for } z \in \Sigma^{\prime \prime}:=\Sigma_{0}^{\prime \prime} \cup \Sigma_{1}^{\prime \prime},
\end{array}\right.
$$


and

$$
\begin{gathered}
J_{R}(z)=P_{z_{1}}(z) N^{-1}(z) \text { for } z \in \partial U_{z_{1}}, \\
J_{R}(z)=P_{z_{0}}(z) N^{-1}(z) \text { for } z \in \partial U_{z_{0}} . \\
\text { Recall from }(74) \text { that } J_{1}(z)=\left(\begin{array}{cc}
1 & 0 \\
z^{-n} f_{t}(z)^{-1} & 1
\end{array}\right), J_{2}(z)=\left(\begin{array}{cc}
1 & 0 \\
z^{n} f_{t}(z)^{-1} & 1
\end{array}\right) . \\
R(z)=I+\mathcal{O}(1 / z) \text { as } z \rightarrow \infty .
\end{gathered}
$$

To find the solution of the R-H problem for $R$, we look at the asymptotic behavior of the jump matrices as $n \rightarrow \infty$. The jump matrices have $I+o(1)$ behavior at infinity, the problem is a so-called small norm problem and solution is given in terms of a Neumann series (see [9, Theorem 7.8]).

The jump matrices $J_{R}$ on $\Sigma$ and $\Sigma^{\prime \prime}$ can be estimated uniformly as $I+\mathcal{O}\left(e^{-\varepsilon n}\right)$, for a positive constant $\varepsilon$ using the $J_{k}$ above (80) and (81) for $0<t<t_{0}$ and $x=2 n t$ bounded away from the set $\left\{x_{1}, \ldots, x_{k}\right\}$. Thus these jump matrices already behave the way we require.

On the contour $\partial U_{z_{1}}$, the jump matrix admits a uniform expansion in inverse powers of $n$, conjugated by $n^{\beta_{1} \sigma_{3}} z_{1}^{-n \sigma_{3} / 2}$,

$$
J_{R}(z)=I+\Delta_{1}(z)+\Delta_{2}(z)+\cdots+\Delta_{k}(z)+\Delta_{k+1}^{(r)}(z) \quad \text { for } z \in \partial U_{z_{1}} .
$$

For each $k \in \mathbb{N}$ and $z \in \partial U_{z_{1}}$, both $\Delta_{k}(z)$ and $\Delta_{k}^{(r)}(z)$ are of the form

$$
z_{1}^{\sigma_{3} n / 2} n^{-\sigma_{3} \beta_{1}} \mathcal{O}\left(1 / n^{k}\right) n^{\sigma_{3} \beta_{1}} z_{1}^{-\sigma_{3} n / 2} .
$$

To obtain the solution, we require that $J_{R}=I+o(1)$. For $z \in \partial U_{z_{0}}$, we use (133) to get

$$
P_{z_{0}}(z) N(z)^{-1}=I+n^{-\beta_{0} \sigma_{3}} \mathcal{O}\left(n^{-1}\right) n^{\beta_{0} \sigma_{3}}=I+\Delta_{1}(z)+\cdots+\Delta_{k}(z)+\Delta_{k+1}(z),
$$

which also needs to be of order $I+o(1)$. Combining the two cases, we have

$$
n^{2 \max _{j}\left|\operatorname{Re} \beta_{j}\right|-1}=o(1)
$$

and so $-\frac{1}{2}<\operatorname{Re} \beta_{j}<\frac{1}{2}$ for $j=0,1$.

However, we need not stipulate that $\operatorname{Re} \beta_{j} \in\left(-\frac{1}{2}, \frac{1}{2}\right)$. It is possible to find a solution for $\operatorname{Re} \beta_{j} \in\left(q-\frac{1}{2}, q+\frac{1}{2}\right)$, for some $q \in \mathbb{R}$; i.e. the more general condition when $\||\beta \||<1$. To accommodate these cases, we consider the following transformation of the R-H problem for $R$ which was used in [6, Equation (4.63)]:

$$
\tilde{R}(z)=n^{\omega \sigma_{3}} R(z) n^{-\omega \sigma_{3}},
$$

where

$$
\omega=\frac{1}{2}\left(\min _{j=0,1} \operatorname{Re} \beta_{j}+\max _{j=0,1} \operatorname{Re} \beta_{j}\right) .
$$

This transformation moves all $\beta_{j}$ into the strip $\left(-\frac{1}{2}, \frac{1}{2}\right)$ making the above asymptotics of the jump matrices of the order $I+o(1)$. Note that the $\beta_{j}$ are moved only in the conjugation by $n^{\beta_{j}}$, and not in the actual Fisher-Hartwig symbol $f(z ; t)$.

The transformation affects the jump conditions of the problem for $R(z)$ as follows:

$$
\begin{aligned}
\tilde{R}_{+}(z) & =n^{\omega \sigma_{3}} R_{+}(z) n^{-\omega \sigma_{3}}=n^{\omega \sigma_{3}} R_{-}(z) J_{R}(z) n^{-\omega \sigma_{3}} \\
& =n^{\omega \sigma_{3}} R_{-}(z) n^{-\omega \sigma_{3}} n^{\omega \sigma_{3}} J_{R}(z) n^{-\omega \sigma_{3}}=\tilde{R}_{-}(z) n^{\omega \sigma_{3}} J_{R}(z) n^{-\omega \sigma_{3}} .
\end{aligned}
$$

The asymptotic behavior of the jump matrices on $\Sigma$ and $\Sigma^{\prime \prime}$ remains unchanged by the transformation, $\tilde{J}_{R}(z)=I+\mathcal{O}\left(e^{-\varepsilon n}\right)$, but with a different $\varepsilon$. 
The jump matrices on $\partial U_{z_{0}}$ and $\partial U_{z_{1}}$ are now of the form

$$
I+n^{\omega \sigma_{3}} \Delta_{1}(z) n^{-\omega \sigma_{3}}+n^{\omega \sigma_{3}} \Delta_{2}(z) n^{-\omega \sigma_{3}}+\cdots+n^{\omega \sigma_{3}} \Delta_{k}(z) n^{-\omega \sigma_{3}}+n^{\omega \sigma_{3}} \Delta_{k+1}^{(r)}(z) n^{-\omega \sigma_{3}},
$$

and the order of each of the terms is $\mathcal{O}\left(n^{2 \max _{j}\left|\beta_{j}-\omega\right|-k}\right)$, which behaves as $I+o(1)$ since $-\frac{1}{2}<$ $\beta_{0}-\omega<\frac{1}{2}$. This means that we can find the solution to the problem for $\tilde{R}$ for $\beta$-parameters in the range $\operatorname{Re} \beta_{j} \in(q-1 / 2, q+1 / 2)$ for any $q \in \mathbb{R}$.

Now that we have all the jump matrices of the right order, we can use [9, Theorem 7.8] to get the Neumann series solution of the problem $\tilde{R}$ as follows:

$$
\tilde{R}(z)=I+\sum_{p=1}^{k} \tilde{R}_{p}(z)+\tilde{R}_{k+1}^{(r)}(z) .
$$

Each $\tilde{R}_{p}$, is computed recursively via separate, additive $\mathrm{R}-\mathrm{H}$ problems. The conditions are that each $\tilde{R}_{p}$ is analytic outside $\partial U=\partial U_{z_{0}} \cup \partial U_{z_{1}}, \tilde{R}_{p}(z) \rightarrow 0$ as $z \rightarrow \infty$ for all $p$ and satisfies the following jump condition:

$$
\tilde{R}_{p,+}(z)=\tilde{R}_{p,-}(z)+\sum_{i=1}^{p} \tilde{R}_{p-i,-}(z) n^{\omega \sigma_{3}} \Delta_{i}(z) n^{-\omega \sigma_{3}}
$$

where we set $\tilde{R}_{0}(z)=I$. The first R-H problem for $\tilde{R}_{1}$ satisfies the following conditions:

$\left(\tilde{\mathrm{R}}_{1} 1\right) \quad \tilde{R}_{1}: \mathbb{C} \backslash \partial U \rightarrow \mathbb{C}^{2 \times 2}$ is analytic.

$\left(\tilde{\mathrm{R}}_{1} 2\right) \quad \tilde{R}_{1}$ satisfies the following jump condition,

$$
\tilde{R}_{1,+}(z)=\tilde{R}_{1,-}(z)+n^{\omega \sigma_{3}} \Delta_{1}(z) n^{-\omega \sigma_{3}} \quad \text { for } z \in \partial U .
$$

$\left(\tilde{\mathrm{R}}_{1} 3\right) \quad \tilde{R}_{1}(z) \rightarrow 0$ as $z \rightarrow \infty$.

First, we recall the transformation (207), and write

$$
R_{p}(z)=n^{-\omega \sigma_{3}} \tilde{R}_{p}(z) n^{\omega \sigma_{3}}, \quad R_{p}^{(r)}(z)=n^{-\omega \sigma_{3}} \tilde{R}_{p}^{(r)}(z) n^{\omega \sigma_{3}} .
$$

Using the Plemelj formulas and the residue theorem, we obtain the following solution to this additive R-H problem:

$$
\begin{aligned}
R_{1}(z) & =\frac{1}{2 \pi i} \int_{\partial U} \frac{\Delta_{1}(x) d x}{x-z} \\
& = \begin{cases}\frac{A_{1}}{z-z_{1}}+\frac{A_{e} t}{z-e^{t}}+\frac{A_{e}-t}{z-e^{-t}}, & z \in \mathbb{C} \backslash \cup_{j=0}^{1} U_{z_{j}}, \\
\frac{A_{1}}{z-z_{1}}+\frac{A_{e^{t}}}{z-e^{t}}+\frac{A_{e}-t}{z-e^{-t}}-\Delta_{1}(z), & z \in U_{z_{j}}, j=0,1,\end{cases}
\end{aligned}
$$

where the contours in the integral are oriented in the negative direction (as seen in Figure 10) and $A_{1}, A_{e^{ \pm t}}$ are the coefficients in the Laurent expansion of $\Delta_{1}$,

$$
\Delta_{1}(z)=\frac{A_{k}}{z-z_{1}}+B_{1}+\mathcal{O}\left(z-z_{1}\right), \quad z \rightarrow z_{1},
$$

and

$$
\Delta_{1}(z)=\frac{A_{e^{ \pm t}}}{z-e^{ \pm t}}+B_{1}+\mathcal{O}\left(z-e^{ \pm t}\right), \quad z \rightarrow e^{ \pm t} .
$$

The coefficients $A_{1}, A_{e^{ \pm t}}$ are given below and $B_{1}, B_{e^{ \pm t}}$ can also be computed explicitly if needed. In Sections 7.4 and 7.6 we computed the 12 entries of $\Delta_{1}$ of each parametrix at the points $z_{1}, e^{-t}$ 
and $e^{t}$, they are given by (106), (159) and (160). Using those together with (107), (158) and (157) respectively, we obtain the 12 elements of the matrices $A_{z_{1}}, A_{e^{-t}}, A_{e^{t}}$,

$$
\begin{aligned}
A_{z_{1}}= & \frac{z_{1}}{n} z_{1}^{n} e^{V_{0}}\left(1-z_{1} e^{t}\right)^{\left(\alpha_{0}+\beta_{0}\right)}\left(1-e^{-t} z_{1}^{-1}\right)^{-\left(\alpha_{0}-\beta_{0}\right)} \exp \left\{\sum_{k=1}^{\infty} V_{k} z_{1}^{k}\right\} \exp \left\{-\sum_{k=1}^{\infty} V_{-k} z_{k}^{-k}\right\} \\
& \times e^{t\left(\alpha_{0}+\beta_{0}\right)} n^{-2 \beta_{1}} \frac{\Gamma\left(1+\alpha_{1}+\beta_{1}\right)}{\Gamma\left(\alpha_{1}-\beta_{1}\right)}(1+\mathcal{O}(u))
\end{aligned}
$$

$$
\begin{aligned}
A_{e^{-t}}= & \frac{e^{-t}}{n}\left(1-z_{1}^{-1} e^{-t}\right)^{2 \beta_{1}}\left(1-e^{-2 t}\right)^{\alpha_{0}+\beta_{0}} e^{V_{0}} \exp \left\{\sum_{k=1}^{\infty} V_{k} e^{-t k}\right\} \exp \left\{-\sum_{k=1}^{\infty} V_{-k} e^{t k}\right\} \\
& \times e^{t\left(\alpha_{0}+\beta_{0}\right)} e^{-t\left(\alpha_{1}-\beta_{1}\right)} e^{-i \pi\left(\alpha_{1}-\beta_{1}\right)} z_{1}^{-\left(\alpha_{1}-\beta_{1}\right)}(2 t)^{-\left(\alpha_{0}+\beta_{0}\right)} \frac{n^{-2 \beta_{0}}}{\Gamma\left(\alpha_{0}-\beta_{0}\right)} K(2 n t)\left(1+\mathcal{O}\left(n^{-1}\right)\right), \\
A_{e^{t}}= & \frac{e^{t}}{n}\left(1-z_{1} e^{-t}\right)^{2 \beta_{1}}\left(1-e^{-2 t}\right)^{-\left(\alpha_{0}-\beta_{0}\right)} e^{V_{0}} \exp \left\{\sum_{k=1}^{\infty} V_{k} e^{t k}\right\} \exp \left\{-\sum_{k=1}^{\infty} V_{-k} e^{-t k}\right\} \\
& \times e^{t\left(\alpha_{0}+\beta_{0}\right)} e^{t\left(\alpha_{1}+\beta_{1}\right)} e^{-i \pi\left(\alpha_{1}+\beta_{1}\right)} z_{1}^{-\left(\alpha_{1}+\beta_{1}\right)}(2 t)^{\alpha_{0}-\beta_{0}} \frac{n^{-2 \beta_{0}}}{\Gamma\left(\alpha_{0}-\beta_{0}\right)} K(2 n t)\left(1+\mathcal{O}\left(n^{-1}\right)\right) .
\end{aligned}
$$

Next we look at the R-H problem for $\tilde{R}_{2}$ :

$\left(\tilde{R}_{2} 1\right) \quad \tilde{R}_{2}: \mathbb{C} \backslash \partial U \rightarrow \mathbb{C}^{2 \times 2}$ is analytic.

$\left(\tilde{\mathrm{R}}_{2} 2\right) \quad \tilde{R}_{2}$ satisfies the following jump condition:

$$
\tilde{R}_{2,+}(z)=\tilde{R}_{2,-}(z)+\tilde{R}_{1,-}(z) n^{\omega \sigma_{3}} \Delta_{1}(z) n^{-\omega \sigma_{3}}+n^{\omega \sigma_{3}} \Delta_{2}(z) n^{-\omega \sigma_{3}} \quad \text { for } z \in \partial U
$$

$\left(\tilde{\mathrm{R}}_{2} 3\right) \quad \tilde{R}_{2}(z) \rightarrow 0$ as $z \rightarrow \infty$.

Using again the Plemelj formulas, we see that the solutions are given by evaluating the integral

$$
\tilde{R}_{2}(z)=\frac{1}{2 \pi i} \int_{\partial U}\left(\tilde{R}_{1,-}(z) n^{\omega \sigma_{3}} \Delta_{1}(z) n^{-\omega \sigma_{3}}+n^{\omega \sigma_{3}} \Delta_{2}(z) n^{-\omega \sigma_{3}}\right) \frac{d x}{x-z},
$$

where each $\Delta_{k}(z)=\mathcal{O}\left(n^{2 \max _{j}\left|\operatorname{Re} \beta_{j}\right|-k}\right)$ as in (4.66) and (4.74) of [6]. Therefore, since we are using the same transformation, we have $\tilde{R}_{k+1}^{(r)}(z)=\mathcal{O}\left(\left|\tilde{R}_{k+1}(z)\right|\right)+\mathcal{O}\left(\left|\tilde{R}_{k+2}(z)\right|\right)$ and

$$
R_{3}^{(r)}(z)=\left(\begin{array}{cc}
\mathcal{O}(\delta / n)+\mathcal{O}\left(\delta^{2}\right) & \mathcal{O}\left(\delta \max _{k} \frac{n^{-2 \operatorname{Re} \beta_{k}}}{n}\right) \\
\mathcal{O}\left(\delta \max _{k} \frac{n^{2} \operatorname{Re} \beta_{k}}{n}\right) & \mathcal{O}(\delta / n)+\mathcal{O}\left(\delta^{2}\right)
\end{array}\right)
$$

where

$$
\delta=\max _{j, k} n^{2 \operatorname{Re}\left(\beta_{j}-\beta_{k}-1\right)} .
$$

\section{Proof of Theorem 5}

We first assume that $\alpha_{0} \in \mathbb{R}$ and $\beta_{0} \in i \mathbb{R}$; for the general case, see Remark 12 below. Now, to obtain the asymptotics of the Toeplitz determinant if $\|\beta\|<1$, we need to go through reverse transformations. We are only interested in the solution near $z=e^{t}$ and $z=e^{-t}$ because this is what the differential identity (60) calls for. In Section 7.8 we solved the final R-H problem, providing details for the first terms in the series $R(z)=I+\sum_{p=1}^{k} R_{p}(z)+R_{k+1}^{(r)}(z)$. These details will be used to find asymptotics for the determinant if the seminorm $\|\beta\|=1$. Here however, we only 
require that $\tilde{R}(z)=I+\mathcal{O}\left(n^{-1}\right)$ as $n \rightarrow \infty$ uniformly for $z \in \mathbb{C} \backslash \Gamma$, which follows from the solution obtained in Section 7.8. Following the transformations, we obtain that

$$
\begin{aligned}
Y(z) & = \begin{cases}n^{-\omega \sigma_{3}}\left(I+\mathcal{O}\left(n^{-1}\right)\right) n^{\omega \sigma_{3}} P_{z_{0}}(z) z^{n \sigma_{3}}, & z=e^{t}, \\
n^{-\omega \sigma_{3}}\left(I+\mathcal{O}\left(n^{-1}\right)\right) n^{\omega \sigma_{3}} P_{z_{0}}(z), & z=e^{-t},\end{cases} \\
& = \begin{cases}n^{-\omega \sigma_{3}}\left(I+\mathcal{O}\left(n^{-1}\right)\right) n^{\omega \sigma_{3}} D(z)^{\sigma_{3}} W(z)^{-1} \Phi(z) W(z) z^{n \sigma_{3}}, & z=e^{t}, \\
n^{-\omega \sigma_{3}}\left(I+\mathcal{O}\left(n^{-1}\right)\right) n^{\omega \sigma_{3}} D(z)^{\sigma_{3}}\left(\begin{array}{cc}
0 & 1 \\
-1 & 0
\end{array}\right) W(z)^{-1} \Phi(z) W(z), & z=e^{-t} .\end{cases}
\end{aligned}
$$

It follows from the R-H problem for $\tilde{R}(z)$ that the asymptotics for $Y(z)$ as $n \rightarrow \infty$ are uniform for $0<t<t_{0}$ for a sufficiently small $t_{0}$, as long as $2 n t$ remains bounded away from the set of numbers $\left\{x_{0}, x_{1}, \ldots, x_{k}\right\}$.

It now remains to substitute the asymptotics into the differential identity. We need to evaluate $Y^{-1} Y_{z}^{\prime}$ at the points $z=e^{t}$ and $z=e^{-t}$. Differentiating the expressions in 222 we obtain,

$$
Y^{-1} Y_{z}^{\prime}= \begin{cases}\frac{n \sigma_{3}}{z}+z^{-n \sigma_{3}} P^{-1} P_{z}^{\prime} z^{n \sigma_{3}}+ & \\ +z^{-n \sigma_{3}} P^{-1}(z) n^{-\omega \sigma_{3}}\left(I+\mathcal{O}\left(n^{-1}\right)\right)^{-1} \mathcal{O}\left(n^{-1}\right)_{z}^{\prime} n^{\omega \sigma_{3}} P(z) z^{n \sigma_{3}}, & \text { near } e^{t}, \\ P^{-1} P_{z}^{\prime}+P^{-1}(z) n^{-\omega \sigma_{3}}\left(I+\mathcal{O}\left(n^{-1}\right)\right)^{-1} n^{\omega \sigma_{3}} P(z), & \text { near } e^{-t} .\end{cases}
$$

We define a function $A$ by

$$
A(z)= \begin{cases}G(\lambda(z))^{-1 / 2} z^{n / 2} f_{t}(z)^{1 / 2}, & \text { for }|z|>1 \\ -G(\lambda(z))^{-1 / 2} z^{n / 2} f_{t}(z)^{-1 / 2}, & \text { for }|z|<1\end{cases}
$$

Then

$$
W(z)= \begin{cases}A(z)^{\sigma_{3}} \sigma_{1}, & \text { for }|z|>1 \\ A(z)^{\sigma_{3}} \sigma_{3}, & \text { for }|z|<1\end{cases}
$$

We now find, using (223),

$$
\begin{aligned}
& P^{-1} P_{z}^{\prime}= \begin{cases}-\sigma_{3} \frac{A_{z}^{\prime}}{A}+W^{-1} \Phi^{-1} \Phi_{z}^{\prime} W-W^{-1} \Phi^{-1} \sigma_{3} \Phi W\left(\frac{A_{z}^{\prime}}{A}+\frac{D_{z}^{\prime}}{D}\right), & \text { near } e^{t}, \\
\sigma_{3} \frac{A_{z}^{\prime}}{A}+W^{-1} \Phi^{-1} \Phi_{z}^{\prime} W-W^{-1} \Phi^{-1} \sigma_{3} \Phi W\left(\frac{A_{z}^{\prime}}{A}+\frac{D_{z}^{\prime}}{D}\right), & \text { near } e^{-t},\end{cases} \\
& \left(G^{-\frac{1}{2}}(\lambda(z), 2 n t)\right)_{z}^{\prime}=\left[\left(\alpha_{0}-\beta_{0}\right)(n \log (z)+n t)^{-1}-\left(\alpha_{0}+\beta_{0}\right)(n \log (z)-n t)^{-1}-1\right] \\
& \times \frac{1}{2}\left(\frac{n}{z}\right) G(\lambda(z))^{-\frac{1}{2}} \\
& A_{z}^{\prime}(z)=\left[\frac{1}{2}\left(\alpha_{0}-\beta_{0}\right)(n \log (z)+n t)^{-1}-\frac{1}{2}\left(\alpha_{0}+\beta_{0}\right)(n \log (z)-n t)^{-1}\right]\left(\frac{n}{z}\right) A(z) \\
& +\frac{1}{2} f_{z}^{\prime}(z) f^{-1}(z) A(z) \\
& f_{z}^{\prime}(z)=V^{\prime}(z) f_{t}(z)-\left(\alpha_{1}-\beta_{1}\right) z^{-1} f_{t}(z)+2 \alpha_{1}\left(z-z_{1}\right)^{-1} f_{t}(z) \\
& +\left(\alpha_{0}+\beta_{0}\right)\left(z-e^{t}\right)^{-1} f_{t}(z)+\left(\alpha_{0}-\beta_{0}\right)\left(z-e^{-t}\right)^{-1} f_{t}(z)+\left(-\alpha_{0}+\beta_{0}\right) z^{-1} f_{t}(z)
\end{aligned}
$$


We compute the derivative of $\left|z-z_{1}\right|^{2 \alpha}$ near $e^{ \pm t}$ using the function $h_{\alpha_{j}}(z)$ from (84), and get $\left(h_{\alpha_{j}}^{2}\right)_{z}^{\prime}=2\left(h_{\alpha_{j}}\right)_{z}^{\prime} h_{\alpha_{j}}$, where

$$
\left(h_{\alpha_{j}}\right)_{z}^{\prime}(z)=\alpha_{j}\left(z-z_{j}\right)^{-1} h_{\alpha_{j}}(z)-\frac{\alpha_{j}}{2} z^{-1} h_{\alpha_{j}}(z) .
$$

Using (229) and (233),

$$
\frac{A_{z}^{\prime}}{A}(z)= \begin{cases}\frac{\alpha_{0}+\beta_{0}}{4} e^{-t}+\frac{\alpha_{0}-\beta_{0}}{4} e^{-t}\left(\frac{1}{t}+\frac{e^{-t}}{\sinh t}\right)+\frac{1}{2} V_{z}^{\prime}\left(e^{t}\right)-\frac{\alpha_{1}-\beta_{1}}{2} e^{-t}+2 \alpha_{1}\left(e^{t}-z_{1}\right)^{-1} & \text { near } e^{t}, \\ \frac{\alpha_{0}+\beta_{0}}{4} e^{t}\left(\frac{1}{t}+\frac{e^{-t}}{\sinh t}\right)+\frac{\alpha_{0}-\beta_{0}}{4} e^{t}-\frac{1}{2} V_{z}^{\prime}\left(e^{-t}\right)+\frac{\alpha_{1}-\beta_{1}}{2} e^{t}-2 \alpha_{1}\left(e^{-t}-z_{1}\right)^{-1} & \text { near } e^{-t} .\end{cases}
$$

Differentiating (81) gives,

$$
\frac{D_{z}^{\prime}}{D}(z)= \begin{cases}\left(-\alpha_{1}+\beta_{1}\right)\left(\frac{z_{1} e^{-t}}{e^{t}-z_{1}}\right)-\left(\alpha_{0}-\beta_{0}\right) \frac{e^{-2 t}}{\sinh t}-\sum_{k=-\infty}^{-1} k V_{k} e^{t(k-1)} & \text { for } z=e^{t}, \\ \left(\alpha_{1}+\beta_{1}\right) \frac{1}{e^{-t}-z_{1}}-\left(\alpha_{0}+\beta_{0}\right) \frac{1}{2 \sinh t}+\sum_{k=0}^{\infty} k V_{k} e^{-t(k-1)} & \text { for } z=e^{-t} .\end{cases}
$$

Using 227), we have

$$
\left(P^{-1} P_{z}^{\prime}\right)_{22}\left(e^{t}\right)=\frac{A_{z}^{\prime}}{A}\left(e^{t}\right)+\left(\Phi^{-1} \Phi_{z}^{\prime}\right)_{11}\left(e^{t}\right)-\left(\Phi^{-1} \sigma_{3} \Phi_{z}^{\prime}\right)_{11}\left(e^{t}\right)\left[\frac{A_{z}^{\prime}}{A}\left(e^{t}\right)+\frac{D_{z}^{\prime}}{D}\left(e^{t}\right)\right],
$$

and

$$
\left(P^{-1} P_{z}^{\prime}\right)_{22}\left(e^{-t}\right)=-\frac{A_{z}^{\prime}}{A}\left(e^{-t}\right)+\left(\Phi^{-1} \Phi_{z}^{\prime}\right)_{22}\left(e^{-t}\right)-\left(\Phi^{-1} \sigma_{3} \Phi_{z}^{\prime}\right)_{22}\left(e^{-t}\right)\left[\frac{A_{z}^{\prime}}{A}\left(e^{-t}\right)+\frac{D_{z}^{\prime}}{D}\left(e^{-t}\right)\right] .
$$

By substituting the results from above in 227 and from 224 we obtain,

$$
\begin{aligned}
e^{t} & \left(Y^{-1} Y_{z}^{\prime}\right)_{22}\left(e^{t}\right)=-n+e^{t}\left(P^{-1} P_{z}^{\prime}\right)_{22}\left(e^{t}\right)+\left(\hat{\Phi}^{-1}(t) \mathcal{O}(1 / n) \hat{\Phi}(t)\right)_{22} \\
= & -n+\frac{\alpha_{0}+\beta_{0}}{4}+\frac{\alpha_{0}-\beta_{0}}{4}\left(\frac{1}{t}+\frac{e^{-t}}{\sinh t}\right)+\frac{1}{2} e^{t} V_{z}^{\prime}\left(e^{t}\right)-\frac{\alpha_{1}-\beta_{1}}{2}+\alpha_{1} \frac{e^{t}}{e^{t}-z_{1}}+e^{t}\left(\Phi^{-1} \Phi_{z}^{\prime}\right)_{11}\left(e^{t}\right) \\
& -\left\{\frac{\alpha_{0}+\beta_{0}}{4}+\frac{\alpha_{0}-\beta_{0}}{4}\left(\frac{1}{t}+\frac{e^{-t}}{\sinh t}\right)-\left(\alpha_{1}-\beta_{1}\right)\left(\frac{e^{t}+z_{1}}{2\left(e^{t}-z_{1}\right)}\right)+\alpha_{1} \frac{e^{t}}{e^{t}-z_{1}}+\frac{1}{2} e^{t} V_{z}^{\prime}\left(e^{t}\right)\right. \\
& \left.-\sum_{k=-\infty}^{-1} k V_{k} e^{t k}\right\}\left(\Phi^{-1} \sigma_{3} \Phi\right)_{11}\left(e^{t}\right)+\left(\hat{\Phi}^{-1}(t) \mathcal{O}(1 / n) \hat{\Phi}(t)\right)_{22}
\end{aligned}
$$

and

$$
\begin{aligned}
& e^{-t}\left(Y^{-1} Y_{z}^{\prime}\right)_{22}\left(e^{-t}\right)=e^{-t}\left(P^{-1} P_{z}^{\prime}\right)_{22}\left(e^{-t}\right)+\left(\hat{\Phi}^{-1}(t) \mathcal{O}(1 / n) \hat{\Phi}(t)\right)_{22} \\
&=-\frac{\alpha_{0}-\beta_{0}}{4}-\left(\frac{\alpha_{0}+\beta_{0}}{4}\right)\left(\frac{1}{t}+\frac{e^{-t}}{\sinh t}\right)+\frac{1}{2} e^{-t} V_{z}^{\prime}\left(e^{-t}\right)-\frac{\alpha_{1}-\beta_{1}}{2}+\alpha_{1} \frac{e^{-t}}{e^{-t}-z_{1}} \\
& \quad+e^{-t}\left(\Phi^{-1} \Phi_{z}^{\prime}\right)_{22}\left(e^{-t}\right)-\left\{\frac{\alpha_{0}-\beta_{0}}{4}+\frac{\alpha_{0}+\beta_{0}}{4}\left(\frac{1}{t}+\frac{e^{-t}}{\sinh t}\right)-\frac{\alpha_{1}-\beta_{1}}{2}+\beta_{1} \frac{e^{-t}}{e^{-t}-z_{1}}\right. \\
&\left.-\frac{1}{2} e^{-t} V_{z}^{\prime}\left(e^{-t}\right)+\sum_{k=0}^{\infty} k V_{k} e^{-t k}\right\}\left(\Phi^{-1} \sigma_{3} \Phi\right)\left(e^{-t}\right)+\left(\hat{\Phi}^{-1}(t) \mathcal{O}(1 / n) \hat{\Phi}(t)\right)_{22} .
\end{aligned}
$$

Therefore,

$$
\frac{d}{d t} \log D_{n}(t)=\left(\alpha_{0}+\beta_{0}\right) n-\frac{\alpha_{0}^{2}+\beta_{0}^{2}}{2}-\frac{\alpha_{0}^{2}-\beta_{0}^{2}}{2}\left(\frac{1}{t}+\frac{e^{-t}}{\sinh t}\right)+\beta_{0}\left(\alpha_{1}-\beta_{1}\right)
$$




$$
\begin{aligned}
& +\alpha_{1}\left[-\left(\alpha_{0}+\beta_{0}\right) \frac{e^{t}}{e^{t}-z_{1}}+\left(\alpha_{0}-\beta_{0}\right) \frac{e^{-t}}{e^{-t}-z_{1}}\right]-\frac{\alpha_{0}+\beta_{0}}{2} e^{t} V_{z}^{\prime}\left(e^{t}\right) \\
& +\frac{\alpha_{0}-\beta_{0}}{2} e^{-t} V_{z}^{\prime}\left(e^{-t}\right)+2 n w(x)+\left(\Phi^{-1} \sigma_{3} \Phi_{z}^{\prime}\right)_{11}\left(e^{t}\right) \times\left(\frac{\alpha_{0}+\beta_{0}}{2}\right) \\
& \times\left(\frac{\alpha_{0}+\beta_{0}}{2}+\frac{\alpha_{0}-\beta_{0}}{2}\left(\frac{1}{t}-\frac{e^{-t}}{\sinh t}\right)+\left(\alpha_{1}-\beta_{1}\right)+\frac{\beta_{1} e^{t}}{e^{t}-z_{1}}+\sum_{k=0}^{\infty} k V_{k} e^{t k}-\sum_{k=-\infty}^{-1} k V_{k} e^{t k}\right) \\
& -\left(\Phi^{-1} \sigma_{3} \Phi_{z}^{\prime}\right)_{22}\left(e^{-t}\right) \times\left(\frac{\alpha_{0}-\beta_{0}}{2}\right)\left(-\frac{\alpha_{0}-\beta_{0}}{2}-\frac{\alpha_{0}+\beta_{0}}{2}\left(\frac{1}{t}-\frac{e^{-t}}{\sinh t}\right)\right. \\
& \left.-\left(\alpha_{1}-\beta_{1}\right)+\frac{\beta_{1} e^{-t}}{e^{-t}-z_{1}}+\sum_{k=0}^{\infty} k V_{k} e^{-t k}-\sum_{k=-\infty}^{-1} k V_{k} e^{-t k}\right)+\hat{\Phi}^{-1} \mathcal{O}(1 / n) \hat{\Phi}(t)
\end{aligned}
$$

where

$$
w(x)=\frac{\alpha_{0}+\beta_{0}}{2}\left(\Phi^{-1} \Phi_{\lambda}^{\prime}\right)_{11}(x / 2)+\frac{\alpha_{0}-\beta_{0}}{2}\left(\Phi^{-1} \Phi_{\lambda}^{\prime}\right)(-x / 2) .
$$

We need the following two results, which are related to the Painlevé V function $\sigma$.

Proposition 10. [3, Proposition 4.4] Set

$$
a(\zeta ; x)=\left(\Psi(\zeta ; x) \sigma_{3} \Psi^{-1}(\zeta ; x)\right)_{11}=-\left(\Psi(\zeta ; x) \sigma_{3} \Psi^{-1}(\zeta ; x)\right)_{22}
$$

Then we have the following identities,

$$
\begin{gathered}
\frac{\alpha_{0}-\beta_{0}}{2} a(0 ; x)=A_{0,11}=-v(x)+\frac{\alpha_{0}-\beta_{0}}{2}, \\
\frac{\alpha_{0}+\beta_{0}}{2} a(1 ; x)=-A_{1,11}=-v(x)+\frac{\alpha_{0}+\beta_{0}}{2} .
\end{gathered}
$$

It is also worth nothing that $\left[\Psi(\zeta ; x) \sigma_{3} \Psi^{-1}(\zeta ; x)\right]_{\text {diag }}=\left[\Phi(\zeta ; x) \sigma_{3} \Phi^{-1}(\zeta ; x)\right]_{\text {diag }}$.

Proposition 11. [3, Proposition 4.5] If we have $w(x)$ given by 236 then,

$$
\begin{gathered}
v(x)=-(x w(x))^{\prime} \\
\sigma(x)=x w(x) \\
\sigma(x)=\int_{x}^{+\infty} v(\xi) d \xi
\end{gathered}
$$

Since $\left(\Phi(\zeta ; x) \sigma_{3} \Phi^{-1}\right)_{11}\left(e^{t}\right)=a(1 ; x)$ and $\left(\Phi(\zeta ; x) \sigma_{3} \Phi^{-1}\right)_{22}\left(e^{t}\right)=-a(0 ; x)$, we get

$$
\begin{aligned}
& \frac{d}{d t} \log D_{n}(t)=\left(\alpha_{0}+\beta_{0}\right) n-\left(\alpha_{0}^{2}-\beta_{0}^{2}\right) \frac{e^{-t}}{\sinh t}+\left(\alpha_{0}+\beta_{0}\right)\left(\alpha_{1}-\beta_{1}\right)-\left(\alpha_{1}-\beta_{1}\right)\left(\alpha_{0}+\beta_{0}\right) \frac{e^{t}}{e^{t}-z_{1}} \\
& \quad+\left(\alpha_{1}+\beta_{1}\right)\left(\alpha_{0}-\beta_{0}\right) \frac{e^{-t}}{e^{-t}-z_{1}}+\left(\alpha_{0}+\beta_{0}\right) \sum_{k=1}^{\infty} k V_{-k} e^{-t k}+\left(\alpha_{0}-\beta_{0}\right) \sum_{k=1}^{\infty} V_{k} e^{-t k}+\frac{1}{t} \sigma(x) \\
& \quad-v(x)\left\{\alpha_{0}+\alpha_{0}\left(\frac{1}{t}-\frac{e^{-t}}{\sinh t}\right)+2 \beta_{1}\left(\frac{e^{t}}{e^{t}-z_{1}}+\frac{e^{-t}}{e^{-t}-z_{1}}\right)+2 \sum_{k=1}^{\infty} k \cosh (k t)\left(V_{k}+V_{-k}\right)\right\} \\
& \quad+\hat{\Phi}^{-1} \mathcal{O}(1 / n) \hat{\Phi}(t) .
\end{aligned}
$$


When integrating this differential identity, we use the following integral and its two forms:

$$
\begin{gathered}
\int_{\varepsilon}^{t} \frac{e^{\tau}}{e^{\tau}-z_{1}} d \tau=\log \left(1-z_{1} e^{-t}\right)-\log \left(1-z_{1} e^{-\varepsilon}\right)+t-\varepsilon \\
\int_{\varepsilon}^{t} \frac{e^{-\tau}}{e^{-\tau}-z_{1}} d \tau=-\log \left(\frac{z_{1}}{e^{i \pi}}\right)-\log \left(1-z_{1}^{-1} e^{-t}\right)+\log \left(e^{-\varepsilon}-z_{1}\right) .
\end{gathered}
$$

We integrate the differential identity from $\varepsilon>0$ to some $t$, where $0<t<t_{0}$, and $0<\varepsilon<t$. Noting that $\int_{\varepsilon}^{t} \frac{d}{d t} \log D_{n}(\tau) d \tau=\log D_{n}(t)-\log D_{n}(\varepsilon)$, we obtain

$$
\begin{aligned}
& \log D_{n}(t)=\log D_{n}(\varepsilon)+\left(\alpha_{0}+\beta_{0}\right) n(t-\varepsilon)+\sum_{k=1}^{\infty} k\left[V_{k}-\left(\alpha_{0}+\beta_{0}\right) \frac{e^{-t k}}{k}\right]\left[V_{-k}-\left(\alpha_{0}-\beta_{0}\right) \frac{e^{-t k}}{k}\right] \\
& \quad-\sum_{k=1}^{\infty} k V_{k} V_{-k}+\left(\alpha_{0}+\beta_{0}\right) \sum_{k=1}^{\infty} V_{-k} e^{-\varepsilon k}+\left(\alpha_{0}-\beta_{0}\right) \sum_{k=1}^{\infty} V_{k} e^{-\varepsilon k} \\
& \quad+\left(\alpha_{0}+\beta_{0}-\alpha_{0}-\beta_{0}\right)\left(\alpha_{1}-\beta_{1}\right)(t-\varepsilon)-\left(\alpha_{1}+\beta_{1}\right)\left(\alpha_{0}-\beta_{0}\right) \log \left(\frac{z_{1}}{e^{i \pi}}\right) \\
& \quad+\left(\alpha_{1}-\beta_{1}\right)\left(\alpha_{0}+\beta_{0}\right)\left(\sum_{k=1}^{\infty} \frac{z_{1}^{k} e^{-t k}}{k}\right)+\left(\alpha_{1}+\beta_{1}\right)\left(\alpha_{0}-\beta_{0}\right)\left(\sum_{k=1}^{\infty} \frac{z_{1}^{-k} e^{-t k}}{k}\right) \\
& \quad+\left(\alpha_{1}-\beta_{1}\right)\left(\alpha_{0}+\beta_{0}\right) \log \left(1-z_{1} e^{-\varepsilon}\right)+\left(\alpha_{1}+\beta_{1}\right)\left(\alpha_{0}-\beta_{0}\right) \log \left(e^{-\varepsilon}-z_{1}\right) \\
& +\left[\int_{2 n \varepsilon}^{2 n t} \frac{\sigma(x)-\left(\alpha_{0}^{2}-\beta_{0}^{2}\right)}{x} d x+\left(\alpha_{0}^{2}-\beta_{0}^{2}\right) \log (2 n t)+\left(\alpha_{0}^{2}-\beta_{0}^{2}\right) \log \left(\frac{n\left(1-e^{-2 \varepsilon}\right)}{2 n \epsilon}\right)\right] \\
& \quad-\left(\alpha_{0}^{2}-\beta_{0}^{2}\right) \log n+R_{n}(t)+\mathcal{O}(1 / n),
\end{aligned}
$$

where

$$
\begin{gathered}
R_{n}(t)=-\int_{\varepsilon}^{t} v(2 n t)\left\{\alpha_{0}+\alpha_{0}\left(\frac{1}{t}-\frac{e^{-t}}{\sinh t}\right)+2 \beta_{1}\left(\frac{e^{t}}{e^{t}-z_{1}}+\frac{e^{-t}}{e^{-t}-z_{1}}\right)\right. \\
\left.+2 \sum_{k=1}^{\infty} k \cosh (k t)\left(V_{k}+V_{-k}\right)\right\}
\end{gathered}
$$

Also, as in [3, (5.3)], we have

$$
\left|R_{n}(t)\right|<C \int_{0}^{t}|v(2 n u)| d u=\mathcal{O}(1 / n), \quad \text { as } n \rightarrow \infty, 0<t<t_{0}
$$

using

$$
-\left(\alpha_{0}^{2}-\beta_{0}^{2}\right) \log \left(1-e^{-2 t}\right)=\left(\alpha_{0}^{2}+\beta_{0}^{2}\right) \sum_{k=1}^{\infty} \frac{e^{-2 t k}}{k}=\sum_{k=1}^{\infty} k\left[\left(\alpha_{0}-\beta_{0}\right) \frac{e^{-t k}}{k}\right]\left[\left(\alpha_{0}+\beta_{0}\right) \frac{e^{-t k}}{k}\right] .
$$

Taking the limit $\varepsilon \rightarrow 0$, we get

$$
\begin{aligned}
& \log D_{n}(t)=n V_{0}+\sum_{k=1}^{\infty} k V_{k} V_{-k}-\left(\alpha_{0}-\beta_{0}\right) \sum_{k=1}^{\infty} V_{k}-\left(\alpha_{0}+\beta_{0}\right) \sum_{k=1}^{\infty} V_{-k}-\left(\alpha_{1}-\beta_{1}\right) \sum_{k=1}^{\infty} V_{k} z_{1}^{-k} \\
& \quad+\left(\alpha_{0}^{2}-\beta_{0}^{2}\right) \log n+\left(\alpha_{1}^{2}-\beta_{1}^{2}\right) \log n+2\left(\beta_{0} \beta_{1}-\alpha_{0} \alpha_{1}\right) \log \left(1-z_{1}\right) \\
& \quad+\left(\alpha_{0} \alpha_{1}-\beta_{0} \beta_{1}\right)\left(\log z_{1}+\log e^{i \pi}\right)+\left(\alpha_{0} \beta_{1}-\alpha_{1} \beta_{0}\right) \log \left(\frac{z_{1}}{e^{i \pi}}\right)+\log G_{\alpha_{0}, \beta_{0}} \\
& \quad+\log G_{\alpha_{1}, \beta_{1}}+\left(\alpha_{0}+\beta_{0}\right) n t+\sum_{k=1}^{\infty} k\left[V_{k}-\left(\alpha_{0}+\beta_{0}\right) \frac{e^{-t k}}{k}\right]\left[V_{-k}-\left(\alpha_{0}-\beta_{0}\right) \frac{e^{-t k}}{k}\right]
\end{aligned}
$$




$$
\begin{aligned}
& -\sum_{k=1}^{\infty} k V_{k} V_{-k}+\left(\alpha_{0}+\beta_{0}\right) \sum_{k=1}^{\infty} V_{-k}+\left(\alpha_{0}-\beta_{0}\right) \sum_{k=1}^{\infty} V_{k}-\left(\alpha_{1}+\beta_{1}\right)\left(\alpha_{0}-\beta_{0}\right) \log \left(\frac{z_{1}}{e^{i \pi}}\right) \\
& +\left(\alpha_{1}-\beta_{1}\right)\left(\alpha_{0}+\beta_{0}\right)\left(\sum_{k=1}^{\infty} \frac{z_{1}^{k} e^{-t k}}{k}\right)+\left(\alpha_{1}+\beta_{1}\right)\left(\alpha_{0}-\beta_{0}\right)\left(\sum_{k=1}^{\infty} \frac{z_{1}^{-k} e^{-t k}}{k}\right) \\
& +\left(\alpha_{1}-\beta_{1}\right)\left(\alpha_{0}+\beta_{0}\right) \log \left(1-z_{1}\right)+\left(\alpha_{1}+\beta_{1}\right)\left(\alpha_{0}-\beta_{0}\right) \log \left(1-z_{1}\right) \\
& +\left[\int_{0}^{2 n t} \frac{\sigma(x)-\left(\alpha_{0}^{2}-\beta_{0}^{2}\right)}{x} d x+\left(\alpha_{0}^{2}-\beta_{0}^{2}\right) \log (2 n t)\right]-\left(\alpha_{0}^{2}-\beta_{0}^{2}\right) \log n+\mathcal{O}(1 / n) .
\end{aligned}
$$

After cancellations, we obtain the expression

$$
\begin{aligned}
& \log D_{n}\left(f_{t}\right)=n V_{0}+n t\left(\alpha_{0}+\beta_{0}\right)+\sum_{k=1}^{\infty} k\left[V_{k}-\left(\alpha_{0}+\beta_{0}\right) \frac{e^{-t k}}{k}\right]\left[V_{-k}-\left(\alpha_{0}-\beta_{0}\right) \frac{e^{-t k}}{k}\right] \\
& -\left(\alpha_{1}-\beta_{1}\right) \sum_{k=1}^{\infty}\left[\left(V_{k}-\left(\alpha_{0}+\beta_{0}\right) \frac{e^{-t k}}{k}\right) z_{1}^{k}\right]-\left(\alpha_{1}+\beta_{1}\right) \sum_{k=1}^{\infty}\left[\left(V_{-k}-\left(\alpha_{0}-\beta_{0}\right) \frac{e^{-t k}}{k}\right) z_{1}^{-k}\right] \\
& +\left(\alpha_{1}^{2}-\beta_{1}^{2}\right) \log n+\log G_{\alpha_{0}, \beta_{0}}+\log G_{\alpha_{1}, \beta_{1}} \\
& +\left[\int_{0}^{2 n t} \frac{\sigma(x)-\left(\alpha_{0}^{2}-\beta_{0}^{2}\right)}{x} d x+\left(\alpha_{0}^{2}-\beta_{0}^{2}\right) \log (2 n t)\right]+\mathcal{O}(1 / n) .
\end{aligned}
$$

Remark 12. The general case of $\alpha_{0}, \beta_{0} \in \mathbb{C}$ can be dealt with using the same arguments as in [3, Theorem 1.4]. Indeed, let $\operatorname{Re} \alpha_{0}>-1 / 2, \alpha_{0} \pm \beta_{0} \neq-1,-2, \ldots$, and let $s_{\delta}$ denote a sector $-\pi / 2+\delta<\arg x<\pi / 2-\delta, 0<\delta<\pi / 2$. Let $f_{t}$ be the symbol in (38). There is a finite set $\left\{x_{1}, \ldots, x_{l}\right\} \in s_{\delta}$ (with $l=l\left(\alpha_{0}, \beta_{0}, \delta\right)$ and $x_{j}=x_{j}\left(\alpha_{0}, \beta_{0}\right) \neq 0$ ) such that the expansion (43) holds uniformly for $t \in s_{\delta},|t|<t_{0}$ (with $t_{0}$ sufficiently small) as long as $2 n t$ remains bounded away from the set $\left\{x_{1}, \ldots, x_{l}\right\}$. The function $\widetilde{\Omega}$ is defined by (44), where the path of integration is chosen in $s_{\delta}$, connecting 0 with $2 n t$ and not containing any of the points $x_{1}, \ldots, x_{l}$. Moreover $\sigma$ solves the ODE mentioned in Theorem 5 and has the asymptotics in $s_{\delta}$ given by (45).

\section{Proof of Theorem 6}

In this section we will make use of a lemma and the approach that was presented in [6] to prove the Tracy-Basor conjecture (Theorem 4). We only give the particular case of this lemma here.

In this section we consider the case when the seminorm (see (8D) is equal to 1 . This means that we can write $\operatorname{Re} \beta_{0}=q-1 / 2$ and $\operatorname{Re} \beta_{1}=q+1 / 2$ for some $q \in \mathbb{R}$. Without loss of generality we may assume that $\Re \beta_{0}<\Re \beta_{1}$. Note that the symbol (38) has only one F-H singularity for $t>0$, but two $\beta$ parameters. If we translate these parameters, we will not get a $\mathrm{F}-\mathrm{H}$ representation as it was presented in Definition 2. The symbol we obtain by shifting $\beta_{j}$ will vary by more than just a multiplicative constant; that is,

$$
\begin{aligned}
f(z ; t)= & e^{V(z)} z^{\beta_{1}}\left|z-z_{1}\right|^{2 \alpha_{1}} g_{z_{1}, \beta_{1}}(z) z_{1}^{-\beta_{1}}\left(z-e^{t}\right)^{\alpha_{0}+\beta_{0}}\left(z-e^{-t}\right)^{\alpha_{0}-\beta_{0}} z^{-\alpha_{0}+\beta_{0}} e^{-i \pi\left(\alpha_{0}+\beta_{0}\right)} \\
= & e^{V(z)} z^{\left(\beta_{1}-1\right)+1}\left|z-z_{1}\right|^{2 \alpha_{1}} g_{z_{1},\left(\beta_{1}-1\right)+1}(z) z_{1}^{-\left(\beta_{1}-1\right)-1} \\
& \quad \times\left(z-e^{t}\right)^{\alpha_{0}+\left(\beta_{0}+1\right)-1}\left(z-e^{-t}\right)^{\alpha_{0}-\left(\beta_{0}+1\right)+1} z^{-\alpha_{0}+\left(\beta_{0}+1\right)-1} e^{-i \pi\left(\alpha_{0}+\left(\beta_{0}+1\right)-1\right)} \\
= & \frac{\left(z-e^{-t}\right)}{\left(z-e^{t}\right)} z_{1}^{-1} \tilde{f}(z ; t),
\end{aligned}
$$

where $\tilde{f}(z ; t)$ is $(38)$ with $\beta_{0}$ and $\beta_{1}$ replaced by $\tilde{\beta}_{0}=\beta_{0}+1$ and $\tilde{\beta}_{1}=\beta_{1}-1$, respectively. Notice that shifting $\beta_{1}$, which is associated with the F-H singularity still produces the multiplicative constant 
we know. Following the idea of the proof in [6, Section 6$]$, we define a new symbol $\hat{f}(z ; t)$ which is given by $(38)$ but whose $\beta$-parameters are replaced by $\hat{\beta}_{j}$ where $\hat{\beta}_{0}=\beta_{0}$ and $\hat{\beta}_{1}=\beta_{1}-1$. For this new symbol $\hat{f}(z ; t)$, we have $\|\beta\| \mid<1$ and we have computed the asymptotics of the corresponding Toeplitz matrices, which are given by 248 — with the $\beta_{j}$ parameters replaced by $\hat{\beta}_{j}$. We will simply try to relate the two symbols, the original symbol $f(z ; t)$ with $\|\beta\|=1$ and $\hat{f}(z ; t)$, and make use of the asymptotics we already know for $\hat{f}(z ; t)$ in order to compute the asymptotics for $f(z ; t)$. We can obtain the original symbol by shifting $\hat{\beta}_{1}$ in $\hat{f}(z ; t)$ back by +1 , which is what is called a trivial $\mathrm{F}-\mathrm{H}$ representation. Alternatively, we can shift $\beta_{0}$ by +1 to obtain a representation corresponding to $\tilde{\beta}_{j}$ from 249 above. Thus,

$$
f(z ; t)=(-1) z_{1}^{-1} z \hat{f}(z ; t)
$$

and

$$
\tilde{f}(z ; t)=(-1) \frac{z\left(z-e^{t}\right)}{\left(z-e^{-t}\right)} \hat{f}(z ; t) .
$$

It is sufficient to consider only one of the above relations. We pick 250 and make use of the following lemma.

Lemma 13. 6. Lemma 2.4] Let the Toeplitz determinants $D_{n}(f)$ with symbol $f(z)$ be non-zero for all $n \geq N_{0}$ with a fixed $N_{0} \geq 0$. If $\phi_{k}(0) \neq 0, k=N_{0}, N_{0}+1, \ldots, n-1$, we have

$$
D_{n}(z f(z))=(-1)^{n} \frac{\phi_{n}(0)}{\chi_{n}} D_{n}(f(z)), \quad n \geq N_{0},
$$

where $\chi_{n}$ is the leading coefficient of the polynomial $\phi(z)$, see 50 .

The proof uses Christoffel's formula [26, Theorem 2.5] to represent new orthogonal polynomials, say $q_{n}(z)$, orthogonal with respect to some weight $\rho(z) f(z)$ (where $\rho(z)$ is a polynomial), in terms of polynomials $\phi_{n}(z)$, which are orthogonal with respect to the weight $f(z)$. Using orthogonality conditions (50) and relating the leading coefficients $\chi_{n}$ via (54) one can link the Toeplitz determinants with the weights that vary by a polynomial $\rho(z)$.

Thus, by (250) and (252), using the uniform asymptotics in Section 8 and asymptotics of the polynomials orthogonal with respect to $\hat{f}(z ; t)$, we get

$$
D_{n}(f)=z_{1}^{-n} \frac{\phi_{n}(0)}{\chi_{n}} D_{n}(\hat{f})
$$

\subsection{Asymptotics for the Orthogonal Polynomials.}

Lemma 14. Let $t>0$ and $n \in \mathbb{N}$. Suppose that the $R-H$ problem for $Y(z ; n, t)$ in Section 5 is solvable with $\hat{f}$ given by (38) with $\beta$-parameters replaced by $\hat{\beta},\|\hat{\beta}\| \mid<1$ and $\alpha_{j} \pm \hat{\beta}_{j} \neq-1,-2, \ldots$, $j=0,1$. Let $\phi$ and $\hat{\phi}$ be the orthogonal polynomials associated to the weight $\hat{f}(z)$. Then as $n \rightarrow \infty$,

$$
\begin{aligned}
\frac{\phi_{n}(0)}{\chi_{n}}= & {\left[z_{1}^{n}\left(1-z_{1} e^{-t}\right)^{\left(\alpha_{0}+\hat{\beta}_{0}\right)}\left(1-e^{-t} z_{1}^{-1}\right)^{-\left(\alpha_{0}-\hat{\beta}_{0}\right)} \frac{\exp \left\{\sum_{k=1}^{\infty} V_{k} z_{1}^{k}\right\}}{\exp \left\{\sum_{k=1}^{\infty} V_{-k} z_{k}^{-k}\right\}}\right.} \\
& \times n^{-2 \hat{\beta}_{1}-1} \frac{\Gamma\left(1+\alpha_{1}+\hat{\beta}_{1}\right)}{\Gamma\left(\alpha_{1}-\hat{\beta}_{1}\right)} \\
+ & \left(1-z_{1} e^{-t}\right)^{2 \hat{\beta}_{1}}\left(1-e^{-2 t}\right)^{-\left(\alpha_{0}-\hat{\beta}_{0}\right)} \frac{\exp \left\{\sum_{k=1}^{\infty} V_{k} e^{t k}\right\}}{\exp \left\{\sum_{k=1}^{\infty} V_{-k} e^{-t k}\right\}} e^{t\left(\alpha_{1}+\hat{\beta}_{1}\right)}
\end{aligned}
$$




$$
\begin{aligned}
& \times e^{-i \pi\left(\alpha_{1}+\hat{\beta}_{1}\right)} z_{1}^{-\left(\alpha_{1}+\hat{\beta}_{1}\right)}(2 t)^{\alpha_{0}-\hat{\beta}_{0}} \frac{n^{-2 \hat{\beta}_{0}-1}}{\Gamma\left(\alpha_{0}-\hat{\beta}_{0}\right)} K(2 n t) \\
& +\left(1-z_{1}^{-1} e^{-t}\right)^{2 \hat{\beta}_{1}}\left(1-e^{-2 t}\right)^{\alpha_{0}+\hat{\beta}_{0}} \frac{\exp \left\{\sum_{k=1}^{\infty} V_{k} e^{-t k}\right\}}{\exp \left\{\sum_{k=1}^{\infty} V_{-k} e^{t k}\right\}} e^{-t\left(\alpha_{1}-\hat{\beta}_{1}\right)} \\
& \left.\quad \times e^{-i \pi\left(\alpha_{1}-\hat{\beta}_{1}\right)} z_{1}^{-\left(\alpha_{1}-\hat{\beta}_{1}\right)}(2 t)^{-\left(\alpha_{0}+\hat{\beta}_{0}\right)} \frac{n^{-2 \hat{\beta}_{0}-1}}{\Gamma\left(\alpha_{0}-\hat{\beta}_{0}\right)} K(2 n t)\right](1+o(1)) .
\end{aligned}
$$

Proof. We find the asymptotics for the orthogonal polynomials in (253). Recall (55) and $Y_{11}(0)=$ $\phi_{n}(0) / \chi_{n}$. Now, going through the transformations $R \mapsto S \mapsto T \mapsto Y$, and using the Neumann series solution to $R$, we obtain

$$
Y(z)=\left[I+R_{1}(z)+R_{2}(z)+R_{3}^{(r)}(z)\right] D(z)^{\sigma_{3}}\left(\begin{array}{cc}
0 & 1 \\
-1 & 0
\end{array}\right)
$$

which in turn leads to,

$$
\frac{\phi_{n}(0)}{\chi_{n}}=Y_{11}(0)=-D(0)^{-1}\left[R_{1,12}(0)+R_{2,12}(0)+\mathcal{O}\left(\delta \max _{k} \frac{n^{-2 \Re \beta_{k}}}{n}\right)\right],
$$

where we used (220) and $\delta$ is given by 221). By 279 or (81), $D(0)=e^{V_{0}} e^{t\left(\alpha_{0}+\hat{\beta}_{0}\right)}$, and by (213),

$$
R_{1}(0)=-\frac{A_{1}}{z_{1}}-\frac{A_{e^{t}}}{e^{t}}-\frac{A_{e^{-t}}}{e^{-t}} .
$$

Finally, it follows from (214), 215), 216, 217), and (218) that

$$
\begin{aligned}
& R_{1,12}(0)= e^{V_{0}} e^{t\left(\alpha_{0}+\hat{\beta}_{0}\right)}\left[-\frac{1}{n} z_{1}^{n}\left(1-z_{1} e^{-t}\right)^{\left(\alpha_{0}+\hat{\beta}_{0}\right)}\left(1-e^{-t} z_{1}^{-1}\right)^{-\left(\alpha_{0}-\hat{\beta}_{0}\right)}\right. \\
& \times \frac{\exp \left\{\sum_{k=1}^{\infty} V_{k} z_{1}^{k}\right\}}{\exp \left\{-\sum_{k=1}^{\infty} V_{-k} z_{k}^{-k}\right\}} n^{-2 \hat{\beta}_{1}} \frac{\Gamma\left(1+\alpha_{1}+\hat{\beta}_{1}\right)}{\Gamma\left(\alpha_{1}-\hat{\beta}_{1}\right)}(1+o(1)) \\
&-\frac{1}{n}\left(1-z_{1} e^{-t}\right)^{2 \hat{\beta}_{1}}\left(1-e^{-2 t}\right)^{-\left(\alpha_{0}-\hat{\beta}_{0}\right)} \frac{\exp \left\{\sum_{k=1}^{\infty} V_{k} e^{t k}\right\}}{\exp \left\{-\sum_{k=1}^{\infty} V_{-k} e^{-t k}\right\}} e^{t\left(\alpha_{1}+\hat{\beta}_{1}\right)} \\
& \times e^{-i \pi\left(\alpha_{1}+\hat{\beta}_{1}\right)} z_{1}^{-\left(\alpha_{1}+\hat{\beta}_{1}\right)}(2 t)^{\alpha_{0}-\hat{\beta}_{0}} \frac{n^{-2 \hat{\beta}_{0}}}{\Gamma\left(\alpha_{0}-\hat{\beta}_{0}\right)} K(2 n t)(1+o(1)) \\
&-\frac{1}{n}\left(1-z_{1}^{-1} e^{-t}\right)^{2 \hat{\beta}_{1}}\left(1-e^{-2 t}\right)^{\alpha_{0}+\hat{\beta}_{0}} \frac{\exp \left\{\sum_{k=1}^{\infty} V_{k} e^{-t k}\right\}}{\exp \left\{-\sum_{k=1}^{\infty} V_{-k} e^{t k}\right\}} e^{-t\left(\alpha_{1}-\hat{\beta}_{1}\right)} \\
&\left.\quad \times e^{-i \pi\left(\alpha_{1}-\hat{\beta}_{1}\right)} z_{1}^{-\left(\alpha_{1}-\hat{\beta}_{1}\right)}(2 t)^{-\left(\alpha_{0}+\hat{\beta}_{0}\right)} \frac{n^{-2 \hat{\beta}_{0}}}{\Gamma\left(\alpha_{0}-\hat{\beta}_{0}\right)} K(2 n t)(1+o(1))\right] .
\end{aligned}
$$

We thus obtain the asymptotics in (254).

9.2. Asymptotics for the determinant. We use the relation between two Toeplitz determinants we established in (253). By the properties of the Barnes $G$-function, $G(z+1)=\Gamma(z) G(z)$ and (11),

$$
G_{\alpha_{j}+\beta_{j}+1, \alpha_{j}-\beta_{j}-1}=\frac{G\left(1+\alpha_{j}+\beta_{j}+1\right) G\left(1+\alpha_{j}-\beta_{j}-1\right)}{G\left(1+2 \alpha_{j}\right)}=\frac{\Gamma\left(1+\alpha_{j}+\beta_{j}\right)}{\Gamma\left(\alpha_{j}-\beta_{j}\right)} G_{\alpha_{j}+\beta_{j}, \alpha_{j}-\beta_{j}}
$$

where

$$
G_{x, y}=\frac{G(1+x) G(1+y)}{G(1+x+y)}
$$


Write

$$
\tilde{\Omega}(2 n t)=\exp \{\Omega(2 n t)\}=\exp \left\{\int_{0}^{2 n t} \frac{\sigma(x)-\left(\alpha_{0}^{2}-\beta_{0}^{2}\right)}{x} d x+\left(\alpha_{0}^{2}-\beta_{0}^{2}\right) \log (2 n t)\right\},
$$

(see (44) and (248)) and note that there is no shift in $\beta_{0}=\hat{\beta_{0}}$. By 253) and 254),

$$
\begin{aligned}
& D_{n}(f)=\exp \left\{n V_{0}+n t\left(\alpha_{0}+\hat{\beta}_{0}\right)\right\} \exp \left\{\sum_{k=1}^{\infty} k\left[V_{k}-\left(\alpha_{0}+\hat{\beta}_{0}\right) \frac{e^{-t k}}{k}\right]\left[V_{-k}-\left(\alpha_{0}-\hat{\beta}_{0}\right) \frac{e^{-t k}}{k}\right]\right\} \\
& \times \exp \left\{-\left(\alpha_{1}-\hat{\beta}_{1}-1\right) \sum_{k=1}^{\infty}\left[\left(V_{k}-\left(\alpha_{0}+\hat{\beta}_{0}\right) \frac{e^{-t k}}{k}\right) z_{1}^{k}\right]\right\} \\
& \times \exp \left\{-\left(\alpha_{1}+\hat{\beta}_{1}+1\right) \sum_{k=1}^{\infty}\left[\left(V_{-k}-\left(\alpha_{0}-\hat{\beta}_{0}\right) \frac{e^{-t k}}{k}\right) z_{1}^{-k}\right]\right\} n^{\left(\alpha_{1}^{2}-\hat{\beta}_{1}^{2}-2 \hat{\beta}_{1}-1\right)} \\
& \times G_{\alpha_{0}, \hat{\beta}_{0}} G_{\alpha_{1}, \hat{\beta}_{1}+1} \tilde{\Omega}(2 n t)(1+o(1)) \\
& +\exp \left\{n V_{0}+n t\left(\alpha_{0}+\hat{\beta}_{0}\right)\right\} \exp \left\{\sum_{k=1}^{\infty} k\left[V_{k}-\left(\alpha_{0}+\hat{\beta}_{0}+1\right) \frac{e^{-t k}}{k}\right]\left[V_{-k}-\left(\alpha_{0}-\hat{\beta}_{0}\right) \frac{e^{-t k}}{k}\right]\right\} \\
& \times \exp \left\{-\left(\alpha_{1}-\hat{\beta}_{1}\right) \sum_{k=1}^{\infty}\left[\left(V_{k}-\left(\alpha_{0}+\hat{\beta}_{0}+1\right) \frac{e^{-t k}}{k}\right) z_{1}^{k}\right]\right\}\left(1-e^{t} z_{1}^{-1}\right)^{\alpha_{1}+\hat{\beta}_{1}} \exp \left\{\sum_{k=1}^{\infty} V_{k} e^{t k}\right\} \\
& \times \exp \left\{-\left(\alpha_{1}+\hat{\beta}_{1}\right) \sum_{k=1}^{\infty}\left[\left(V_{-k}-\left(\alpha_{0}-\hat{\beta}_{0}\right) \frac{e^{-t k}}{k}\right) z_{1}^{-k}\right]\right\} n^{\left(\alpha_{1}^{2}-\hat{\beta}_{1}^{2}\right)} n^{-2 \beta_{0}-1} \\
& \times z_{1}^{-n}(2 t)^{\alpha_{0}-\beta_{0}} \frac{G_{\alpha_{0}, \hat{\beta}_{0}+1}}{\Gamma\left(1+\alpha_{0}+\widehat{\beta}_{0}\right)} G_{\alpha_{1}, \hat{\beta}_{1}} \tilde{\Omega}(2 n t) K(2 n t)(1+o(1)) \\
& +\exp \left\{n V_{0}+n t\left(\alpha_{0}+\hat{\beta}_{0}\right)\right\} \exp \left\{\sum_{k=1}^{\infty} k\left[V_{k}-\left(\alpha_{0}+\hat{\beta}_{0}\right) \frac{e^{-t k}}{k}\right]\left[V_{-k}-\left(\alpha_{0}-\hat{\beta}_{0}-1\right) \frac{e^{-t k}}{k}\right]\right\} \\
& \times \exp \left\{-\left(\alpha_{1}-\hat{\beta}_{1}\right) \sum_{k=1}^{\infty}\left[\left(V_{k}-\left(\alpha_{0}+\hat{\beta}_{0}\right) \frac{e^{-t k}}{k}\right) z_{1}^{k}\right]\right\}\left(1-e^{t} z_{1}\right)^{-\left(\alpha_{1}-\hat{\beta}_{1}\right)} \exp \left\{-\sum_{k=1}^{\infty} V_{-k} e^{t k}\right\} \\
& \times \exp \left\{-\left(\alpha_{1}+\hat{\beta}_{1}\right) \sum_{k=1}^{\infty}\left[\left(V_{-k}-\left(\alpha_{0}-\hat{\beta}_{0}-1\right) \frac{e^{-t k}}{k}\right) z_{1}^{-k}\right]\right\} n^{\left(\alpha_{1}^{2}-\hat{\beta}_{1}^{2}\right)} n^{-2 \beta_{0}-1}
\end{aligned}
$$

$$
\times z_{1}^{-n}(2 t)^{-\left(\alpha_{0}+\beta_{0}\right)} \frac{G_{\alpha_{0}, \hat{\beta}_{0}+1}}{\Gamma\left(1+\alpha_{0}+\widehat{\beta}_{0}\right)} G_{\alpha_{1}, \hat{\beta}_{1}} \tilde{\Omega}(2 n t) K(2 n t)(1+o(1)) .
$$

Recalling that $\hat{\beta}_{0}=\beta_{0}$ and $\hat{\beta}_{1}=\beta_{1}-1$, and using $\tilde{\beta}_{0}=\beta_{0}+1, \tilde{\beta}_{1}=\beta_{1}-1($ see $(249)$ ),

$$
\begin{aligned}
& D_{n}(f)=\exp \left\{n V_{0}+n t\left(\alpha_{0}+\beta_{0}\right)\right\} \exp \left\{\sum_{k=1}^{\infty} k\left[V_{k}-\left(\alpha_{0}+\beta_{0}\right) \frac{e^{-t k}}{k}\right]\left[V_{-k}-\left(\alpha_{0}-\beta_{0}\right) \frac{e^{-t k}}{k}\right]\right\} \\
& \quad \times \exp \left\{-\left(\alpha_{1}-\beta_{1}\right) \sum_{k=1}^{\infty}\left[\left(V_{k}-\left(\alpha_{0}+\beta_{0}\right) \frac{e^{-t k}}{k}\right) z_{1}^{k}\right]\right\}
\end{aligned}
$$




$$
\begin{aligned}
& \times \exp \left\{-\left(\alpha_{1}+\beta_{1}\right) \sum_{k=1}^{\infty}\left[\left(V_{-k}-\left(\alpha_{0}-\beta_{0}\right) \frac{e^{-t k}}{k}\right) z_{1}^{-k}\right]\right\} n^{\left(\alpha_{1}^{2}-\beta_{1}^{2}\right)} \\
& \times G_{\alpha_{0}, \beta_{0}} G_{\alpha_{1}, \beta_{1}} \tilde{\Omega}(2 n t)(1+o(1)) \\
& +\exp \left\{n V_{0}+n t\left(\alpha_{0}+\beta_{0}\right)\right\} \exp \left\{\sum_{k=1}^{\infty} k\left[V_{k}-\left(\alpha_{0}+\tilde{\beta}_{0}\right) \frac{e^{-t k}}{k}\right]\left[V_{-k}-\left(\alpha_{0}-\beta_{0}\right) \frac{e^{-t k}}{k}\right]\right\} \\
& \times \exp \left\{-\left(\alpha_{1}-\tilde{\beta}_{1}\right) \sum_{k=1}^{\infty}\left[\left(V_{k}-\left(\alpha_{0}+\tilde{\beta}_{0}\right) \frac{e^{-t k}}{k}\right) z_{1}^{k}\right]\right\}\left(1-e^{t} z_{1}^{-1}\right)^{\alpha_{1}+\tilde{\beta}_{1}} \exp \left\{\sum_{k=1}^{\infty} V_{k} e^{t k}\right\} \\
& \times \exp \left\{-\left(\alpha_{1}+\tilde{\beta}_{1}\right) \sum_{k=1}^{\infty}\left[\left(V_{-k}-\left(\alpha_{0}-\beta_{0}\right) \frac{e^{-t k}}{k}\right) z_{1}^{-k}\right]\right\} n^{\left(\alpha_{1}^{2}-\tilde{\beta}_{1}^{2}\right)} n^{-2 \beta_{0}-1} \\
& \times z_{1}^{-n}(2 t)^{\alpha_{0}-\beta_{0}} \frac{G_{\alpha_{0}, \tilde{\beta}_{0}}}{\Gamma\left(1+\alpha_{0}+\beta_{0}\right)} G_{\alpha_{1}, \tilde{\beta}_{1}} \tilde{\Omega}(2 n t) K(2 n t)(1+o(1)) \\
& +\exp \left\{n V_{0}+n t\left(\alpha_{0}+\beta_{0}\right)\right\} \exp \left\{\sum_{k=1}^{\infty} k\left[V_{k}-\left(\alpha_{0}+\beta_{0}\right) \frac{e^{-t k}}{k}\right]\left[V_{-k}-\left(\alpha_{0}-\tilde{\beta}_{0}\right) \frac{e^{-t k}}{k}\right]\right\} \\
& \times \exp \left\{-\left(\alpha_{1}-\tilde{\beta}_{1}\right) \sum_{k=1}^{\infty}\left[\left(V_{k}-\left(\alpha_{0}+\beta_{0}\right) \frac{e^{-t k}}{k}\right) z_{1}^{k}\right]\right\}\left(1-e^{t} z_{1}\right)^{-\left(\alpha_{1}-\tilde{\beta}_{1}\right)} \exp \left\{-\sum_{k=1}^{\infty} V_{-k} e^{t k}\right\} \\
& \times \exp \left\{-\left(\alpha_{1}+\tilde{\beta}_{1}\right) \sum_{k=1}^{\infty}\left[\left(V_{-k}-\left(\alpha_{0}-\tilde{\beta}_{0}\right) \frac{e^{-t k}}{k}\right) z_{1}^{-k}\right]\right\} n^{\left(\alpha_{1}^{2}-\tilde{\beta}_{1}^{2}\right)} n^{-2 \beta_{0}-1} \\
& \times z_{1}^{-n}(2 t)^{-\left(\alpha_{0}+\beta_{0}\right)} \frac{G_{\alpha_{0}, \tilde{\beta}_{0}}}{\Gamma\left(1+\alpha_{0}+\beta_{0}\right)} G_{\alpha_{1}, \tilde{\beta}_{1}} \tilde{\Omega}(2 n t) K(2 n t)(1+o(1)) .
\end{aligned}
$$

By manipulating the above, we arrive at a more compact expression resulting in (47) of Theorem 6 .

Acknowledgments. The authors thank Tom Claeys, Percy Deift and Igor Krasovsky for useful discussions.

\section{REFERENCES}

[1] E. L. Basor and C. A. Tracy. The Fisher-Hartwig conjecture and generalizations. Phys. A, 177(1-3):167-173, 1991. Current problems in statistical mechanics (Washington, DC, 1991).

[2] A. Böttcher and B. Silbermann. Analysis of Toeplitz operators. Springer Monographs in Mathematics. SpringerVerlag, Berlin, second edition, 2006. Prepared jointly with Alexei Karlovich.

[3] T. Claeys, A. Its, and I. Krasovsky. Emergence of a singularity for Toeplitz determinants and Painlevé V. Duke Math. J., 160(2):207-262, 2011.

[4] T. Claeys and I. Krasovsky. Toeplitz determinants with merging singularities. Duke Math. J., 164(15):2897-2987, 2015.

[5] P. Deift. Integrable operators. In Differential operators and spectral theory, volume 189 of Amer. Math. Soc. Transl. Ser. 2, pages 69-84. Amer. Math. Soc., Providence, RI, 1999.

[6] P. Deift, A. Its, and I. Krasovsky. Asymptotics of Toeplitz, Hankel, and Toeplitz+Hankel determinants with Fisher-Hartwig singularities. Ann. of Math. (2), 174(2):1243-1299, 2011.

[7] P. Deift, A. Its, and I. Krasovsky. Toeplitz matrices and Toeplitz determinants under the impetus of the Ising model: some history and some recent results. Comm. Pure Appl. Math., 66(9):1360-1438, 2013.

[8] P. Deift, A. Its, and I. Krasovsky. On the asymptotics of a Toeplitz determinant with singularities. In Random matrix theory, interacting particle systems, and integrable systems, volume 65 of Math. Sci. Res. Inst. Publ., pages 93-146. Cambridge Univ. Press, New York, 2014. 
[9] P. Deift, T. Kriecherbauer, K. T.-R. McLaughlin, S. Venakides, and X. Zhou. Strong asymptotics of orthogonal polynomials with respect to exponential weights. Comm. Pure Appl. Math., 52(12):1491-1552, 1999.

[10] P. Deift and X. Zhou. A steepest descent method for oscillatory Riemann-Hilbert problems. Asymptotics for the MKdV equation. Ann. of Math. (2), 137(2):295-368, 1993.

[11] NIST Digital Library of Mathematical Functions. http://dlmf.nist.gov/, Release 1.0.21 of 2018-12-15. F. W. J. Olver, A. B. Olde Daalhuis, D. W. Lozier, B. I. Schneider, R. F. Boisvert, C. W. Clark, B. R. Miller and B. V. Saunders, eds.

[12] T. Ehrhardt. A status report on the asymptotic behavior of Toeplitz determinants with Fisher-Hartwig singularities. In Recent advances in operator theory (Groningen, 1998), volume 124 of Oper. Theory Adv. Appl., pages 217-241. Birkhäuser, Basel, 2001.

[13] M. E. Fisher and R. E. Hartwig. Toeplitz determinants: some applications, theorems and conjectures. Adv. Chem. Phys., 15:333-353, 1969.

[14] F. Franchini. An introduction to integrable techniques for one-dimensional quantum systems, volume 940 of Lecture Notes in Physics. Springer, Cham, 2017.

[15] F. Franchini and A. G. Abanov. Asymptotics of Toeplitz determinants and the emptiness formation probability for the XY spin chain. J. Phys. A, 38(23):5069-5095, 2005.

[16] A. Its and I. Krasovsky. Hankel determinant and orthogonal polynomials for the Gaussian weight with a jump. In Integrable systems and random matrices, volume 458 of Contemp. Math., pages 215-247. Amer. Math. Soc., Providence, RI, 2008.

[17] M. Jimbo. Monodromy problem and the boundary condition for some Painlevé equations. Publ. Res. Inst. Math. Sci., 18(3):1137-1161, 1982.

[18] M. Jimbo and T. Miwa. Studies on holonomic quantum fields. XVII. Proc. Japan Acad. Ser. A Math. Sci., 56(9):405-410, 1980.

[19] B.-Q. Jin and V. E. Korepin. Quantum spin chain, Toeplitz determinants and the Fisher-Hartwig conjecture. J. Statist. Phys., 116(1-4):79-95, 2004.

[20] J. P. Keating and F. Mezzadri. Random matrix theory and entanglement in quantum spin chains. Comm. Math. Phys., 252(1-3):543-579, 2004.

[21] E. Lieb, T. Schultz, and D. Mattis. Two soluble models of an antiferromagnetic chain. Ann. Physics, 16:407-466, 1961.

[22] T. Matsubara and H. Matsuda. A lattice model of liquid helium, I. Progress of Theoretical Physics, 16(6):569, 1956.

[23] B. M. McCoy and T. T. Wu. The two-dimensional Ising model. Harvard University Press, Cambridge, MA, 1973.

[24] M. Shiroishi, M. Takahashi, and Y. Nishiyama. Emptiness formation probability for the one-dimensional isotropic XY model. Journal of the Physical Society of Japan, 70(12):3535-3543, 2001.

[25] B. Simon. Orthogonal polynomials on the unit circle. Part 1, volume 54 of American Mathematical Society Colloquium Publications. American Mathematical Society, Providence, RI, 2005. Classical theory.

[26] G. Szegő. Orthogonal polynomials. American Mathematical Society, Providence, R.I., fourth edition, 1975. American Mathematical Society, Colloquium Publications, Vol. XXIII.

[27] G. Vidal, J. I. Latorre, E. Rico, and A. Kitaev. Entanglement in quantum critical phenomena. Phys. Rev. Lett., 90:227902, Jun 2003.

[28] J. A. Virtanen. Double-scaling limits of Toeplitz determinants and Fisher-Hartwig singularities. In The diversity and beauty of applied operator theory, volume 268 of Oper. Theory Adv. Appl., pages 495-504. Birkhäuser/Springer, Cham, 2018.

Department of Mathematics and Statistics, University of Reading, England

E-mail address: katarzyna.kozlowska@reading.ac.uk

E-mail address: j.a.virtanen@reading.ac.uk 